\title{
Clinical vision and molecular loss: integrating visual psychophysics with molecular genetics reveals key details of normal and abnormal visual processing
}

\author{
Andrew Stockman ${ }^{\mathrm{a}}$, G. Bruce Henning ${ }^{\mathrm{a}}$ and Andrew T. Rider ${ }^{\mathrm{a}}$ \\ ${ }^{a}$ UCL Institute of Ophthalmology, 11-43 Bath Street, London EC1V 9EL, England
}

\section{ABSTRACT}

Over the past two decades we have developed techniques and models to investigate the ways in which known molecular defects affect visual performance. Because molecular defects in retinal signalling invariably alter the speed of visual processing, our strategy has been to measure the resulting changes in flicker sensitivity. Flicker measurements provide not only straightforward clinical assessments of visual performance but also reveal fundamental details about the functioning of both abnormal and normal visual systems. Here, we bring together our past measurements of patients with pathogenic variants in the GNAT2, RGS9, GUCA1A, RPE65, OPA1, KCNV2 and NR2E3 genes and analyse the results using a standard model of visual processing. The model treats flicker sensitivity as the result of the actions of a sequence of simple processing steps, one or more of which is altered by the genetic defect. Our analyses show that most defects slow down the visual response directly, but some speed it up. Crucially, however, other steps in the response sequence can make compensatory adjustments to offset the abnormality. For example, if the abnormal step slows down the visual response, another step is likely to speed up or attenuate the response to rebalance system performance. Such compensatory adjustments are probably made by steps in the sequence that usually adapt to changing light levels. Our techniques and modelling also allow us to tease apart stationary and progressive effects, and the localised molecular losses help us to unravel and characterise individual steps in the normal and abnormal processing sequences.

Keywords: Visual psychophysics, flicker sensitivity, temporal processing, clinical vision, linear systems, gene defects, molecular loss, light adaptation, GNAT2, RGS9, RPE65, GUCA1A, OPA1, NR2E3, KCNV2. 


\section{Contents}

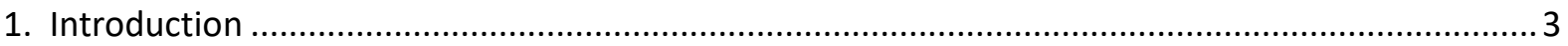

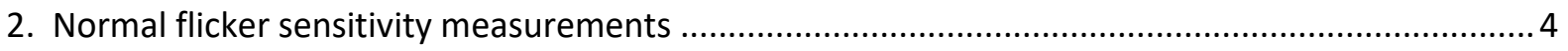

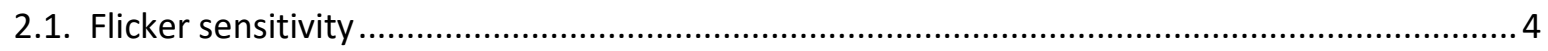

2.2. Flicker sensitivity, light level and light adaptation .......................................................... 7

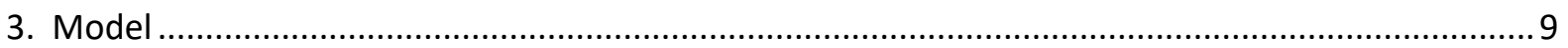

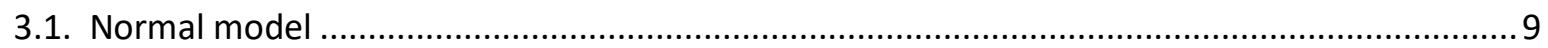

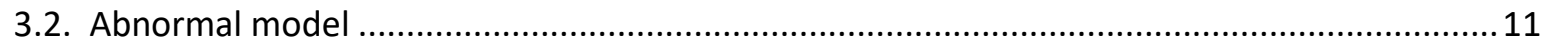

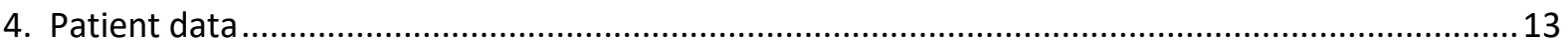

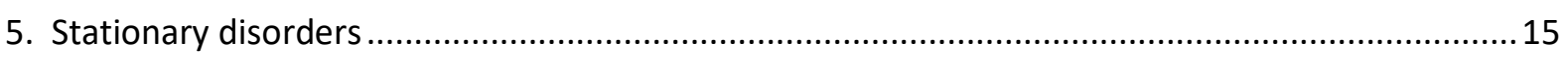

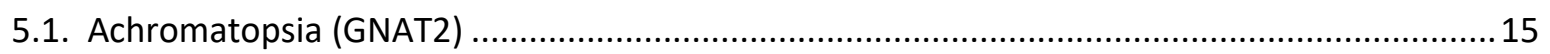

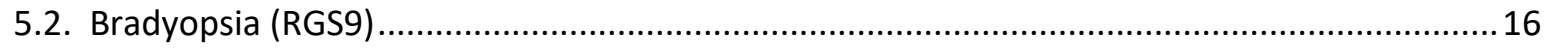

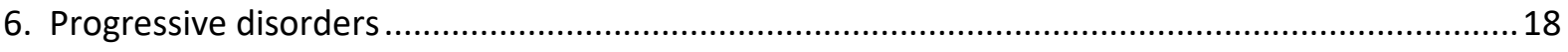

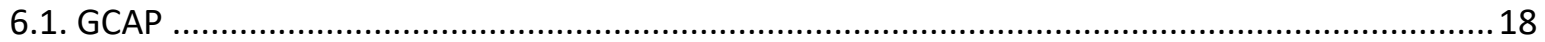

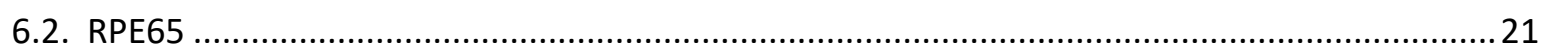

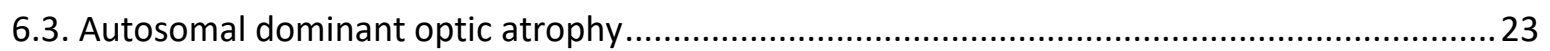

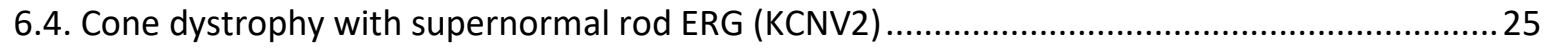

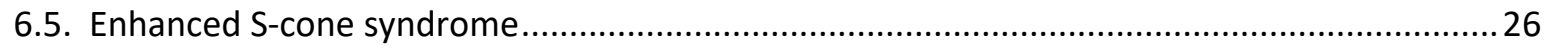

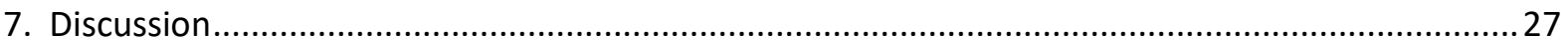

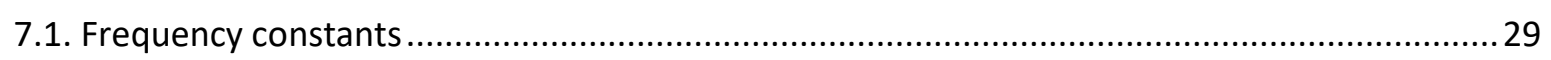

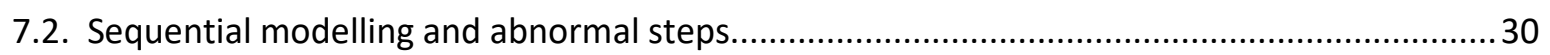

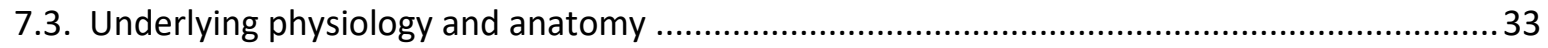

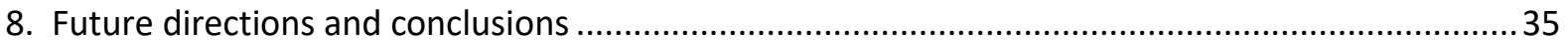

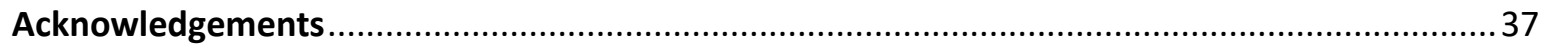

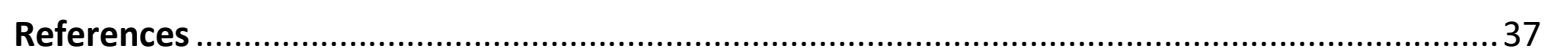

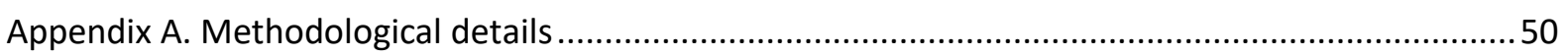

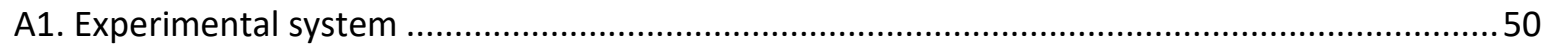

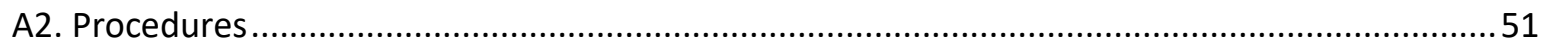

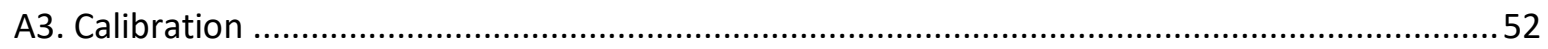

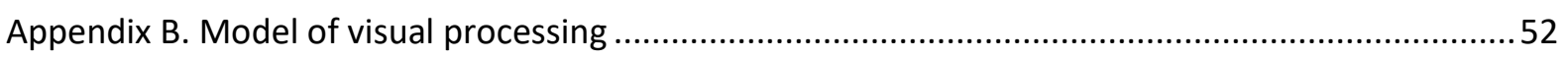

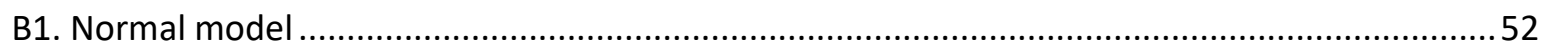

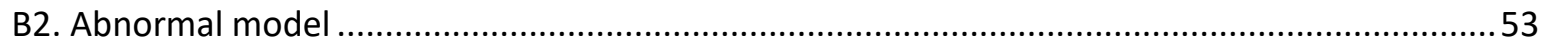

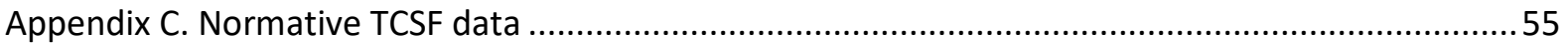

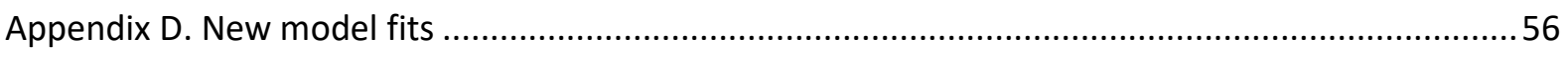

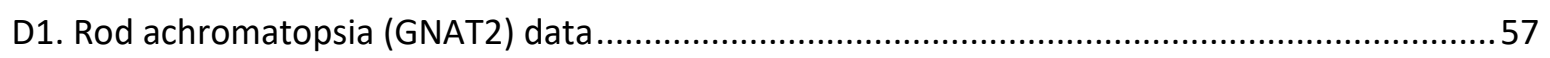

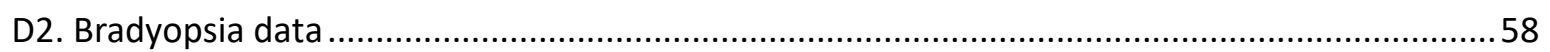

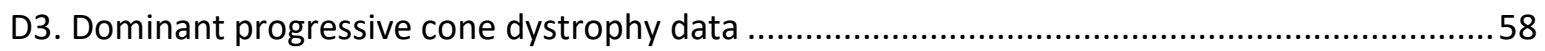

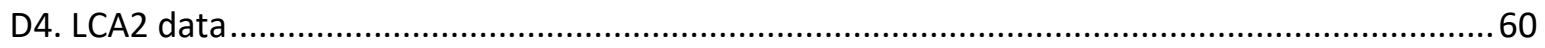


D5. DOA data. .60

D6. Supernormal rod ERG data. 61

\section{Introduction}

Careful measurements of visual performance can reveal important details about the inner working of the human visual system. Such behavioural measurements are broadly known as "psychophysical" measurements, and the area of research as "visual psychophysics". Relating the results of psychophysical experiments to the underlying physiology and anatomy of the eye and brain is always challenging but relating them to specific biochemical and neural processes at the molecular level is even more challenging. A start has been made thanks to the co-operation of patients with visual disorders caused by pathogenic genetic variants that result in specific molecular losses that affect the visual pathways in various ways. These visual disorders typically change the processing speed of one or more stages in the visual processing stream, and such changes can be assessed and characterised by traditional psychophysical measurements of sensitivity to flickering light.

Here, we take a fresh look at clinical measurements of flicker sensitivity made in our laboratory at the UCL Institute of Ophthalmology over the past 20 years. By analysing the data using a sequential model of visual processing (Rider et al., 2019), the results can be used to tease apart the properties of normal and abnormal processing stages in the visual pathway. Additionally, as we illustrate below, the measurements can provide straightforward clinical assessments useful for diagnosis, monitoring disease progression, and testing novel therapeutic interventions. To help quantify performance, we propose a simple new measure of flicker sensitivity that we refer to as the "frequency constant".

[Insert Figure 1 about here]

Our work has focussed on patients with inherited retinal disorders. Figure 1 shows on the left a much-simplified schematic representation of the retina and on the upper right a section of photoreceptor outer segment. The two schemata illustrate some of the crucial components of the visual cascade (see Stockman et al. 2007b). The retinal locations affected by the genetic variants in the seven inherited retinal disorders in this study are indicated by the red 
circles. The locations lie either in the cascade of reactions that transform the absorption of a photon into a neural signal, or in the postreceptoral retinal circuitry. The disorders we have investigated (with the affected genes in parentheses) include: $(\mathbf{A})$ rod monochromacy (GNAT2), (B) Bradyopsia (RGS9), (C) autosomal dominant cone dystrophy (GUCA1A), (D) Leber's congenital amaurosis, LCA2 (RPE65), (E) autosomal dominant optic atrophy (OPA1), (F) cone dystrophy with supernormal rod electroretinogram (KCNV2), and (G) enhanced S-cone syndrome (NR2E3). More details about each disorder are given below.

The ability to link molecular genetics to changes in visual performance provides insight into the functioning of both normal and abnormal human vision. Each genetic change causes changes in temporal sensitivity that can be linked to the molecular deficit itself; many disorders typically slow down the visual response, leading to a loss in sensitivity to rapid flicker relative to sensitivity to slow flicker and steady lights. However, our model also indicates that compensatory adjustments are typically made by other processes in the sequence that act to offset the adverse effects of the slowed response by speeding it up and/or attenuating it. These compensatory processes are likely to be linked to the normal mechanisms of light adaptation, which regulate sensitivity by changing the speed of response and/or by altering the overall gain (see below).

We begin by describing our method of measuring changes in the speed of visual processing. We then introduce the sequential model of visual processing that we use to interpret normal and abnormal data. Our techniques and modelling provide a new way of integrating clinical psychophysics and molecular genetics. The aim in this review is to provide enough information in the main text and the Appendices to allow others to use our techniques, and easily reproduce our measuring equipment. New normative data for future comparisons are tabulated in Appendix C.

\section{Normal flicker sensitivity measurements}

\subsection{Flicker sensitivity}

We employ the classical psychophysical method of measuring sensitivity to a flickering light as a function of temporal frequency (de Lange, 1952; Ives, 1922). As illustrated in Figure $2 \mathrm{C}$, the observer sees a patch of flickering light and adjusts the amplitude of the flicker to find, 
at a fixed frequency and constant mean intensity, the amplitude at which the flicker disappears. This is the amplitude "threshold". The reciprocal of the amplitude threshold gives the observer's amplitude sensitivity at a given frequency of flicker-the lower the threshold, the higher the sensitivity. Varying the amplitude (defined as the maximum excursion of the sinusoidally flickering waveform away from the mean level) while keeping the time-averaged level constant is equivalent to varying the "contrast" of the flicker (defined, for sinusoidally flickering light as the ratio of the amplitude to the mean level).

The sinusoidally flickering target used in our experiments was a circular, foveally-centered, $4^{\circ}$ diameter, red (650-nm) disc superimposed on larger steady circular blue (481-nm) disc; this combination of colours ensures that the flicker was detected by cones rather than rods and mainly by long-wavelength sensitive ( $L$ ) cones rather than by middle-wavelength sensitive (M) or short-wavelength sensitive (S) cones (see Figure A1 in Appendix A where methodological details are provided).

Figure 2A shows flicker sensitivities for 17 normal observers (mean age, 35 years; range 21-65 years) obtained at the standard light level (which we call the High level, see Appendix A). These are the data for normal observers that we will later compare with patient data also measured at the High level. The mean values are provided in Table C1 of the Appendix. This intensity level (3.25 $\log _{10}$ photopic trolands) is useful for examining everyday visual function, as it corresponds approximately to the brighter light levels typically encountered during daily life, e.g., under bright indoor illumination or viewing a high-brightness computer monitor or TV screen of about 250 to $300 \mathrm{~cd} / \mathrm{m}^{2}$.

[Insert Figure 2 about here]

The horizontal axes in Figures $2 \mathrm{~A}$ and $\mathrm{B}$ are flicker frequency in $\mathrm{Hz}$ and the vertical axes are the logarithm of amplitude sensitivity for seeing the flicker. The flicker sensitivity functions for observers with normal vision all have a similar shape: at low frequencies, sensitivity increases until it reaches a maximum near $10 \mathrm{~Hz}$, and then, on these co-ordinates, decreases linearly with frequency, as shown by the straight grey lines that have been fitted to each observer's data. This indicates that fall-off of amplitude sensitivity with increasing frequency is exponential above about $10 \mathrm{~Hz}$ (see also Kulikowski, 1971; Rider et al., 2019; Watson and Ahumada, 2015).The average of the individual data sets is shown in Figure 2B (by the orange 
line). Further, the individual flicker sensitivities shown in 2B have been shifted vertically to line up above $15 \mathrm{~Hz}$ with the mean function. Different vertical shifts represent differences in the overall sensitivities of the observers, and it can be seen that much of the individual variability can be accounted for by differences in overall sensitivity.

The linear relation between log sensitivity and frequency is indicated by the straight brown line in Figure 2B line fitted at frequencies above $10 \mathrm{~Hz}$. The line indicates that the decline of sensitivity with frequency over this range is exponential and thus it can be summarized easily as the change in frequency required to reduce sensitivity by some constant factor. We choose a constant factor of 10 (corresponding to $1 \log _{10}$ unit in the vertical axis of all our sensitivity plots) and will refer to the required change in frequency as the frequency constant by analogy with the concept of a time constant in physics and engineering. The time constant is the change in time required to reduce the temporal response by a specific factor, typically a factor of $\mathrm{e}^{1} \approx 2.7$ (sometimes a factor of 2 is used instead to emphasise the half-life). The frequency constant in the frequency domain is therefore a concept similar to the time constant in the time domain: in one case it is the change in frequency required to reduce the frequency response by a specific factor-by 10 in our case, and in the other, it is the change in time required for the temporal response to fall by a given factor. The frequency constant for the mean normal observer ( \pm the standard error of the estimate) is $19.19 \pm 0.42 \mathrm{~Hz}$ at the High level: thus, there is a 10 -fold loss of sensitivity for every $19.19 \mathrm{~Hz}$ increase in frequency. An increase in the visual response at high frequencies relative to low frequencies results in a higher frequency constant and is produced by faster visual processing. Slower visual processing decreases the response at higher frequencies relative to low frequencies and thus results in a lower frequency constant. Consequently, higher frequency constants imply faster visual processing, and lower frequency constants imply slower processing (see below).

The frequency constants estimated individually for the 17 normal observers show no correlation between the frequency constant and age, suggesting the visual system does not naturally slow with age (Spearman correlation coefficient $-0.27 ; P=0.30$ ).

We describe the flicker sensitivity data for patients below. In general, patients are less sensitive than normal observers, particularly at higher frequencies. Crucially, however, the decline of sensitivity with frequency for patients-like that for normal observers-is exponential but with characteristically different frequency constants. As the frequency 
constant can be determined from as few as two points, it has the potential to be a useful and efficient metric for temporal visual performance in clinical settings.

\subsection{Flicker sensitivity, light level and light adaptation}

The human visual system works well over brightness levels that can vary by as much as $10^{12}$ even though the neurons in the visual pathway have response ranges of only 1000 -fold or less (e.g., Barlow and Levick, 1976; Shapley and Enroth-Cugell, 1984). Vision works well over this range in part by sharing the range between rod and cone photoreceptors (Parinaud, 1881; Schultze, 1866). However, the key control process for cone-mediated vision is that the conedriven response speeds up as the mean light level increases (Bills, 1920; Ferry, 1892; Porter, 1902; Pulfrich, 1922; Rogers and Anstis, 1972; Stockman et al., 2006). As light level increases the signal threatens to overwhelm the visual system by exceeding the neurons' useful operating range. Speeding up is a form of light adaptation that reduces sensitivity to low frequencies and steady mean light level, thus keeping the system within its useful operating range. Higher frequencies are relatively unaffected by the speeding up. We are able to see faster flicker in bright conditions mainly because the amplitude of the flicker can be increased. Consequently, with light adaptation the visual system becomes relatively more sensitive to higher-frequency flicker, a change that can be characterised and quantified by measuring changes in flicker sensitivity. Other mechanisms important for light adaptation are changes in sensitivity that are independent of frequency (sometimes called gain-control or attenuation), which has been likened to putting on dark glasses (Geisler, 1978; MacLeod, 1978). Gaincontrol mechanisms seem to operate predominantly at higher light levels and are a result of neural factors and photochemical factors discussed below (Rider et al., 2019). As we develop below, these light adaptation mechanisms may also play an important compensatory rôle in adjusting sensitivity in patients with visual defects.

The flicker-sensitivity functions of Figure 2 characterise temporal performance at a single average light level; to investigate light adaptation we must measure the functions at various light levels. In our clinical experiments, we have used four light levels, which we refer to as Low, Medium-Low, Medium-High and High. Figure 3 shows mean flicker sensitivity curves averaged over 8 normal observers (mean age, 46 years; range 26-65 years) obtained at the 
High (yellow circles), Medium-High (orange triangles), Medium-Low (red squares) and Low (brown inverted triangles) intensity levels. The vertical axis is again the logarithm of amplitude sensitivity for seeing the flicker and the horizontal axis is frequency $(\mathrm{Hz})$. The data are the mean performance of eight observers and the error bars show \pm 1 S.E.M. These are the data for normal observers that we compare with patient measurements in which the adaptation level was varied. The mean values are provided in Table C2 of the Appendix.

[Insert Figure 3 about here]

The flicker-sensitivity curves in Figure 3 change shape with light level in ways that are characteristic of normal light adaptation. At the Low level (inverted brown triangles), flicker sensitivity falls monotonically with increasing frequency; at higher levels, the flicker sensitivities are peaked so that sensitivity falls at frequencies both below and above the peak; the peak frequency increases as light intensity increases and also the maximum visible flicker increases.

As in Figure 2 , when plotted on these semi-logarithmic coordinates the high-frequency data follow straight lines at each brightness level although the slope is steeper at the lower levels, meaning that observers become relatively less sensitive to fast flicker in dim conditions (e.g., Rider et al., 2019). The frequency constants of the lines \pm the standard error of the estimate at the Low (brown line) and Medium-Low (red line) levels, which are $13.90 \pm 0.68$ and $13.60 \pm 0.59 \mathrm{~Hz}$, respectively, are roughly the same, but the frequency constants increase to $16.48 \pm 0.55 \mathrm{~Hz}$ (orange line) at the Medium-High level and to $19.80 \pm 0.57 \mathrm{~Hz}$ (yellow line) at the High level. The frequency constants allow us to monitor how light adaptation changes the speed of the visual response.

The mean age of the 17 normal observers whose data are shown in Figure 2 was 35 years, while the mean age of the subset of 8 observers in Figure 3 was 46, yet the frequency constant for the high level changes from only $19.19 \pm 0.42 \mathrm{~Hz}$ to $19.80 \pm 0.57 \mathrm{~Hz}$ between the two groups. This supports our contention above that the frequency constant is independent of age. 


\section{Model}

While the frequency constants provide a quick and efficient way of summarizing visual performance in normal observers and, as we show below, in patients, it is a measure of overall speed that ignores potentially important differences in sensitivity at lower temporal frequencies. Flicker-sensitivity data analyzed at both low and high frequencies can reveal more extensive information about normal and abnormal visual processing. To extract that information, however, we need to apply a model of visual processing. Fortunately, relatively straightforward and plausible sequential models can be used to relate the dynamics of visual processing to flicker sensitivities (e.g., Rider et al., 2019). Before considering its application to patient data, we introduce the model as it applies to, and was developed to explain, normal data.

\subsection{Normal model}

The early visual system can be thought of as a sequence of neural, electro-chemical reactions and connections. We, like many others before us (see review by Shapley, 2009), model the sequence of stages in the early visual system as a series of "leaky integrators" or filters, which we refer to as low-pass (LP) stages. LP-stages are good models of first order chemical reactions and of simple electrical circuits; they have a long history in modelling visual temporal responses; and they are mathematically tractable (e.g., de Lange, 1958; Watson, 1986). An LP-stage is characterised by two parameters, one sets the overall response level and the other controls the speed. We give the speed in terms of the corner frequency, $f_{c}$ in $\mathrm{Hz}$, which is the frequency above which an LP-stage begins to reduce the amplitude of its output significantly. (Next, we describe how comparing normal and clinical data, using our model as a framework, can help to tease apart important processes in the early visual system.) The essential components of our model are shown in Figure 4.

\section{[Insert Figure 4 about here]}

The black line down the centre of the figure represents a sequence of visual processes. The sequence consists of 6 low-pass (LP) stages labelled [1]-[6] , a gain control, $g$, at the beginning of the sequence to control overall signal strength, and 2 feedforward stages, each of which is made up of an LP-stage $[\mathrm{A}]$ or [B], a gain control, $k$, and a signal inversion (-). The 
feedforward stages provide inhibition and the strength of each feedforward inhibition is controlled by $k$. We take the amplitude of the signal as it passes through the model to represent the information passing through the visual system-in analogue form in the receptor and outer retina, but as trains of impulses after the inner retina. Because the effects of the model's components on signal amplitude multiply in the model (see Appendix B), their order in the processing sequence shown in Figure 4 is arbitrary (we discuss a physiologically plausible sequence in the Discussion).

The four panels down the left side of the figure represent probes that show the temporal response of the system at various stages. The temporal or "time-domain" response offers some insight into the operation of the system but more important, from our point of view, is the frequency or "frequency-domain" response shown by the corresponding probes on the right. The panels down the right side show the frequency response corresponding to each of those stages (the frequency response can be obtained by the Fourier transform of the temporal response). The frequency response shows the amplitude of the response at each frequency. We assume that the frequency response after the final LP-stage (Panel 4) represents the human (system) sensitivity to flicker as a function of frequency. The top pair of probes shows the input to the system that is most effective in revealing the system's response characteristics: it is a very brief flash of light in the time domain and its equivalent, in the frequency domain, the sum of sinusoids of all frequencies all of the same amplitude. In general, adding LP-stages in the direct pathway "smears out" the temporal response, so that after three identical LP-stages (probe 2) the temporal response is smeared out and its peak delayed; and the corresponding frequency response (right-hand panel) shows an increasing loss of amplitude with increasing frequency. The high-frequency loss steepens with additional LP-stages and steepening the high-frequency loss reduces the "frequency constant".

Adding feedforward stages sharpens the initial positive response in the time domain (probe 3), advancing its peak, and adding a delayed negative response (compare the response at probes 2 and 3 on the left). The frequency response becomes bandpass - with losses below the maximum as well as above it-and the high-frequency losses become shallower, so increasing the frequency constant (compare the response at probes 2 and 3 on the right). 
Probe 4 illustrates the final response of the system (its output or system response) and it is this signal in the model that we relate to the observers' response to flickering light.

As discussed in Rider, Henning \& Stockman (2019), our model owes much to previous models of light adaptation, many of which incorporate, in different configurations, the elements used in our sequential model (Barten, 1999; de Lange, 1952, 1958, 1961; Ives, 1922; Kelly, 1961; Matin, 1968; Purpura et al., 1990; Roufs, 1972b; Rovamo et al., 1999; Sperling and Sondhi, 1968; Tranchina et al., 1984; Watson, 1986). The model we use was based on fits to normal flicker sensitivity data extracted from seven earlier publications (de Lange, 1958; Kelly, 1961; Roufs, 1972a; Rovamo et al., 1999; Stockman et al., 2006; Swanson et al., 1987; von Wiegand et al., 1995). An important distinguishing feature of our model is that the fall-off in high-frequency sensitivity over the range of frequencies over which flicker is visible follows an exponential course, consistent with the early flicker sensitivity data and with the flicker sensitivity data for normal observers and patients shown here. Thus, the model and data support the concept of a frequency constant.

A well-known feature of sequential LP-stage models, such as ours, is that the response tends toward a power-law at high frequencies, with a negative exponent equal to the number of stages, and so should appear as a straight line in double-logarithmic coordinates, rather than in the semi-logarithmic coordinates we use here. However, we have shown that over the visible range of frequencies an exponential function is a better approximation, while the power-law region is presumed to lie at frequencies too high to be resolved by our visual system, and therefore unamenable to psychophysical measurement (Rider et al., 2019).

\subsection{Abnormal model}

We use the normal sequential processing model of Figure 4 to account for abnormal vision by assuming that the inherited retinal disorders alter one or more of the normal processing stages, thus changing the system response from the affected stage onward, and eventually changing the measured flicker sensitivities.

\section{[Insert Figure 5 about here]}

An example of this approach is illustrated in Figure 5: the normal sequence is shown in the column on the left, an abnormal sequence in the column on the right and the differences in the centre column. The probes show responses in the frequency domain. In 
Figure 5, a genetic variant has caused the second LP-stage to slow down and has thus increased sensitivity to low frequencies and steady lights (compare the frequency responses in the red and green boxes - the difference is shown in the yellow box labelled (A)). If this change simply propagated through the rest of the visual system, the measured differences between normal and abnormal flicker sensitivities for the whole system would be as in the yellow box. By then applying the model to analyse the differences between normal and abnormal flicker, we should be able to estimate the normal and abnormal corner frequencies for the affected stage.

Examples of other single-stage differences on the whole system that we might expect to find are shown inside the cyan box at the bottom of Figure 5. Panel (A) shows, as in the illustration, the effect of a stage slowing down. Panel (B) shows the effect of a stage speeding up that causes a decrease in the low-frequency response relative to the high. Panel (C) shows the effect of a gain control that decreases the response uniformly across all frequencies, and Panel (D) shows the effect of a loss of feedforward inhibition.

Below, we measure and model the sensitivity differences between normal observers and each patient. In general, we find that the differences in the measured flicker sensitivities are not consistent with single stage differences, suggesting that the changes caused by a given defect may not simply propagate through the system. Instead, the modelling requires at least two and in many cases three changes to the standard model to fit the data. We suggest the altered output of an affected stage can cause compensatory adjustments in other otherwise normal stages in the sequence. These compensatory adjustments act to rebalance the system and offset the effects of the abnormality.

For example, if, as in Figure 5, the abnormal stage is one that slows down, its effect will be to increase the response of the system to low temporal frequencies and steady lights. If left unchecked, this increase in response could drive the system beyond the operating range of downstream neurons and lead to the loss of the signal. To remedy the situation, other stages must reduce the gain across all frequencies, as in Panel (C), and/or speed up, as in Panel (B).

In Figure 5, the effects of the original defect, a compensatory adjustment to the overall gain and a speeding up of LP-stages after the stage 2 combine to give the system differences shown in the lower orange panel in Figure 5 . These are the sensitivity differences that we 
measure. In the example shown in Figure 5, the slowing down and a compensatory reduction in gain and speeding up of the response produce this characteristically asymmetrical U-shaped function, which we find for many patients.

Figure 5 shows an example of a defect that slows down the response $(A)$, but it is important to note that compensatory adjustments should also be expected if the defect speeds up (B) or attenuates (C) the response, as we discuss below.

\section{Patient data}

We now turn to flicker sensitivity measurements made in our laboratory in different patient groups, each of which has a genetic defect that affects important molecular or neural pathways in the retina. The locations of the affected molecules were shown in Figure 1 and identified by the letters used in the subheadings below. In each case, we have modelled the flicker sensitivity differences between the patients and normal observers using the model described in the previous section. Patient availability for these studies was limited as the disorders we investigated are rare. The numbers of patients identified by the Genetics Service of Moorfields Eye Hospital with the relevant gene mutations from their cohort of 4241 molecularly diagnosed individuals are 1 (GNAT2), 1 (RGS9), 13 (GUCA1A), 25 (KCNV2), 31 (NR2E3), 51 (RPE65), and 84 (OPA1) (Pontikos et al., 2020). Of these, only a proportion were available to undergo the time-consuming psychophysical tests needed to be carried out for this work in our London laboratories.

Each gene defect and its associated disease are introduced in the next two sections. Further references and details can be found in our original publications. We have grouped the patients according to the affected gene and its associated disease, but it should be noted that the gene mutation can vary between patients, and that this might affect the severity of the disease. Details of the mutations for the Leber's congenital amaurosis (RPE65) patients can be found in Table 1 of Ripamonti et al. (2014b), for the autosomal dominant optic atrophy (OPA1) patients in Table 2 of Majander et al. (2017) and for the cone dystrophy with supernormal rod ERG (KCNV2) patients in Table 1 of Stockman et al. (2014a). We have too few examples of each mutation to draw any useful conclusions about the type of gene mutation and disease severity. 
Patient ages are given in the text and are also noted in the figure keys. Information about other clinical tests undertaken by the patients can be found in the original publications. To facilitate this, the same patient code has been used in this paper as in the original publications. Comparisons between our results and model, and more conventional functional tests, such as full-field stimulus threshold (FST) (Roman et al., 2007) or two-colour automated perimetry (Jacobson et al., 1986; Simunovic et al., 2016), are of limited utility, because such tests use fixed-duration flashes to probe sensitivity rather than flicker of different frequencies. Consequently, unlike flicker measurements, they cannot be used to dissociate changes in the speed of processing (and thus changes in integration time) from changes in overall sensitivity.

A review of the molecular basis of many retinal diseases can be found in Berger, Kloeckener-Gruissem \& Neidhardt (2010). Details of the phototransduction cascade can be found in several review articles (Arshavsky et al., 2002; Burns and Baylor, 2001; Fain et al., 2001; Perlman and Normann, 1998; Pugh and Lamb, 2000; Pugh et al., 1999).

When linking the molecular defects to visual performance it is also important to distinguish between stationary and progressive disorders. In stationary disorders (and probably in the early stages of progressive disorders), the visual losses are likely to reflect the molecular losses caused by the genetic defect more directly. In progressive disorders, however, other disease processes are likely to play a role. Thus, in stationary disorders we may be able to link the visual losses more firmly to the molecular losses. In advanced progressive disorders, the visual losses may reflect mainly the deterioration and/or loss of retinal components and circuitry, such as photoreceptors or ganglion cells. In such cases, the results may also reflect altered or abnormal circuitry. By examining data from patients in different stages of a progressive disease it may be possible to discriminate features reflecting the effects of the molecular loss, from features that may reflect the progressive nature of the disease.

The original data from our earlier work have been reanalysed for this review using the model described above, details of which are provided in the Appendices. Further details of the molecular genetics and genetic variants can be found in the original publications for each patient using the same patent identifiers used here. 


\section{Stationary disorders}

\subsection{Achromatopsia (GNAT2)}

The first results we describe are from two patients, a father (aged 48) and son (24), with a defect in GNAT2 that encodes the $\alpha$-subunit of the cone G-protein transducin. Transducin (see A Figure 1) is a key component in the cone transduction cascade: $\mathrm{G} \alpha$ binds to and activates the phosphodiesterase effector molecule (PDE6 in Figure 1). Activated PDE6 then catalyses the hydrolysis of cyclic guanosine monophosphate leading to the closure of the cyclic-nucleotide-gated ion channels in the receptor plasma membrane and the membrane hyperpolarization that constitutes the receptors' signal-the "photoresponse". Without cone $\alpha$-transducin, patients should be rod achromats with no cone vision. Surprisingly, however, as shown in the next figure, we found both patients had residual cone vision at intensities high enough to bleach a significant amount of cone photopigment even though the rod mediated visual pathways were saturated and did not contribute to flicker sensitivity (Stockman et al., 2007b). (That these measurements were cone- and not rod-mediated was confirmed by spectral sensitivity measurements.)

[Insert Figure 6 about here]

Figure $6 \mathrm{~A}$ shows the mean of three flicker sensitivity determinations measured at the High level for the father (red triangles, aged 48) and son (green inverted triangles, aged 24) replotted from our original paper (Stockman et al., 2007b). The numbers in the legend here, as in all the figures for patient data, correspond to their age at testing. For comparison, the mean for 17 normal observers over the same frequency range (orange line) is shown together with \pm 2 S.E.M. across the normal observers (dashed white lines). The patients' flicker sensitivities extend to only $6 \mathrm{~Hz}$ and $2 \mathrm{~Hz}$ for the father and son, respectively. Both functions are low pass in shape and have steep slopes with frequency constants of $7.10 \mathrm{~Hz}$ (red line) and $6.40 \mathrm{~Hz}$ (green line), much lower than the frequency constant of $19.19 \pm 0.42 \mathrm{~Hz}$ for normal observers.

The finding that cone vision in the absence of $\alpha$-transducin occurs only at intensity levels that bleach a significant amount of cone photopigment suggests that cone vision is sustained by a photobleaching product that secondarily activates the cone transduction 
cascade (Stockman et al., 2007b). Such secondary activation is thought to be the origin of cone "background equivalence" (e.g., Pianta and Kalloniatis, 2000; Stiles and Crawford, 1932), the long-established observation that the after-effects of photopigment bleaches are comparable to the effects of real background lights (for reviews, see Fain et al., 2001; Lamb and Pugh, 2004; Leibrock et al., 1998).

We can learn more about the properties of this hypothetical bleaching photoproduct by applying our model to the sensitivity losses. Figure 6B shows the losses for the father (red triangles) and son (green inverted triangles) relative to normal sensitivity. As in other graphs of this type, the logarithms of the sensitivity difference (losses in this case) are plotted as a function of frequency. No difference from normal produces zero logarithmic loss so the increasing losses in Figure 6B are substantial. We can account for the losses using a model that places two low-pass stages into the processing streams of the patients. The model's predictions are shown in Figure 6B as smooth curves through the measured losses. For the father, the two interpolated stages both have corner frequencies of $1.01 \mathrm{~Hz}$ (red line) and for the son they have corner frequencies of $1.07 \mathrm{~Hz}$ (green line). See Appendix D1 for further details.

The losses, then, are consistent with the light-induced changes in the lifetime of a hypothetical photoproduct that depends on two low-pass stages; the two stages can be likened to two simple, first-order reactions. In terms of likely mechanism, the abnormal stages have somehow bypassed the blockage in the cascade caused by the lack of $\alpha$-transducin in the abnormal retinae and thus partially restored the processing sequence.

In summary, the losses for both patients can be understood resulting from processes (A) (slowing) and (C) (overall sensitivity loss) that were shown at the bottom of Figure 5.

\subsection{Bradyopsia (RGS9)}

The second example of a stationary disorder is a single patient (aged 62) with a defect in RGS9-1, a gene that encodes a GTPase accelerating protein in the transduction cascade (see $\mathbf{B}$ in Figure 1). This protein increases the rate of deactivation of the $\alpha$-transducin-PDE6 complex after its activation by light and thus helps to speed up the offset of the light response. Its loss results in a disease known as Bradyopsia or "slow vision" (Nishiguchi et al., 
2004). Bradyopsia is a stationary disease with early childhood onset, mild photophobia, difficulty tracking moving objects, moderate reductions in spatial acuity, and ERG abnormalities; but with normal colour vision and normally appearing fundi (Cheng et al., 2007; Hartong et al., 2007; Michaelides et al., 2010; Nishiguchi et al., 2004).

[Insert Figure 7 about here]

To investigate how visual processing in this patient depends on light intensity, flicker sensitivities were measured at the same four light levels as for normal observers-the Low, Medium-Low, Medium-High and High levels of the 650-nm stimulus. The flicker sensitivities \pm 1 S.E.M. for the four levels are plotted in Figure 7A as brown, red, orange and yellow triangles, respectively. For comparison, the mean normal observer data from Figure 3 are shown as coloured circles. Apart from the very lowest frequencies, the four functions for the patient all follow straight lines above about $2 \mathrm{~Hz}$, with frequency constants of $15.59 \pm 1.16$ (brown line), $18.31 \pm 0.58$ (red line), $16.77 \pm 0.39$ (orange line) and 27.14 \pm 1.67 (yellow line) $\mathrm{Hz}$. These frequency constants are greater than the normal constants of $13.90 \pm 0.68,13.60$ $\pm 0.59,16.48 \pm 0.55$ and $19.80 \pm 0.57$ at the corresponding levels, respectively( dashed white lines); and there is a much greater difference between the two lower levels in the patients than in the normal observers. Thus, the frequency constants are substantially higher at three of the four levels indicating that vision in the patient-although less sensitive in absolute terms - is relatively faster than normal despite the expectation from the genetic characteristic that it should be slower. How can this be explained?

Figure 7B shows the sensitivity losses (coloured symbols) for the patient relative to the normal data. At lower frequencies and lower mean radiances (brown and red triangles), the sensitivity losses increase with frequency; that is consistent with slowing down of the visual response. Yet, at higher frequencies the sensitivity losses are substantially reduced as frequency increases; that, on the other hand, is consistent with speeding up of the visual response.

Slowing down the visual response is the expected direct consequence of the loss of the RGS9 molecule in Bradyopsia. However, to account for the asymmetrical U-shaped pattern of the losses, an additional change is required: a speeding up of the response at a different stage possessed by both patients and normal observers. This speeding up, although 
perhaps unexpected, is nonetheless easily understood as an indirect consequence of the RGS9 loss. By slowing down the response, the loss of RGS9 increases the time-averaged (steady) signal downstream from the position of the affected stage. To later stages, this is comparable to being exposed to a brighter steady light. Consequently, later stages capable of sensitivity regulation will act to offset this increase in an attempt to restore the operating points within their normal range of operation; this can be achieved either by speeding up their response or by attenuating it, which cause the distinct patterns of loss shown in Figure 5 [compare examples (B) and (C)]. Thus, our model accounts for the data (lines in Figure 7B) by slowing one LP-stage and speeding a different one. We conclude that Bradyopsia is an example of an abnormal stage slowing down the visual response, which causes a separate stage to speed up to maintain sensitivity at low frequencies (thus, examples (A) and (B) in Figure 5). However, in this case the speeding up appears to have overcompensated for the slowing down, since the factor by which the affected stage slows down is less than factor by which the compensating stage speeds up. We discuss a possible explanation in the Discussion.

\section{Progressive disorders}

\subsection{GCAP}

The first example of a progressive disorder comes from measurements made in a group of four individuals from the same family, all of whom suffer from a dominant, progressive cone dystrophy caused by a missense mutation in the GUCA1A (GCAP1) gene, a gene that encodes a guanylate cyclase activating protein, which is responsible for the restoration of cGMP following its light-activated hydrolysis by the activated effector molecule PDE6, and thus also active in terminating and shaping the photoresponse (Payne et al., 1998) (see C Figure 1). The defect causes retinal guanylate cyclase (GUCY2D) to be activated at low light levels, thus inappropriately speeding up the visual response. Consequently, we should expect data from these patients to show evidence for a speeding up of the visual response but probably complicated by the progressive losses. The mutation is referred to as gain-offunction mutation, since it enhances the activity of the affected protein. For further details, see Stockman et al. (2014b). The resulting disease, autosomal dominant cone dystrophy, is 
associated with mild photophobia, reduced central vision, and reduced colour vision, and is accompanied by a gradual deterioration of visual acuity and colour vision with age (Downes et al., 2001; Michaelides et al., 2006; Michaelides et al., 2005b; Payne et al., 1998).

\section{[Insert Figure 8 about here]}

Figure 8A shows, in four separate panels, the flicker sensitivities for four patients, GP1 (triangles, aged 39), GP2 (circles, 41), GP3 (inverted triangles, 51) and GP4 (diamonds, 55) all measured at the Medium-Low (red symbols), Medium-High (orange symbols) and High (yellow symbols) levels. The standard errors for the patients are across three separate flicker sensitivity measurements and across the 17 observers for the normal observers. The lines that best-fit the high frequency ends of the flicker sensitivity functions are shown by the red, orange, and yellow solid lines in each panel. The frequency constants for each patient and for the normal mean are tabulated in Table 1 together with \pm 1 standard error of the fit.

The frequency constants for GP1 are much greater than those for normal observers but change relatively little with light level, which suggests, as expected, a faster visual response and one that does not speed up with light level. The frequency constants for GP2 are comparable to those for normal observers, but, unlike normal observers, GP2's frequency constant decreases with light level, which suggests that the visual response slows down rather than speeds up as light level increases. The frequency constants for GP3 and GP4 become much greater between the first two levels and then decrease again between the second and third levels, consistent with a speeding up of the system at lower levels and a slowing down at higher levels. Table 1 gives the frequency constants.

\section{TABLE 1}

\begin{tabular}{|c|c|c|c|}
\hline \multirow{2}{*}{ Patient } & \multicolumn{3}{|c|}{ Frequency constants $(\mathrm{Hz})$} \\
\cline { 2 - 4 } & Medium-Low & Medium-High & High \\
\hline GP1 & $33.00 \pm 4.06$ & $27.83 \pm 1.84$ & $27.33 \pm 1.25$ \\
\hline GP2 & $18.85 \pm 1.53$ & $13.33 \pm 1.14$ & $14.25 \pm 0.74$ \\
\hline GP3 & $10.41 \pm 2.62$ & $29.74 \pm 1.67$ & $19.68 \pm 0.93$ \\
\hline
\end{tabular}




\begin{tabular}{|c|c|c|c|}
\hline GP4 & $10.59 \pm 1.35$ & $18.14 \pm 1.00$ & $12.12 \pm 0.83$ \\
\hline Normals & $\mathbf{1 3 . 6 0 \pm 0 . 7 4}$ & $\mathbf{1 6 . 4 8} \pm \mathbf{0 . 5 5}$ & $\mathbf{1 9 . 8 0} \pm \mathbf{0 . 5 7}$ \\
\hline
\end{tabular}

Table 1. Frequency constants for patients with dominant, progressive cone dystrophy.

Figure 8B shows the sensitivity losses for GP1 (green triangles), GP2 (purple circles), GP3 (inverted yellow triangles and GP4 (blue diamonds) at the Medium-low (left panel), MediumHigh (middle panel) and High (right panel) levels relative to the normal. A striking feature of the losses is that GP1, and possibly GP2 at the lowest level, show a reduction in sensitivity loss as the frequency increases but that GP3, and GP4, and, at the higher levels, GP2, show an increase in sensitivity loss that gets larger with increasing frequency and with increasing age. A relative speeding up is expected from the removal of GCAP-mediated control and is clearly apparent in the youngest patient (GP1) for whom the loss relative to normal gets smaller as frequency increases above about $8 \mathrm{~Hz}$, particularly at the lower two levels. For the second youngest (GP2) at the Medium-Low level, the losses follow the U-shaped function that indicates one stage slowing down and another speeding up. A plausible supposition is that the effect of the speeding up of the visual response is masked in the older patients' data because their visual systems are overwhelmed by the progressive losses that slow down the visual response. The slowing down also restricts the range of frequencies that can be measured making a determination of a U-shaped function impossible (Stockman et al., 2014b). As discussed above, a slower LP-stage will increase sensitivity at low frequencies, but these patients all have significant losses compared to normal at low frequencies, suggesting the progressive slowing down is accompanied by large reductions in frequency-independent gain, which may reflect cone photoreceptor loss.

We can account for the losses in Figure $8 \mathrm{~B}$ by varying the model in the same way we varied it to account for losses for the Bradyopsia patient: two LP-stages that have the same corner frequency in the normal observers are adjusted in opposite ways in the patients with one speeding up and the second slowing down. The model fits are shown by the solid lines in each graph in each panel of Figure 8B; the details of the fits can be found in Appendix D3. In all patients, one stage speeds up at every level. In GP1, the youngest patient, the second stage has the same corner frequencies as the normal stage. In GP2-GP4, the second stage 
has lower corner frequencies than the normal stage with GP3 and GP4 being more affected than the second youngest patient GP2.

In summary, the losses for these patients are consistent with examples (A), (B), and (C) in Figure 5 (slowing at one stage, speeding at a second stage, and gain control), except for GP1 who shows no evidence for a stage slowing down. The model fits suggest that in the youngest patient the mutation speeds up the visual response as expected, but that in the older patients the progressive nature of the disease slows down the response and reduces the overall gain leading to severe losses of temporal sensitivity. There is no clear evidence in GP1 for a later stage slowing down to compensate for the speeding up of the defective stage. This may simply be because at lower light levels the later stages are at the lower end of their adaptation ranges before the effects of the abnormal stage are considered. Consequently, they cannot slow down enough to compensate for the abnormal speeding up of the abnormal stage. The slowing down in the older patients, GP2-GP4, is assumed to be due to progressive damage.

\subsection{RPE65}

The next example is of five patients suffering from Leber's congenital amaurosis (LCA2), which is caused by mutations in RPE65 (see $\mathbf{D}$ in Figure 1), a gene that encodes an isomerase that is a key component of the visual cycle (e.g., Gu et al., 1997; Marlhens et al., 1997; Perrault et al., 1999b; Redmond et al., 1998). The visual cycle is the biochemical pathway that regenerates the chromophore in the visual pigment, 11-cis retinal, after it has absorbed a photon (e.g., Lamb and Pugh, 2004). The rod photoreceptors have access to 11-cis retinal only via RPE65, so that LCA2 patients are usually night-blind; by contrast, cone photoreceptors also gain 11-cis retinal through a secondary pathway that does not depend on RPE65, thus enabling cone-mediated vision in younger LCA2 patients (Wang and Kefalov, 2011; Wu et al., 2004; Znoiko et al., 2002). Unfortunately, this pathway does not sustain cone vision in the long-term, so that patients become blind in the third or fourth decade of life (Hanein et al., 2004; Perrault et al., 1999a).

[Insert Figure 9 about here] 
The flicker sensitivity data shown in Figure 9 are baseline measurements (Ripamonti et al., 2014b) for patients who were part of a gene therapy trial (Bainbridge et al., 2015; Bainbridge et al., 2008). Measurements were made separately in each eye and are shown averaged across eyes. Only the data for the five older patients (S1, S3, S5, S6, S12), who used our standard testing equipment, are shown. Figure $9 \mathrm{~A}$ shows flicker sensitivities \pm 1 S.E.M. for S1 (green diamonds, aged 24), S3 (blue triangles, 18), S5 (red circles, 23), S6 (purple hexagons, 17) and S12 (blue squares, 19) and the mean normal data \pm 2 S.E.M. (solid orange and dashed white lines) over the patient's restricted frequency range. The flicker sensitivity functions are all slightly bandpass but peak at low frequencies between 1 and 2 $\mathrm{Hz}$ and are restricted to temporal frequencies well below the normal range. Above about 1$2 \mathrm{~Hz}$ the patients' data again fall along straight lines, indicating exponential sensitivity losses with frequency, with best-fitting slopes with frequency constants of $9.75 \pm 0.59$ (S1, green line), $13.63 \pm 0.92$ ( $S 2$, dark blue line), $9.83 \pm 0.35$ (S5, red line), 10.24 \pm 0.73 (S6, purple line) and 16.94 \pm 1.37 (S12, blue line), compared to a normal value of $19.19 \pm 0.42 \mathrm{~Hz}$.

Visual performance gets worse from patients $\mathrm{S} 5 \rightarrow \mathrm{S} 12 \rightarrow \mathrm{S} 6 \rightarrow \mathrm{S} 3 \rightarrow \mathrm{S} 1$. There is no clear age-related pattern to these results, but the age range is limited to seven years. Figure 9B shows the sensitivity losses for each patient (coloured symbols) relative to the normal sensitivities. The losses for each patient have been vertically aligned with the mean patient losses (plotted as dotted yellow squares with their just visible error bars showing \pm 1 S.E.M.). This alignment makes clear that although the losses vary in severity, their frequencydependent form is similar across patients. We can account for the mean losses by having two stages in the normal processing sequence slow down from corner frequencies of $20.79 \mathrm{~Hz}$ in normal observers to $2.29 \mathrm{~Hz}$ in the patients. The prediction is shown by the solid red line in Figure 9B. See Appendix D4 for further details of the fitting.

In summary, the losses for these patients are consistent with examples ( $A$, slowing) and (C, gain control) in Figure 5. The slowing down processing in the RPE65-mutant visual pathway can be linked to changes at the molecular or neural level caused directly or indirectly by the mutation. For example, the loss may be linked to a limiting sluggish molecular process that maintains the residual function within damaged cone photoreceptors, or to indirect pathways that might predominate over direct pathways following photoreceptor loss (Ripamonti et al., 2014b). The progressive nature of the 
disease then manifests as a reduction in the gain, consistent with the gradual loss of functioning cones.

In general, the slowing down of processing at an abnormal stage or stages results in an increase in relative sensitivity to low-frequency and steady signals. These increases must be compensated for by later stages turning down their gain or speeding up their response to keep the system in a useful operating range. In these data, there is little evidence for a compensatory speeding up of later stages, since the sensitivity losses in Figure 9B do not decrease at higher frequencies, except perhaps for S12 (blue squares), but the data are restricted to low frequencies.

\subsection{Autosomal dominant optic atrophy}

The third example of a progressive disorder is from measurements made in 11 patients suffering from autosomal dominant optic atrophy (DOA) caused by a heterozygous mutations in the OPA1 gene, a gene that encodes an inner mitochondrial membrane protein (Alexander et al., 2000; Burte et al., 2015; Delettre et al., 2000). This disease affects retinal ganglion cells with no evidence of functional or structural abnormalities in the preceding neural stages in the outer retina (Cohn et al., 2008; Johnston et al., 1979; Kjer, 1959; Kjer et al., 1983; Votruba et al., 1998; Yagasaki et al., 1986a; Yu-Wai-Man et al., 2010). Patients usually become symptomatic in childhood exhibiting moderate losses in spatial acuity, central field defects, and colour vision defects; moreover, optical coherence tomography studies show a loss of retinal ganglion cells (Barboni et al., 2014; Cohn et al., 2008; Kjer, 1959; Milea et al., 2010; Ronnback et al., 2013; Ronnback et al., 2015; Votruba et al., 1998). The visual losses in DOA should not reflect changes in the photoreceptors, which would be expected to perform normally (see $\mathbf{E}$ in Figure 1).

[Insert Figure 10 about here]

Figure 10A shows the flicker sensitivities measured at the High intensity level for the DOA patients: P1 (red triangles, aged 13) , P2 (dark-blue squares, 24), P3 (green diamonds, 27), P4 (inverted green triangles, 29), P5 (inverted blue triangles, 39), P6 (orange squares, 47), P7 (pink circles, 47), P8 (blue diamonds, 47), P9 (orange triangles, 52), P10 (khaki circles, 54) and P11 (brown diamonds, 25); together with the mean normal data (orange and 
dashed white lines). In general, the patient sensitivity functions are bandpass in shape, like the normal functions, but they peak at about $5 \mathrm{~Hz}$ rather than $10 \mathrm{~Hz}$ for the normal observers, and the loss in sensitivity at frequencies below the peak is less than for normal observers. The high-frequency slopes generally follow straight lines as for other patient types and normal observers. The best-fitting straight lines over the high-frequency region of each patient are shown by the solid and dashed grey lines. The slopes of the best-fitting lines are very similar except for P1 and P3 (dashed grey lines) whose slopes are much shallower indicating higher frequency constants. The frequency constant for the mean patient data is $23.27 \pm 0.83 \mathrm{~Hz}$ (red-black dashed line). This is slightly greater than the frequency constant of $19.19 \pm 0.42 \mathrm{~Hz}$ for normal, suggesting that processing on average is slightly faster in the DOA patients.

Figure 10B shows the sensitivity losses for the patients (coloured symbols) relative to the normal sensitivities. The mean losses \pm 1 S.E.M are shown by the yellow dotted squares and the individual data have been vertically aligned with the mean to illustrate a consistent pattern of losses despite large differences in overall sensitivity. The losses can be accounted for by two LP-stage in the normal observer with the same corner frequencies of $14.71 \mathrm{~Hz}$, one of which slows down to $3.55 \mathrm{~Hz}$ in the patients and the other of which speeds up to the limit of $100 \mathrm{~Hz}$ imposed in the fit (red and black dashed line).

The evidence for a stage speeding up seems clearer in the data for younger patients (under 40 years of age), than in those for older patients. Accordingly, we split the patients into two age groups and fitted the groups separately (Figure 10C): for the older group of patients (grey hexagons and grey line), one normal stage slows down, while in the younger patients (white diamonds and white line) one slows down and another speeds up. Details of the fits can be found in Appendix D5.

In summary, the losses for younger patients are consistent with a stage slowing down, a stage speeding up and a gain change, while those for the older patients show no evidence of a speeding up. The slowing down of the response may be associated with greater spatial integration as ganglion cells and spatial acuity are lost or with the inclusion of additional lateral stages in the processing stream. The differences between the younger and older patients suggests that the compensatory speeding up of the slowed response in younger patients declines with age. Other measures showed relatively little age-dependency. For 
example, the best corrected visual acuity log MAR values \pm 1 S.D. in the eye used for the flicker sensitivity measurements were $0.60 \pm 0.15$ and $0.76 \pm 0.28$ for the younger and older groups, respectively, which are not significantly different. Measures that showed a significant deterioration with age were chromatic thresholds and S-cone flicker acuity (Majander et al., 2017).

\subsection{Cone dystrophy with supernormal rod ERG (KCNV2)}

The next example is from five patients in a group with progressive cone dystrophy caused by pathogenic variants in $K C N V 2$, a gene that encodes a voltage-gated potassiumchannel modifying subunit in the rod and cone photoreceptors (Salah et al., 2008; Thiagalingam et al., 2007; Wissinger et al., 2008; Wu et al., 2006) (see $\mathbf{F}$ in Figure 1). Patients show supernormal rod ERG responses to bright flashes, early reduced central spatial acuity, colour vision disturbances, sometimes night blindness, and often photophobia (e.g., Alexander and Fishman, 1984; Foerster et al., 1990; Gouras et al., 1983; Hood et al., 1996; Kato et al., 1993; Michaelides et al., 2005a; Robson et al., 2010; Rosenberg and Simonsen, 1993; Sandberg et al., 1990; Yagasaki et al., 1986b; Zobor et al., 2012).

[Insert Figure 11 about here]

Figure 11A shows flicker sensitivity data \pm 1 S.E.M. for the five patients: SR1 (blue triangles, 54), SR2 (pink inverted triangles, 35), SR3 (green diamonds, 29), SR4 (dark blue circles, 48) and SR5 (brown hexagons, 44). The sensitivity functions are bandpass in shape with the frequency of peak sensitivity close to normal (near $9 \mathrm{~Hz}$ ), but with substantial losses in sensitivity (except for SR5 and, at low frequencies, SR2). The high-frequency data for all patients follow straight lines. The frequency constants are 36.10 \pm 4.86 (SR1, blue line), 21.36 \pm 3.17 (SR2, pink line), 46.92 \pm 5.96 (SR3, green line), 28.97 \pm 2.96 (SR4, dark blue line) and 17.30 \pm 1.50 (SR5, brown line) $\mathrm{Hz}$. Accordingly, the patients can be split into two groups: SR2 and SR5 have smaller frequency constants that are close to normal $(19.19 \pm 0.42 \mathrm{~Hz})$, while SR1, SR3 and SR4 show much greater frequency constants indicating a faster response.

Figure 11B shows the sensitivity losses for the patients (coloured symbols) relative to the normal sensitivities. The mean losses \pm 1 S.E.M are shown by the yellow dotted squares. The patient losses fall into two distinct categories: the losses for SR2 and SR5 at first increase 
and then flatten at higher frequencies following a function that is characteristic of a stage slowing down. The losses for SR1, SR3 and SR4 also increase at first but then decrease above about $10-15 \mathrm{~Hz}$ thus following the now familiar asymmetrical U-shaped function that is characteristic of one stage slowing down and a second speeding up. These two forms can be accounted for, as before, by taking two normal stages with the same corner frequencies, and in the patients have one slow down and the other either speed up (SR1, SR3, SR4) or stay the same (SR5 and SR2). The individual fits are shown by the solid curves in the panel B. See Appendix D6 for more details of the fits.

In summary, the losses for all five patients are consistent with one stage slowing down and a gain change, but with SR1, SR3, and SR4 also showing evidence of another stage speeding up. Unlike the DOA results in Figure 10, the loss of the compensatory speeding up of the response is not clearly age-related, since it is found in both the youngest and oldest patients.

\subsection{Enhanced S-cone syndrome}

The final example is one patient with a pathogenic variant in NR2E3, a gene that encodes for a photoreceptor-specific nuclear receptor (transcription factor) that causes enhanced Scone syndrome (ESCS), a slowly progressive retinal degeneration (see $\mathbf{G}$ in Figure 1). The syndrome is characterised by night blindness, varying degrees of visual acuity loss, visual field abnormalities and an enhanced S-cone sensitivity; the syndrome is attributed to an excess of S-cones caused by defect in the gene NR2E3 that overpopulates the retina with Scones with fewer L-cones and M-cones and a near absence of rods (e.g., Greenstein et al., 1996; Haider et al., 2000; Hood et al., 1995; Jacobson et al., 1990; Kellner et al., 1993; Marmor et al., 1990; Milam et al., 2002; Sharon et al., 2003). In our original paper, we mainly investigated S-cone flicker sensitivities (Ripamonti et al., 2014a), but made L-cone measurements in one patient (Figure 12) that can be compared with the other results reported here. Although there may be some degeneration, we expect the patient's losses to reflect mainly a reduction in the number of L-cones and their pathways.

[Insert Figure 12 about here] 
Figure 12A shows the flicker sensitivities at the High brightness level for ESC1 (blue circles, aged 37) together with the mean normal data (orange and dashed white lines). The patient's data are like the mean normal data in both shape and sensitivity, suggesting no significant differences in L-cone mediated temporal dynamics. The slope of the line fitted to the patient's high-frequency data has a frequency constant of $18.58 \pm 1.02 \mathrm{~Hz}$ (blue line)very similar to the mean normal frequency constant of $19.19 \pm 0.42 \mathrm{~Hz}$. This patient shows little loss of L-cone function.

\section{Discussion}

In this review, we have looked again at flicker sensitivity measurements made in patients with defects in the GNAT2, RGS9, GUCA1A, RPE65, OPA1, KCNV2 or NR2E3 genes. In each case, we have determined the frequency constants associated with the slopes of the straight lines fitted to each patient's high-frequency data and have reanalysed the flicker sensitivities using the sequential model of visual processing developed by Rider, Henning \& Stockman (2019). That model, which accounts for normal flicker-sensitivity measurements made in different laboratories over the past 65 years, was illustrated in Figure 4 . We have successfully applied the model to the flicker sensitivity losses for each patient or patient group by assuming that their sensitivities depend on the same sequence of processing steps as in normal observers, but that one or more of the steps has been affected by the gene defect. In fact, at most three changes in the normal model have to be made to account for the patient data: speeding of one stage (Figure 5, example A), slowing of one stage (example B), and changes in gain control (example $\mathrm{C}$ ). The quality of the model's fits to both normal and abnormal flicker sensitivity data, as indicated by the low standard error of the estimates (SEE) and by the high $R^{2}$ values, which are given in Tables D1 to D6 in the Appendix, is encouraging. We believe that the best current method of investigating temporal visual disorders psychophysically is to measure flicker sensitivities at both low and high temporal frequencies-and then to apply the sequential model to analyse the losses relative to normal sensitivities. We find that the sequential model, in which we assume that up to three of the steps may be affected by the gene defect, can account well for sensitivity losses in 
every disorder so far measured, and its application has allowed us to distinguish between stages that speed up the visual response, or slow it down, or attenuate it.

We recommend that for measurements made at a single adaptation level, a photopic luminance level comparable to our standard level of $3.25 \log _{10}$ trolands be used, since by that level the speeding up of the visual response due to adaptation is almost complete (see Figure 9 of Rider et al., 2019). Thus, the flicker sensitivities measured at this level will be representative of the fastest visual responses and will extend to the highest temporal frequencies. For direct comparisons with our normative data, a long-wavelength target and short-wavelength background should be used (although the blue background is mainly relevant at lower luminance levels to exclude rod contamination). The number of frequencies measured is also important but will depend on each patient's temporal acuity limit. In normal observers, we measured flicker sensitivities from 0.5 to $45 \mathrm{~Hz}$ : at 0.5 and 1 $\mathrm{Hz}$ and then in 2-Hz steps from 2 to $10 \mathrm{~Hz}$ and in $5-\mathrm{Hz}$ steps from 10 to $45 \mathrm{~Hz}$. Given the inevitable time constraints in making such measurements in patients, the number of frequencies will need to be reduced. For a determination of the frequency constant, three sensitivity measurements at higher frequencies should suffice with the step-size and frequencies depending on the frequency range over which the patient is able to see flicker. For more complete modelling, low and high frequencies should be included with a step-size of about $5 \mathrm{~Hz}$ or less, including frequencies as low as 0.5 or $1 \mathrm{~Hz}$ and as high as can be measured. If the effects of light adaptation are to be investigated, and particularly in cases in which some speeding of the visual response occurs at lower than normal light levels, such as Bradyopsia (RGS9) and dominant progressive cone dystrophy (GUCA1A), lower brightness levels should also be used (taking care to avoid rod contamination).

As we have described above, analyses using the model have contributed important suggestions not only about the clinical disorders and the effects of each molecular loss, but also about normal and abnormal visual processing in general.

Before discussing these analyses further, we consider characterising the disorders in terms of the frequency constant. As we argue in the next section, although a straightforward, efficient, and potentially useful measure of visual performance, the frequency constant is limited in scope because its gauges performance only at higher temporal frequencies. 


\subsection{Frequency constants}

A consistent characteristic of the data for both normal observers and patients is that the fall-off in high-frequency flicker sensitivity with frequency is exponential-as revealed by the consistently good straight-line fits to the high-frequency data plotted as log sensitivity against frequency. The slope of the straight line on these co-ordinates can be measured relatively efficiently with as few as two data points and thus the slope is potentially useful in characterising and monitoring patient performance. We have chosen to specify the slope in terms of the frequency constant; that is, as the change in frequency that reduces sensitivity by a factor of 10 . The higher the frequency constant, the faster the processing and the shallower the slope at the high-frequency end of the flicker sensitivity functions.

\section{[Insert Figure 13 about here]}

In Figure 13, we have plotted the frequency constants at the standard High intensity level for all patients and normal observers. The frequency constants for the normal observers are shown by the yellow circles. Estimated individually, the mean frequency constant for normal observers is $19.51 \mathrm{~Hz}$ with a standard error of $0.41 \mathrm{~Hz}$ (yellow and dashed white lines). The frequency constants for the normal observers and GNAT2 patients (cyan circles) form reasonably distinct clusters. By contrast, those for RPE65 patients (purple hexagons), GUCA1A (green and dotted green triangles), OPA1 (grey and white diamonds), KCNV2 (orange dotted and red inverted triangles), all of whom suffer from progressive disorders, show a greater spread as progressively deteriorating performance reduces the frequency constant.

Frequency constants that fall above the normal cluster are those for patients in whom our modelling suggests that one stage has sped up as a direct or indirect consequence of the gene defect. These include the single RGS9 patient (black square), the younger OPA1 patients (grey diamonds) a subset of KCNV2 patients SR1, SR3 and SR4 (orange dotted inverted triangles), and the youngest GUCA1A patient GP1 (dotted green triangle). Constants that fall below the normal cluster are patients in whom our modelling suggests that one stage has substantially slowed down. These include the GNAT2 patients (blue circles), the RPE65 patients (purple hexagons), and the older GUCA1A patients (green triangles). 
A clear limitation of characterising performance solely in terms of the frequency constant is that it inevitably reflects performance at higher temporal frequencies. Thus, for the RGS9 patient, the younger OPA1 patients and the subset of KCNV2 patients SR1, SR3 and SR4-in all of whom the sequential model shows that the direct effect of the gene defect is to slow down their visual responses-the frequency constants suggest that their responses have sped up, despite temporal sensitivity at higher frequencies being lower than normal. This is because frequency constants probe sensitivities at higher temporal frequencies where, in these patients, the indirect compensatory speeding up of their responses seems to have more effect than the direct effects of the defects.

Despite these limitations, the frequency constant is likely to be useful, for example, in monitoring the loss progression or treatment efficacy. It is remarkable that the frequency constant captures the high-frequency fall in sensitivity across all the clinical disorders we have so far measured. Rider, Henning \& Stockman (2019) showed that this property is an expected characteristic of sequences of low-pass stages with similar corner frequencies-provided that the range of high frequencies is restricted to between about 0.75 to 2 times the corner frequencies of the stages in the processing sequence. If the frequency range exceeds twice the corner frequency, however, the fall in sensitivity will deviate significantly from the approximating exponential form and follow a power-law function with an exponent equal to the number of LP-stages (Rider et al., 2019). According to our model, this deviation should occur, but only at flicker frequencies higher than we can see. The obedience of high-frequency

flicker sensitivity data to the exponential form suggests that both normal and abnormal visual systems are somehow constrained to operate at frequencies less than twice the corner frequencies of their LP-stages.

To understand the disease processes more fully, however, sensitivity losses should be assessed and modelled at both low and high temporal frequencies. Assessment of the frequency constant alone is insufficient.

\subsection{Sequential modelling and abnormal steps}

The results of the modelling are summarised in Table 2 in terms of whether a gene defect causes: $(A)$ a stage to slow down its response; $(B)$ a stage to speed up its response; or (C) a stage 
or stages to attenuate the response. The symbol $\checkmark$ indicates that the modelling supports a change in speed and that it is consistent with a direct effect of the gene defect; whereas $x$ signifies no evidence for a change in speed. The symbols $\checkmark^{*}$ together indicate that the modelling supports a change in speed, but that it is consistent with an indirect effect of the gene defect. The symbol $\square$ indicates that the modelling supports a frequency-independent gain control that could be direct (loss of neural elements, for example) or indirect (compensatory gain adjustments), or both.

\section{TABLE 2}

\begin{tabular}{|c|c|c|c|c|}
\hline \multicolumn{2}{|c|}{ Gene defect } & $\begin{array}{c}\text { (A) } \\
\text { Stage slows down }\end{array}$ & $\begin{array}{c}\text { (B) } \\
\text { Stage speeds up }\end{array}$ & $\begin{array}{c}\text { (C) } \\
\text { Gain control }\end{array}$ \\
\hline \multicolumn{2}{|l|}{ GNAT2 } & $\sqrt{ }$ & $x$ & $\sqrt{ }$ \\
\hline \multicolumn{2}{|l|}{ RGS9 } & $\sqrt{ }$ & $\sqrt{ }$ & $\square$ \\
\hline \multirow{2}{*}{ GUCA1A } & GP1 & $x$ & $\sqrt{ }$ & $\sqrt{ }$ \\
\hline & GP2-4 & $\checkmark$ & $\sqrt{ }$ & $\square$ \\
\hline \multicolumn{2}{|l|}{ RPE65 } & $\sqrt{ }$ & $x$ & $\square$ \\
\hline \multirow{2}{*}{ OPA1 } & $13-39$ & $\checkmark$ & $\checkmark$ & $\sqrt{V}$ \\
\hline & $47-54$ & $\sqrt{ }$ & $x$ & $\sqrt{ }$ \\
\hline \multirow{2}{*}{ KCNV2 } & SR1,3,5 & $\sqrt{ }$ & $V^{*}$ & 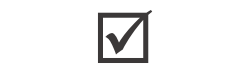 \\
\hline & SR2,4 & $\sqrt{ }$ & $x$ & $\nabla$ \\
\hline \multicolumn{2}{|l|}{$N R 2 E 3$} & $x$ & $x$ & $\square$ \\
\hline
\end{tabular}

Table 2. Summary of stage changes in the eight gene defects listed in Column 1 according to whether a stage slows down the response (column A), speeds it up (column B), or attenuates it (column C). $\checkmark$ alone signifies evidence for a change in speed that is consistent with the direct effect of the gene defect; $x$ signifies no evidence for a change; $\checkmark^{*}$ signifies evidence for a change in speed that is consistent with an indirect effect of the defect; and $\square$ signifies evidence for gain control, that could be direct or indirect.

In most cases, the sensitivity losses are caused by a slowing down of one or more steps of retinal processing $(A)$, which has two effects. The obvious one is that it reduces downstream 
sensitivity to high frequencies. The less obvious one is that this slowing also increases the steady and low-frequency signals passed to downstream processes. This abnormal increase in the steady signal could drive downstream processes into a region less favorable for transmitting information (i.e., saturate them), unless they adapt to the increases (as they would adapt to real increases in light level). Accordingly, the downstream processes adapt by speeding up their responses to reduce the low-frequency signals (B) or by attenuating all frequencies by turning down the overall gain (C). We find examples of both types of adjustment in our data. Gain control is found in all the patient groups and is associated with compensatory speeding up in nearly half of them $(\square)$.

An affected stage that slows down could cause an upstream stage to speed up. All that is required is that a signal downstream from the affected stage feeds back and modifies a stage before the affected stage. A possible example of this can be found in the phototransduction cascade: increases in light level and thus in the rate of photoisomerization lead to a decline in free calcium ions $\left(\mathrm{Ca}^{2+}\right)$ in the cone outer segment that triggers several processes of light adaptation and recovery (Matthews et al., 1988; Nakatani and Yau, 1988). One of these processes is a feedback loop that speeds up the inactivation of light-activated photopigment and thus speeds up the visual response (for details, see, for example, Burns, 2010; Pugh and Lamb, 2000; Zang and Neuhauss, 2018). Thus, an affected stage after the photopigment that causes a decline in free calcium ions can potentially affect the rate of photopigment inactivation. One case in which this feedback loop might be important is that of Bradyopsia (RGS9) in which the direct effect of the genetic variant slows the deactivation of the activated $\alpha$-transducin-PDE6 complex (see above). This in turn will slow down and prolong the visual response, leading to a greater than normal decline in free calcium ions, which through feedback might speed up the preceding photoreceptor response (see, for example, Burns, 2010; Pugh and Lamb, 2000).

Consequently, Bradyopsia is an example where the slowing down of a stage (the deactivation of $\alpha$-transducin-PDE6) may speed up a preceding stage (the deactivation of the active photopigment opsin). The speeding up we find for the Bradyopsia patients may reflect the effect of the feedback loop speeding up the photoreceptor response as well as adaptive rebalancing by later stages. This may explain why the speeding up in this case is excessive and overcompensates for the slow down. 
In the one clear case of a gene defect causing the visual response to speed up (GUCA1A, for patient GP1), which decreases the steady and low-frequency signals passed to downstream processes, we find no evidence of a compensatory slowing down of the visual response. This may simply be because at lower light levels the downstream processes may already be at or near their slowest speeds, so that they cannot slow down any further. In this case, even when the light level rises, the abnormal step may hold the downstream steps at their slowest speeds over a range of light levels. Alternatively, the compensatory adaptational processes may be predominantly aimed at protecting the visual system from saturating, and so only need to speed up.

In developing and fitting the sequential model, we simplified the model by assuming that the speeds of some processing stages varied together with light level while others were fixed (Rider et al., 2019). By directly comparing patient and normal sensitivities-as we have done here, we can begin to tease apart the speeds of individual stages in the processing stream. The function we fit to the differences (given as Equations (A6) or (A7) in the Appendix) include the corner frequencies of both normal and patients, allowing them to be independently estimated from these data.

A frequency-independent gain control $(C)$ is found in all cases $(\square)$. Its origin could be compensatory adjustments that reduce abnormally high signal amplitude, or it could be the result of the loss of neural elements.

\subsection{Underlying physiology and anatomy}

Finally, we consider the sequential model (Figure 4) and speculate how each of the steps might link to the underlying physiology and anatomy, and to the locations of the various gene defects in our patient groups. Fitting this type of linear, sequential model has two major limitations: one theoretical and one empirical. First, the order of the stages in the sequence is not constrained, since the order of the stages could be changed without affecting the predictions of the model. Second, the speeds of individual LP-stages are poorly constrained by flicker-sensitivity data, since increasing the corner frequency of one LP-stage can be largely offset by reducing the corner frequency of another. However, knowledge of which genes are affected in our patient groups allows us to start tentatively to put the 
stages in order; further, comparison of patient and normal data allows us to extract the speeds of some of the individual stages.

We have arranged the model stages in Figure 14 and juxtaposed a simplified diagram of the retina in a pattern that we think is consistent with the underlying physiology. Figure 14 can be thought of as an implementation of the model of Figure 4 but showing four possible "routes" through the model's cascade of stages that are more representative of known retinal physiology. In particular, it incorporates the lateral interactions between nearby cones mediated by horizontal cells and shown in the diagram on the right (for reviews, see Grünert and Martin, 2020; Thoreson and Mangel, 2012). On the left, each possible route begins with the gain control marked $b$ and the numbered pink arrows correspond to the numbered probes shown in Figure 4. Figure 14 is formally equivalent to the model of Figure 4 but more realistic in terms of the underlying physiology.

Tentatively, we suppose that the first three stages [1-3] might be in the cones or their synapses with bipolar cells. The feedforward stages, $[A]$ and $[B]$, with their associated level controls, $k$, and sign inversions are all assumed to lie in lateral connections perhaps mediated by horizontal cells, while step [4] lies in late retina and steps [5] and [6] perhaps after the retina in the cortex. The gain control $b$ is placed early in all pathways where photopigment bleaching can attenuate the input signal, while a secondary gain $g$ is placed later in the pathway, at the point we assume to be the transition from retina to cortex (where the signals change from analogue to pulse trains). It should be noted, however, that having only two sites of gain control is likely to be a simplification and other gain-control processes may be distributed throughout the network produced by disparate mechanisms.

[Insert Figure 14 about here]

Of course, from psychophysical data alone, it is not possible to isolate, locate or characterize individual components of the model and then to associate them with physiological process in the human visual system. The tentative links we propose are based on physiology reviewed by Rider, Henning \& Stockman (2019), and rely especially on the work of Dunn, Lankheet \& Rieke (2007) and Baudin et al. (2019) whose cone-photoreceptor recordings are consistent with impulse responses of a series of three low-pass filtering stages. 


\section{Future directions and conclusions}

We have measured and analyzed flicker sensitivity data in normal observers and in patients with a range of genetic mutations affecting the retina. Our two main approaches have been (i) to quantify the sensitivity losses at higher frequencies using a single measure-the frequency constant and (ii) to account for the sensitivity losses at all frequencies using a sequential model of visual processing.

At higher frequencies, we have consistently found that flicker sensitivity across all observers falls exponentially with frequency, which means that the rate of loss can be characterized by a single number, which we call the "frequency constant". The frequency constant is therefore potentially a very useful measure of visual performance for assessing the extent of loss, disease progression and treatment efficacy in clinical patients, and one that can be efficiently assessed by measuring flicker sensitivities at as few as two frequencies. Its implementation in the clinic will require the development of a relatively simple piece of testing equipment (see Appendix).

We accounted for the losses in patients at all frequencies by applying a simple sequential model in which a small number of parameters vary between patients and normal observers. These parameters can be related to processing steps that in the patient speed up, slow down or attenuate the normal visual response. Some of these changes are the direct result of the molecular loss, but others are secondary effects, such as compensatory adjustments made to offset the effects of the loss, or feedback effects that inappropriately activate the normal processes of light adaptation, or more progressive changes as a genetic variant leads to cell loss.

The application of the sequential model, combined with the known genotypes of our patients, enables the integration of human psychophysics and molecular genetics. The two approaches are complementary. Genetics provides information about the role, function and location of individual proteins in the visual pathway. Psychophysics tells us how genetic variants that affect those proteins change the visual performance of the whole system. So far, we have investigated genetic variants in GNAT2, GUCA1A, and RGS9 that affect the transduction cascade, in KCNV2 that affect photoreceptors after the cascade, and in OPA1 
that affect ganglion cells. We plan to extend measurements to include other genetic variants in the future.

Visual psychophysical models alone provide only limited information about individual processes in the visual pathway. In particular, our multiple-stage sequential model, shown in Figure 4 , is poorly constrained by flicker sensitivity data, so that analysing a set of data in isolation provides a reliable estimate only of the overall speed of all the stages. In contrast, we show that a comparison of two sets of data provides estimates of the speeds of the individual stages that differ between the patient and normal observer as well as the combined speed of the remaining stages that are common to both. By repeating this process with different patient groups in which different stages vary from normal, we have begun to characterise individual stages in the normal and each patient group and build a detailed sequential model. This work will continue in the future as we and hopefully others expand the patient database.

The genetic mutations we study are very rare. Future work should focus on testing as broad a range of patients as possible not only adding patients with the same genetic mutations as those already studied but also adding patients with different mutations. Patients with unknown genetic defects should also be investigated, since the methods and model can identify the nature of their visual losses, and thus suggest the underlying causes and possibly potential mitigations.

These tests necessitate the development of a relatively simple piece of testing equipment that could be based on light-emitting-diodes (see Figure A1 in the Appendix for a simplified experimental set-up) or on a high-refresh-rate, high-brightness, linearized display monitor programmed using an appropriate software package. The experiments require carefully controlled and calibrated stimuli but are not overly laborious or difficult to administer. The general technique is also highly flexible. Here we have focussed on relatively large red targets on larger blue backgrounds that should favour L-cone activation. By varying the wavelengths of the target and background one could, for example, favour and study rod or S-cone mediated vision or examine the properties of the chromatic versus luminance pathways. The spatial properties of the stimuli might also be varied. We show in Appendix B that the flicker sensitivity losses resulting from a slowing down of a visual process is indistinguishable from a reduction in lateral inhibition, caused perhaps by loss of horizontal 
or ganglion cells (compare panels (A) and (D) of Figure 5). By varying the size, or other spatial properties of the flickering target, the effects of losses in lateral inhibition can be teased apart from losses in processing speed.

The integration of human psychophysics and molecular genetics has significant potential for unravelling the details of human visual processing and increasing our understanding of both normal and abnormal vision. It promises to provide more than the sum of its parts. Much more work needs to be done, but these techniques and models provide a clear means of integrating human visual psychophysics with molecular genetics.

\section{Acknowledgements}

The work described has been supported by grants from Fight for Sight, BBSRC, EPSRC, Moorfields Eye Hospital Special Trustees, and the National Institute for Health Research Biomedical Research Centre at Moorfields Eye Hospital NHS Foundation Trust and UCL Institute of Ophthalmology. Or current funding is from the BBSRC (BB/M00211X/1 and BB/R019487/1). We are especially grateful to the patients who willingly gave up so much time to participate in these studies, and without whom this work would not have been possible. We acknowledge the many colleagues and collaborators who contributed to this work and are co-authors in other papers, but especially Rola Ba-Abbad, Jocelyn Cammack, Caterina João, Vy Luong, Anna Majander, Michel Michaelides, Tony Moore, Caterina Ripamonti, Lindsay Sharpe, Hannah Smithson and Andrew Webster. We thank Rola Ba-Abbad, Rhea Eskew, and Omar Mahroo for helpful comments and suggestions.

\section{References}

Alexander, C., Votruba, M., Pesch, U.E., Thiselton, D.L., Mayer, S., Moore, A., Rodriguez, M., Kellner, U., Leo-Kottler, B., Auburger, G., Bhattacharya, S.S., Wissinger, B., 2000. OPA1, encoding a dynamin-related GTPase, is mutated in autosomal dominant optic atrophy linked to chromosome 3q28. Nat Genet 26, 211-215.

Alexander, K.R., Fishman, G.A., 1984. Supernormal scotopic ERG in cone dystrophy. Brit J Ophthalmol 68, 69-78. 
Arshavsky, V.Y., Lamb, T.D., Pugh, E.N., Jr., 2002. G proteins and phototransduction. Annual Review of Physiology 64, 153-187.

Bainbridge, J.W.B., Mehat, M.S., Sundaram, V., Robbie, S.J., Barker, S.E., Ripamonti, C., Georgiadis, A., Mowat, F.M., Beattie, S.G., Gardner, P.J., Feathers, K.L., Luong, V.A., Yzer, S., Balaggan, K., Viswanathan, A., de Ravel, T.J.L., Casteels, I., Holder, G.E., Tyler, N., Fitzke, F.W., Weleber, R.G., Nardini, M., Moore, A.T., Thompson, D.A., Petersen-Jones, S.M., Michaelides, M., van den Born, L.I., Stockman, A., Smith, A.J., Rubin, G.S., Ali, R.R., 2015. Long-Term Effect of Gene Therapy on Leber's Congenital Amaurosis. New Engl J Med 372, 1887-1897.

Bainbridge, J.W.B., Smith, A.J., Barker, S.S., Robbie, S., Henderson, R., Balaggan, K., Viswanathan, A., Holder, G.E., Stockman, A., Tyler, N., Petersen-Jones, S., Bhattacharya, S.S., Thrasher, A.J., Fitzke, F.W., Carter, B.J., Rubin, G.S., Moore, A.T., Ali, R.R., 2008. Effect of gene therapy on visual function in Leber's congenital amaurosis. New Engl J Med 358, 1-9.

Barboni, P., Savini, G., Cascavilla, M.L., Caporali, L., Milesi, J., Borrelli, E., La Morgia, C., Valentino, M.L., Triolo, G., Lembo, A., Carta, A., De Negri, A., Sadun, F., Rizzo, G., Parisi, V., Pierro, L., Bianchi Marzoli, S., Zeviani, M., Sadun, A.A., Bandello, F., Carelli, V., 2014. Early macular retinal ganglion cell loss in dominant optic atrophy: genotype-phenotype correlation. Am J Ophthalmol 158, 628-636.

Barlow, H.B., Levick, W.R., 1976. Threshold setting by the surround of cat retinal ganglion cells. Journal of Physiology 259, 737-757.

Barten, P.G.J., 1999. Contrast sensitivity of the human eye and its effects on image quality. Technische Universiteit Eindhoven, Eindhoven.

Baudin, J., Angueyra, J.M., Sinha, R., Rieke, F., 2019. S-cone photoreceptors in the primate retina are functionally distinct from $\mathrm{L}$ and $\mathrm{M}$ cones. eLife 8, e39166.

Berger, W., Kloeckener-Gruissem, B., Neidhardt, J., 2010. The molecular basis of human retinal and vitreoretinal diseases. Prog Retin Eye Res 29, 335-375.

Bills, M.A., 1920. The lag of visual sensation in its relation to wave lengths and intensity of light. Psychological review company, Princeton, N.J., Lancaster, Pa.,. 
Burns, M.E., 2010. Deactivation Mechanisms of Rod Phototransduction The Cogan Lecture. Invest Ophth Vis Sci 51, 1283-1288.

Burns, M.E., Baylor, D.A., 2001. Activation, deactivation and adaptation in vertebrate photoreceptor cells. Annual review of neuroscience 24, 779-805.

Burte, F., Carelli, V., Chinnery, P.F., Yu-Wai-Man, P., 2015. Disturbed mitochondrial dynamics and neurodegenerative disorders. Nature Reviews Neurology 11, 11-24.

Cheng, J.Y.C., Luu, C.D., Yong, V.H.K., Mathur, R., Aung, T., Vithana, E.N., 2007. Bradyopsia in an Asian man. Arch Ophthalmol-Chic 125, 1138-1140.

Cohn, A.C., Toomes, C., Hewitt, A.W., Kearns, L.S., Inglehearn, C.F., Craig, J.E., Mackey, D.A., 2008. The natural history of OPA1-related autosomal dominant optic atrophy. Brit J Ophthalmol 92, 1333-1336.

de Lange, H., 1952. Experiments on flicker and some calculations on an electrical analogue of the foveal systems. Physica 18, 935-950.

de Lange, H., 1958. Research into the dynamic nature of the human fovea-cortex systems with intermittent and modulated light. I. Attenuation characteristics with white and colored light. J Opt Soc Am 48, 777-784.

de Lange, H., 1961. Eye's response at flicker fusion to square-wave modulation of a test field surrounded by a large steady field of equal mean luminance. J Opt Soc Am 51, 415-421.

Delettre, C., Lenaers, G., Griffoin, J.M., Gigarel, N., Lorenzo, C., Belenguer, P., Pelloquin, L., Grosgeorge, J., Turc-Carel, C., Perret, E., Astarie-Dequeker, C., Lasquellec, L., Arnaud, B., Ducommun, B., Kaplan, J., Hamel, C.P., 2000. Nuclear gene OPA1, encoding a mitochondrial dynamin-related protein, is mutated in dominant optic atrophy. Nat Genet 26, 207-210.

Downes, S.M., Holder, G.E., Fitzke, F.W., Payne, A.M., Warren, M.J., Bhattacharya, S.S., Bird, A.C., 2001. Autosomal dominant cone and cone-rod dystrophy with mutations in the guanylate cyclase activator $1 \mathrm{~A}$ gene-encoding guanylate cyclase activating protein-1. Arch Ophthalmol-Chic 119, 96-105.

Dunn, F.A., Lankheet, M.J., Rieke, F., 2007. Light adaptation in cone vision involves switching between receptor and post-receptor sites. Nature 449, 603-607. 
Fain, G.L., Matthews, H.R., Cornwall, M.C., Koutalos, Y., 2001. Adaptation in vertebrate photoreceptors. Physiological Reviews 80, 117-151.

Ferry, E.S., 1892. Persistence of vision. American Journal of Science 44, 192-207.

Foerster, M.H., Kellner, U., Wessing, A., 1990. Cone dystrophy and supernormal darkadapted b-waves in the electroretinogram. Graefes Archives of Clinical and Experimental Ophthalmology 228, 116-119.

Geisler, W.S., 1978. The effects of photopigment depletion on brightness and threshold. Vision Res 18, 269-278.

Gouras, P., Eggers, H.M., MacKay, C.J., 1983. Cone dystrophy, nyctalopia, and supernormal rod responses. A new retinal degeneration. Arch Ophthalmol-Chic 101, 718-724.

Greenstein, V.C., Zaidi, Q., Hood, D.C., Spehar, B., Cideciyan, A.V., Jacobson, S.G., 1996. The enhanced S cone syndrome: an analysis of receptoral and post-receptoral changes. Vision Res 36, 3711-3722.

Grünert, U., Martin, P.R., 2020. Cell types and cell circuits in human and non-human primate retina. Prog Retin Eye Res 78, 100844.

Gu, S.M., Thompson, D.A., Srikumari, C.R., Lorenz, B., Finckh, U., Nicoletti, A., Murthy, K.R., Rathmann, M., Kumaramanickavel, G., Denton, M.J., Gal, A., 1997. Mutations in RPE65 cause autosomal recessive childhood-onset severe retinal dystrophy. Nat Genet 17, 194197.

Haider, N.B., Jacobson, S.G., Cideciyan, A.V., Swiderski, R., Streb, L.M., Searby, C., Beck, G., Hockey, R., Hanna, D.B., Gorman, S., Duhl, D., Carmi, R., Bennett, J., Weleber, R.G., Fishman, G.A., Wright, A.F., Stone, E.M., Sheffield, V.C., 2000. Mutation of a nuclear receptor gene, NR2E3, causes enhanced S cone syndrome, a disorder of retinal cell fate. Nat Genet 24, 127131.

Hanein, S., Perrault, I., Gerber, S., Tanguy, G., Barbet, F., Ducroq, D., Calvas, P., Dollfus, H., Hamel, C.P., Lopponen, T., Munier, F., Santos, L., Shalev, S., Zafeirou, D., Dufier, J.L., Munnich, A., Rozet, J.M., Kaplan, J., 2004. Leber congenital amaurosis: comprehensive survey of the genetic heterogeneity, refinement of the clinical definition, and genotypephenotype correlations as a strategy for molecular diagnosis. Hum Mutat 23, 306-317. 
Hartong, D.T., Pott, J.W.R., Kooijman, A.C., 2007. Six patients with bradyopsia (slow vision) Clinical features and course of the disease. Ophthalmology 114, 2323-2331.

Hood, D.C., Cideciyan, A.V., Halevy, D.A., Jacobson, S.G., 1996. Sites of disease action in a retinal dystrophy with supernormal and delayed rod electroretinogram $b$-waves. Vision Res $36,889-901$.

Hood, D.C., Cideciyan, A.V., Román, A.J., Jacobson, S.G., 1995. Enhanced S cone syndrome: evidence for an abnormally large number of S cones. Vision Res 35, 1473-1481.

Ives, H.E., 1922. A theory of intermittent vision. J Opt Soc Am 6, 343-361.

Jacobson, S.G., Marmor, M.F., Kemp, C.M., Knighton, R.W., 1990. SWS (blue) cone hypersensitivity in a newly identified retinal degeneration. Investigative Ophthalmololy \& Visual Science 31, 827-838.

Jacobson, S.G., Voigt, W.J., Parel, J.M., Apathy, P.P., Nghiem-Phu, L., Myers, S.W., Patella, V.M., 1986. Automated light- and dark-adapted perimetry for evaluating retinitis pigmentosa. Ophthalmology 93, 1604-1611.

Johnston, P.B., Gaster, R.N., Smith, V.C., Tripathi, R.C., 1979. A clinicopathologic study of autosomal dominant optic atrophy. Am J Ophthalmol 88, 868-675.

Kato, M., Kobayashi, R., Watanabe, I., 1993. Cone dysfunction and supernormal scotopic electroretinogram with a high-intensity stimulus. A report of three cases. Doc Ophthalmol $84,71-81$.

Kellner, U., Zrenner, E., Sadowski, B., Foerster, M.H., 1993. Enhanced S cone sensitivity syndrome: long-term follow-up, electrophysiological and psychophysical findings. Clin Vision Sci 8, 425-434.

Kelly, D.H., 1961. Visual responses to time-dependent stimuli I. Amplitude sensitivity measurements. J Opt Soc Am 51, 422-429.

Kjer, P., 1959. Infantile optic atrophy with dominant mode of inheritance: a clinical and genetic study of 19 Danish families. Acta Ophthalmologica Supplement 164, 1-147.

Kjer, P., Jensen, O.A., Klinken, L., 1983. Histopathology of eye, optic nerve and brain in a case of dominant optic atrophy. Acta Ophthalmol 61, 300-312. 
Kulikowski, J.J., 1971. Some stimulus parameters affecting spatial and temporal resolution of human vision. Vision Res 11, 83-93.

Lamb, T.D., Pugh, E.N., Jr., 2004. Dark adaptation and the retinoid cycle of vision. Prog Retin Eye Res 23, 307-380.

Leibrock, C.S., Reuter, T., Lamb, T.D., 1998. Molecular basis of dark adaptation in rod photoreceptors. Eye 12, 511-520.

Levenberg, K., 1944. A method for the solution of certain non-linear problems in least squares. Quarterly of Applied Mathematics 2, 164-168.

MacLeod, D.I.A., 1978. Visual sensitivity. Annual Review of Psychology 29, 613-645.

Majander, A., João, C., Rider, A.T., Henning, G.B., Votruba, M., Moore, A.T., Yu-Wai-Man, P., Stockman, A., 2017. The pattern of retinal ganglion cell loss in OPA1-related autosomal dominant optic atrophy inferred from temporal, spatial, and chromatic sensitivity losses. Invest Ophth Vis Sci 58, 502-516.

Marlhens, F., Bareil, C., Griffoin, J.M., Zrenner, E., Amalric, P., Eliaou, C., Liu, S.Y., Harris, E., Redmond, T.M., Arnaud, B., Claustres, M., Hamel, C.P., 1997. Mutations in RPE65 cause Leber's congenital amaurosis. Nat Genet 17, 139-141.

Marmor, M.F., Jacobson, S.G., Forester, M.H., Kellner, U., Weleber, R.G., 1990. Diagnostic findings of a new syndrome with night blindness, maculopathy, and enhanced S cone sensitivity. Am J Ophthalmol 110, 124-134.

Marquardt, D.W., 1963. An algorithm for least-squares estimation of nonlinear parameters. Journal of the Society for Industrial and Applied Mathematics 11, 431-441.

Matin, L., 1968. Critical duration, the differential luminance threshold, critical flicker frequency, and visual adaptation: a theoretical treatment. J Opt Soc Am 58, 404-415. Matthews, H.R., Murphy, R.L.W., Fain, G.L., Lamb, T.D., 1988. Photoreceptor light adaptation is mediated by cytoplasmic calcium concentration. Nature 334, 67-69. Michaelides, M., Hardcastle, A.J., Hunt, D.M., Moore, A.T., 2006. Progressive cone and conerod dystrophies: phenotypes and underlying molecular genetic basis. Surv Ophthalmol 51, 232-258. 
Michaelides, M., Holder, G.E., Webster, A.R., Hunt, D.M., Bird, A.C., Fitzke, F.W., Mollon, J.D., Moore, A.T., 2005a. A detailed phenotypic study of "cone dystrophy with supernormal rod ERG". Brit J Ophthalmol 89, 332-339.

Michaelides, M., Li, Z., Rana, N.A., Richardson, E.C., Hykin, P.G., Moore, A.T., Holder, G.E., Webster, A.R., 2010. Novel Mutations and Electrophysiologic Findings in RGS9-and R9APAssociated Retinal Dysfunction (Bradyopsia). Ophthalmology 117, 120-U151.

Michaelides, M., Wilkie, S.E., Jenkins, S., Holder, G.E., Hunt, D.M., Moore, A.T., Webster, A.R., 2005b. Mutation in the gene GUCA1A, encoding guanylate cyclase-activating protein 1, causes cone, cone-rod, and macular dystrophy. Ophthalmology 112, 1442-1447.

Milam, A.H., Rose, L., Cideciyan, A.V., Barakat, M.R., Tang, W.-X., Gupta, N., Aleman, T.S., Wright, A.F., Stone, E.M., Sheffield, V.C., Jacobson, S.G., 2002. The nuclear receptor NR2E3 plays a role in human retinal photoreceptor differentiation and degeneration. Proceedings of the National Academy of Sciences 99, 473-478.

Milea, D., Sander, B., Wegener, M., Jensen, H., Kjer, B., Jorgensen, T.M., Lund-Andersen, H., Larsen, M., 2010. Axonal loss occurs early in dominant optic atrophy. Acta Ophthalmol 88, 342-346.

Nakatani, K., Yau, K.W., 1988. Calcium and light adaptation in retinal rods and cones. Nature $334,69-71$.

Nishiguchi, K.M., Sandberg, M.A., Kooijman, A.C., Martemyanov, K.A., Pott, J.W.R., Hagstrom, S.A., Arshavsky, V.Y., Berson, E.L., Dryja, T.P., 2004. Defects in RGS9 or its anchor protein R9AP in patients with slow photoreceptor deactivation. Nature 427, 75-78.

Parinaud, H., 1881. L'héméralopie et les fonctions du pourple visuel. Comptes rendus hebdomadaires des séances et mémories de la Société de biologie 93, 286-287.

Payne, A.M., Downes, S.M., Bessant, D.A., Taylor, R., Holder, G.E., Warren, M.J., Bird, A.C., Bhattacharya, S.S., 1998. A mutation in guanylate cyclase activator 1A (GUCA1A) in an autosomal dominant cone dystrophy pedigree mapping to a new locus on chromosome 6p21.1. Hum Mol Genet 7, 273-277.

Perlman, I., Normann, R.A., 1998. Light adaptation and sensitivity controlling mechanisms in vertebrate photoreceptors. Prog Retin Eye Res 17, 523-563. 
Perrault, I., Rozet, J.M., Gerber, S., Ghazi, I., Leowski, C., Ducroq, D., Souied, E., Dufier, J.L., Munnich, A., Kaplan, J., 1999a. Leber congenital amaurosis. Molecular Genetics and Metabolism 68, 200-208.

Perrault, I., Rozet, J.M., Ghazi, I., Leowski, C., Bonnemaison, M., Gerber, M., Ducroq, D., Cabot, A., Souied, E., Dufier, J.L., Pittler, S., Munnich, A., Kaplan, J., 1999b. Different functional outcome of retGC1 and RPE65 gene mutations in Leber congenital amaurosis. Am J Hum Genet 64, 1225-1228.

Pianta, M.J., Kalloniatis, M., 2000. Characterisation of dark adaptation in human cone pathways: an application of the equivalent background hypothesis. Journal of Physiology $528,591-608$.

Pontikos, N., Arno, G., Jurkute, N., Schiff, E., Ba-Abbad, R., Malka, S., Gimenez, A., Georgiou, M., Wright, G.A., Armengol, M., Knight, H., Katz, M., Moosajee, M., Yu-Wai-Man, P., Moore, A.T., Michaelides, M., Webster, A.R., Mahroo, O.A., 2020. Genetic basis of inherited retinal disease in a molecularly characterized cohort of more than 3000 families from the United Kingdom. Ophthalmology 127, 1384-1394.

Porter, T.C., 1902. Contributions to the study of flicker. Paper II. P R Soc London 70, 313-329. Pugh, E.N., Jr., Lamb, T.D., 2000. Phototransduction in vertebrate rods and cones: molecular mechanisms of amplification, recovery and light adaptation, in: Stavenga, D.G., de Grip, W.J., Pugh, E.N. (Eds.), Handbook of biological physics, Vol. 3, Molecular mechanisms of visual transduction. Elsevier, Amsterdam, pp. 183-255.

Pugh, E.N., Jr., Nikonov, S., Lamb, T.D., 1999. Molecular mechanisms of vertebrate photoreceptor light adaptation. Current Opinion in Neurobiology 9, 410-418.

Pulfrich, C., 1922. Die Stereskopie im Dienste der isochromen und heterochromen Photometrie. Naturwissenschaften 10, 553-564.

Purpura, K., Tranchina, D., Kaplan, E., Shapley, R.M., 1990. Light adaptation in the primate retina: Analysis of changes in gain and dynamics of monkey retinal ganglion cells. Visual Neurosci 4, 75-93. 
Redmond, T.M., Yu, S., Lee, E., Bok, D., Hamasaki, D., Chen, N., Goletz, P., Ma, J.X., Crouch, R.K., Pfeifer, K., 1998. Rpe65 is necessary for production of 11-cis-vitamin A in the retinal visual cycle. Nat Genet 20, 344-351.

Rider, A.T., Henning, G.B., Stockman, A., 2019. Light adaptation controls visual sensitivity by adjusting the speed and gain of the response to light. Plos One 14, e0220358.

Ripamonti, C., Aboshiha, J., Henning, G.B., Sergouniotis, I.P., Michaelides, M., Moore, A.T., Webster, A., Stockman, A., 2014a. Vision in observers with enhanced S-cone syndrome: an excess of S-cones but connected mainly to conventional S-cone pathways. Investigative Ophthalmology and Visual Science 55, 963-976.

Ripamonti, C., Henning, G.B., Ali, R.R., Bainbridge, J.W.B., Robbie, S.J., Sundaram, V., Luong, V.A., van den Born, L.I., Casteels, I., de Ravel, T.J.L., Moore, A.T., Stockman, A., 2014b. Nature of the visual loss in observers with Leber's congenital amaurosis caused by specific mutations in RPE65. Invest Ophth Vis Sci 55, 6817-6828.

Robson, A.G., Webster, A.R., Michaelides, M., Downes, S.M., Cowling, J.A., Hunt, D.M., Moore, A.T., Holder, G.E., 2010. Cone dystrophy with supernormal rod electroretinogram: a comprehensive genotype/phenotype study including fundus autofluorescence and extensive electrophysiology. Retina, the journal of retinal and vitreous diseases 30, 51-62.

Rogers, B.J., Anstis, S.M., 1972. Intensity versus adaptation and the Pulfrich stereophenomenon. Vision Res 12, 909-928.

Roman, A.J., Cideciyan, A.V., Aleman, T.S., Jacobson, S.G., 2007. Full-field stimulus testing (FST) to quantify visual perception in severely blind candidates for treatment trials. Physiological Measurement 28, N51-N56.

Ronnback, C., Milea, D., Larsen, M., 2013. Imaging of the macula indicates early completion of structural deficit in autosomal-dominant optic atrophy. Ophthalmology 120, 2672-2677. Ronnback, C., Nissen, C., Almind, G.J., Gronskov, K., Milea, D., Larsen, M., 2015. Genotypephenotype heterogeneity of ganglion cell and inner plexiform layer deficit in autosomaldominant optic atrophy. Acta Ophthalmol Scan 93, 762-766.

Rosenberg, T., Simonsen, S.E., 1993. Retinal cone dysfunction of supernormal rod ERG type. Five new cases. Acta Ophthalmol 71, 246-255. 
Roufs, J.A.J., 1972a. Dynamic properties of vision-I. Experimental relationships between flicker and flash thresholds. Vision Res 12, 261-278.

Roufs, J.A.J., 1972b. Dynamic properties of vision. II. Theoretical relationship between flicker and flash thresholds. Vision Res 12, 279-292.

Rovamo, J., Raninen, A., Donner, K., 1999. The effects of temporal noise and retinal luminance on foveal flicker sensitivity. Vision Res 39, 533-539.

Salah, S.B., Kamei, S., Sénéćhal, A., Lopez, S., Bazalgette, C., Bazalgette, C., Eliaou, C.M., Zanlonghi, X., Hamel, C.P., 2008. Novel KCNV2 mutations in cone dystrophy with supernormal rod electroretinogram. Am J Ophthalmol 145, 1099-1106.

Sandberg, M.A., Miller, S., Berson, E.L., 1990. Rod electroretinograms in an elevated cyclic guanosine monophosphate-type human retinal degeneration. Comparison with retinitis pigmentosa. Investigative Ophthalmology and Visual Science 31, 2283-2287.

Schade, O.N., 1956. Optical and photoelectric analog of the eye. J Opt Soc Am 46, 721-738. Schultze, M., 1866. Zur Anatomie und Physiologie der Retina. Archiv für mikroskopische Anatomie und Entwicklungsmechanik 2, 175-286.

Shapley, R., 2009. Linear and nonlinear systems analysis of the visual system: why does it seem so linear? A review dedicated to the memory of Henk Spekreijse. Vision Res 49, 907921.

Shapley, R., Enroth-Cugell, C., 1984. Visual Adaptation and Retinal Gain Controls. Progress in Retinal Research 3, 263-346.

Sharon, D., Sandberg, M.A., Caruso, R.C., Berson, E.L., Dryja, T.P., 2003. Shared mutations in NR2E3 in enhanced S-cone syndrome, Goldmann-Favre syndrome, and many cases of clumped pigmentary retinal degeneration. Arch Ophthalmol-Chic 121, 1316-1323.

Simunovic, M.P., Moore, A.T., MacLaren, R.E., 2016. Selective automated perimetry under photopic, mesopic, and scotopic conditions: Detection mechanisms and testing strategies. Translational Visual Science \& Technology 5, 10.

Sperling, G., Sondhi, M.M., 1968. Model for visual luminance discrimination and flicker detection. J Opt Soc Am 58, 1133-1145. 
Stiles, W.S., Crawford, B.H., 1932. Equivalent adaptation levels in localised retinal areas., in: Rankine, A.O., Ferguson, A. (Eds.), Report of a joint discussion on vision held on June 3, 1932, at the Imperial College of Science by the Physical and Optical Societies. Cambridge University Press, Cambridge, pp. 194-211.

Stockman, A., Brainard, D.H., 2015. Fundamentals of color vision I: Color processing in the eye, in: Elliot, A.J., Fairchild, M.D., Franklin, A. (Eds.), Handbook of Color Psychology. Cambridge University Press, Cambridge, pp. 27-69.

Stockman, A., Henning, G.B., Michaelides, M., Moore, A.T., Webster, A., Cammack, J., Ripamonti, C., 2014a. Cone dystrophy with "supernormal” rod ERG: psychophysical testing shows comparable rod and cone temporal sensitivity losses with no gain in rod function. Invest Ophth Vis Sci 55, 832-840.

Stockman, A., Henning, G.B., Moore, A.T., Webster, A.R., Michaelides, M., Ripamonti, C., 2014b. Visual consequences of molecular changes in the guanylate cyclase activating protein. Invest Ophth Vis Sci 55, 1930-1940.

Stockman, A., Langendörfer, M., Smithson, H.E., Sharpe, L.T., 2006. Human cone light adaptation: from behavioral measurements to molecular mechanisms. J Vision 6, 11941213.

Stockman, A., Sharpe, L.T., 2000. Spectral sensitivities of the middle- and long-wavelength sensitive cones derived from measurements in observers of known genotype. Vision Res 40, 1711-1737.

Stockman, A., Sharpe, L.T., Tufail, A., Kell, P.D., Ripamonti, C., Jeffery, G., 2007a. The effect of sildenafil citrate (Viagra ${ }^{\circledR}$ ) on visual sensitivity. J Vision 7(8):4, 4, 1-15.

Stockman, A., Smithson, H.E., Michaelides, M., Moore, A.T., Webster, A.R., Sharpe, L.T., 2007b. Residual cone vision without $\alpha$-transducin. J Vision 7, 8.1-13.

Stockman, A., Smithson, H.E., Webster, A.R., Holder, G.E., Rana, N.A., Ripamonti, C., Sharpe, L.T., 2008. The loss of the PDE6 deactivating enzyme, RGS9, results in precocious light adaptation at low light levels. J Vision 8, 10.11-10. 
Swanson, W.H., Ueno, T., Smith, V.C., Pokorny, J., 1987. Temporal modulation sensitivity and pulse-detection thresholds for chromatic and luminance perturbations. J. Opt. Soc. Am. A 4, 1992-2005.

Thiagalingam, S., McGee, T.L., Sandberg, M.A., Trzupek, K.M., Berson, E.L., Dryja, T.P., 2007. Novel mutations in the KCNV2 gene in patients with cone dystrophy and a supernormal rod electroretinogram. Ophthalmic Genet 28, 135-142.

Thoreson, W.B., Mangel, S.C., 2012. Lateral interactions in the outer retina. Prog Retin Eye Res 31, 407-441.

Tranchina, D., Gordon, J., Shapley, R.M., 1984. Retinal Light Adaptation - Evidence for a Feedback Mechanism. Nature 310, 314-316.

von Wiegand, T.E., Graham, N., Hood, D.C., 1995. Testing a computational model of lightadaptation dynamics. Vision Res 35, 3037-3051.

Votruba, M., Fitzke, F.W., Holder, G.E., Carter, A., Bhattacharya, S.S., Moore, A.T., 1998. Clinical features in affected individuals from 21 pedigrees with dominant optic atrophy. Arch Ophthalmol-Chic 116, 351-358.

Wang, J.-S., Kefalov, V.J., 2011. The cone-specific visual cycle. Prog Retin Eye Res 30, 115128.

Watson, A.B., 1986. Temporal sensitivity, in: Boff, K., Kaufman, L., Thomas, J. (Eds.), Handbook of Perception and Human Performance. Wiley, New York, pp. 6-1-6-43.

Watson, A.B., Ahumada, A.J., Jr, 2015. Extending the flicker visibility metric to a range of mean luminance. SID Symposium Digest of Technical Papers 46, 30-32.

Westheimer, G., 1966. The Maxwellian view. Vision Res 6, 669-682.

Wissinger, B., Dangel, S., Jägle, H., Hansen, L., Baumann, B., Rudolph, G., Wolf, C., Bonin, M., Koeppen, K., Ladewig, T., Kohl, S.h., Zrenner, E., Rosenberg, T., 2008. Cone dystrophy with supernormal rod response is strictly associated with mutations in KCNV2. Investigative Ophthalmology and Visual Science 49, 751-757. 
Wu, B.X., Moiseyev, G., Chen, Y., Rohrer, B., Crouch, R.K., Ma, J.X., 2004. Identification of RDH10, an all-trans retinol dehydrogenase, in retinal Muller cells. Invest Ophth Vis Sci 45, 3857-3862.

Wu, H., Cowing, J.A., Michaelides, M., Wilkie, S.E., Jeffery, G., Jenkins, S.A., Mester, V., Bird, A.C., Robson, A.G., Holder, G.E., Moore, A.T., Hunt, D.M., Webster, A.R., 2006. Mutations in the gene KCNV2 encoding a voltage-gated Potassium channel subunit cause "cone dystrophy with supernormal rod electroretinogram" in humans. Am J Hum Genet 79, 574579.

Yagasaki, K., Miyake, Y., Awaya, S., Ichikawa, H., 1986a. ERG (electroretinogram) in hereditary optic atrophies. Nippon Ganka Gakkai Zasshi 90, 124-130.

Yagasaki, K., Miyake, Y., Litao, R.E., Ichikawa, K., 1986b. Two cases of retinal degeneration with an unusual form of electroretinogram. Documenta Ophthalmogica 63, 73-82.

Yu-Wai-Man, P., Griffiths, P.G., Burke, A., Sellar, P.W., Clarke, M.P., Gnanaraj, L., Ah-Kine, D., Hudson, G., Czermin, B., Taylor, R.W., Horvath, R., Chinnery, P.F., 2010. The prevalence and natural history of dominant optic atrophy due to OPA1 mutations. Ophthalmology 117, 1538-1546,.

Zang, J., Neuhauss, S.C.F., 2018. The binding properties and physiological functions of Recoverin. Frontiers in Molecular Neuroscience 11.

Znoiko, S.L., Crouch, R.K., Moiseyev, G., Ma, J.X., 2002. Identification of the RPE65 protein in mammalian cone photoreceptors. Invest Ophth Vis Sci 43, 1604-1609.

Zobor, D., Kohl, S., Wissinger, B., Zrenner, E., Jägle, H., 2012. Rod and cone function in patients with KCNV2 retinopathy. Plos One 7, e46762. 


\section{Appendix A. Methodological details}

\section{A1. Experimental system}

The optical system that we used for our experiments is known as a "Maxwellian-view" system (see Westheimer, 1966). Figure A1 is a diagram of the essential features of a "twochannel" Maxwellian-view system. The two light sources separately illuminate each channel, and the beamsplitter (which reflects half and transmits half of the light across its diagonal internal surface) combines them. The lenses in each channel first collimate the light and then image the light sources onto the pupil, while the final lens in the common pathwaythe "Maxwellian" lens-together with the optics of the eye image the light-filled target and background apertures (which correspond to what we see) onto the retina. In our experiments, the target aperture forms a disc on the retina that is $4^{\circ}$ in visual diameter and is illuminated by a red 650-nm light that is optically superimposed onto a second disc formed on the retina by the $9^{\circ}$ background aperture that is illuminated by a blue $481-\mathrm{nm}$ light. The observer's view is of a red target disc centred in a blue circular disc (see inset). The main advantage of a Maxwellian-view system is that it can produce high-intensity, uniform images directly on the retina.

Calibration is also straightforward since it only involves measuring the light entering the pupil and knowing the retinal area over which the light falls. We measure intensity in units of quanta per second per degree squared (quanta s ${ }^{-1} \mathrm{deg}^{-2}$ ). So, for example, for a $650-\mathrm{nm}$ target of $9.0 \log _{10}$ quanta $\mathrm{s}^{-1} \mathrm{deg}^{-2}$ and $4^{\circ}$ in visual diameter (i.e., an area on the retina of $12.57 \mathrm{deg}^{2}$ ), the number of quanta entering the pupil is $12,570,000,000$ per second. We used a Xenon-arc lamp as our light source, but LEDs could be used instead to carry out the experiments described here with the intensity of the red LED being temporally modulated under electronic or computer control. One caveat is that the images of the light sources in the pupil must fall within the smallest natural pupil to avoid the intensity being reduced by pupillary constriction at higher light levels.

\section{[Insert Figure A1 about here]}

The combination of a 650-nm target and a 481-nm background favors flicker detection by the long-wavelength-sensitive L-cones, which are more sensitive than the middle- 
wavelength-sensitive $M$-cones to the $650-\mathrm{nm}$ light and less sensitive to the $481-\mathrm{nm}$ background (Stockman and Sharpe, 2000). The combination also makes rod involvement in flicker detection unlikely. Restricting flicker detection to largely L-cones potentially simplifies the interpretation of the results.

In our experiments, the intensities of the lights were controlled by inserting fixed and variable filters (not shown) in the collimated beams that attenuate the light. Additionally, a fast ferro-electric liquid crystal shutter (Displaytech, Longmont, $\mathrm{CO}$; also not shown) in the target channel was used to vary the intensity of the 650-nm light sinusoidally at various temporal frequencies. The sinusoidal variation was produced by pulse-width modulation of the liquid-crystal shutter at a carrier frequency of $400 \mathrm{~Hz}$ (which is much too fast to be resolved), so that the observers saw only the sinusoidal modulation of the light produced by varying the pulse width. The maximum flicker contrast of $92 \%$ was limited by the rise and fall times of the shutter, which were less than $50 \mu \mathrm{s}$. Under computer control, the flicker amplitude could be varied while keeping the time-averaged intensity constant. The intensities of the lights are given as $\log _{10}$ quanta s $\mathrm{s}^{-1} \mathrm{deg}^{-2}$. The blue, 481-nm background was fixed at about $8.20 \log _{10}$ quanta $\mathrm{s}^{-1} \mathrm{deg}^{-2}$ (which is $2.46 \log _{10}$ scotopic trolands or $1.31 \log _{10}$ photopic trolands). The background was present for all the experiments reported here. Wavelengths were selected by the use of interference filters with full-width at halfmaximum bandwidths of $10 \mathrm{~nm}$ (Ealing Corporation, Holliston, MA, or Oriel, Stratford, CT).

\section{A2. Procedures}

All observers light adapted to the background and target for 3 minutes before any measurements. Each experiment was repeated three times usually on separate days. The means of the results for each experimental run were averaged across runs and the standard error determined. For the flicker sensitivity measurements, the mean intensity of the flickering target was fixed at a given radiance: $7.45,8.42,9.41$ or $10.38 \log _{10}$ quanta s $\mathrm{seg}^{-2}$ (or $0.33,1.29,2.28$ and $3.24 \log _{10}$ photopic trolands), corresponding to the Low, Mediumlow, Medium-high and High levels. The frequency of the sinusoidally flickering target was also fixed at a value ranging from 0.5 to $50 \mathrm{~Hz}$ depending on the conditions and the observer. At each mean intensity and frequency, the observer adjusted the amplitude of the 
flickering stimulus to determine the lowest amplitude at which flicker was just visible (see Figure $2 \mathrm{C})$.

For all measurements, settings were made on each of three separate sessions for the normal observers and averaged. For the patients, depending on availability and time constraints, three settings were made and averaged on each of one, two or three separate sessions. The standard errors shown in the data plots for individual observers show the variability across sessions.

\section{A3. Calibration}

The intensities of the target and background lights were measured at the entrance pupil of the eye using a UDT radiometer, calibrated by the manufacturer (Gamma Scientific, San Diego, CA, USA) against a standard traceable to the US National Bureau of Standards. A spectroradiometer (EG\&G, San Diego, CA, USA) was used to measure spectral power distributions of all lights. Full methodological details can be found elsewhere (Stockman et al., 2007a; Stockman et al., 2007b; Stockman et al., 2008).

\section{Appendix B. Model of visual processing}

\section{B1. Normal model}

As discussed above, we model the early visual system as a sequence of stages, each stage behaving as a leaky integrator or low-pass (LP) filter. In order to account for normal data, we found we needed at least 6 LP-stages and two stages of subtractive inhibition (Rider et al., 2019). In this serial cascade the input of each stage is the output of the preceding stage.

The application of these "linear" systems in vision research has a long history (de Lange, 1958; Ives, 1922; Schade, 1956). One crucial property of such systems is that for sinusoidal inputs, such as those used in our experiments, the outputs are also sinusoidal and the only significant alterations from the sinusoidal input are changes in amplitude and delays. In general, the changes will depend on the frequency of the sinewave. If one stage reduces the amplitude by a factor of 2 , say, and the following stage reduces it by a factor of 3 , then the 
two stages in sequence will reduce the amplitude by a factor of 6 (i.e., the product of the individual reductions). The same reasoning applies to any length of cascade, and the combined response of all the stages will be equal to the product of the individual stages. If $A_{i}$ denotes the amplitude response of the $i^{\text {th }}$ stage, then the combined response for an $\mathrm{m}$ stage model, $A$ is,

$$
A_{N}=A_{1} \times A_{2} \times \ldots \times A_{m},
$$

where the subscript $\mathrm{N}$ denotes the observer has normal vision. For a single LP-stage, the amplitude response $A(f)$ at a given temporal frequency, $f$, is given by:

$$
A(f)=\frac{g}{2 \pi \sqrt{f^{2}+f_{c}^{2}}}
$$

where $f_{c}$ is the "corner frequency" and $g$ is a gain factor. The corner frequency is helpful in visualizing the filter's effect since it indicates the approximate frequency above which the filter starts to attenuate the response.

For a single stage of subtractive inhibition, the amplitude response, $A_{\text {inh }}(f)$, is:

$$
A_{i n h}(f)=\frac{\sqrt{f^{2}+\left((1-k) f_{c}\right)^{2}}}{\sqrt{f^{2}+f_{c}^{2}}}
$$

where $f_{c}$ is the corner frequency of the LP-stage in the inhibition and $k$ is the strength of the inhibition, such that $k=1$ produces complete inhibition at low frequencies and $k=0$ produces no inhibition.

Our normal model (illustrated in Figures 4 and 14) is the product of six LP-stages (based on our earlier work we assume four have corner frequencies that vary with light level and two have fixed corner frequencies) (Equation A2) and two stages of subtractive inhibition (Equation A3).

\section{B2. Abnormal model}

If we now assume that a genetic mutation causes a change in only one of these stages (to $B_{j}$, say, in the $\mathrm{j}^{\text {th }}$ ) then the response of a patient will be, 


$$
A_{p}=A_{1} \times \ldots A_{j-1} \times B_{j} \times A_{j+1} \times \ldots \times A_{m}
$$

where the subscript $P$ denotes a patient, $B_{j}$ is the response of the $j^{\text {th }}$ stage in the patient. By taking the ratio of the normal to the patient responses we see that the other $m-1$ stages cancel, and we are left with just the ratio of the stages that differ,

$$
\frac{A_{N}}{A_{p}}=\frac{A_{j}}{B_{j}}
$$

Here, we are mainly interested in the differences between normal observers $(N)$ and patients $(P)$. Assuming just one stage differs between normal observers and patients, Equation A2 shows that the ratio of responses for normal observers versus patients will be,

$$
\frac{A_{N}}{A_{P}}=\frac{G \sqrt{f^{2}+f_{c P}^{2}}}{\sqrt{f^{2}+f_{c N}^{2}}},
$$

where $G$ is the ratio of the normal to patient gain. For high frequencies where $f \gg{ }_{f c N}$ and and $f \gg>f_{c p}$, the response ratio tends toward $G$, while at low frequencies, where $f$ is much lower than both corner frequencies, the ratio tends to $G \frac{f_{c P}}{f_{c N}}$. Note that in fitting $A_{N} / A_{P}$ to the data we must estimate $G, f_{C P}$ and $f_{C N}$, allowing us to determine the speed of normal and abnormal stages.

Similarly, if two different stages differ from normal, then the ratio of the responses will be equal to the ratio of the differing stages,

$$
\frac{A_{N}}{A_{P}}=\frac{A_{j} A_{k}}{B_{j} B_{k}}=\frac{G \sqrt{f^{2}+f_{c P_{1}}^{2}} \sqrt{f^{2}+f_{c P_{2}}^{2}}}{\sqrt{f^{2}+f_{c N_{1}}^{2}} \sqrt{f^{2}+f_{c N_{2}}^{2}}} .
$$

Figure 5 in the main paper illustrates how the differences between normal and patient flicker sensitivities will change depending on the specific nature of the defect(s) in the patient cascade. The effect of one stage slowing down (equation A6 with $f_{C P}<f_{C N}$ ) or speeding up (equation $\mathrm{A} 6$ with $f_{c P}>f_{C N}$ ) is shown in panels $(\mathrm{A})$ and $(\mathrm{B})$ at the bottom of Figure 5. A general loss of sensitivity (equation $A 7$ with $f_{C P}=f_{C N}$ and $G>1$ ) is shown in panel 
(C). A loss of subtractive inhibition is shown in panel (D), which is the same form as panel (A) because Equation $\mathrm{A} 3$ is equivalent to equation $\mathrm{A} 6$ with $G=1$ and $f_{C P}=(1-k) f_{C N}<f_{C N}$.

\section{Appendix C. Normative TCSF data}

Tables $\mathrm{C} 1$ and $\mathrm{C} 2$ give the normative means and standard errors for the data shown in Figures 2 and 3, respectively.

\section{Table C1}

Mean flicker sensitivity data for 17 normal observers measured at the High mean 650-nm radiance. Column 1 gives the frequency of the sinusoidal flicker in hertz. Columns 2 and 3 give the logarithm of the amplitude sensitivity and two standard errors of the mean, respectively.

\begin{tabular}{|c|c|c|}
\hline $\begin{array}{c}\text { Frequency } \\
(\mathrm{Hz})\end{array}$ & $\begin{array}{c}\log _{10} \\
\text { amplitude } \\
\text { threshold }\end{array}$ & 2 S.E. \\
\hline 0.5 & -9.32 & 0.11 \\
1 & -9.11 & 0.08 \\
2 & -8.97 & 0.09 \\
4 & -8.74 & 0.07 \\
6 & -8.59 & 0.07 \\
8 & -8.53 & 0.05 \\
10 & -8.55 & 0.04 \\
15 & -8.74 & 0.03 \\
20 & -9.02 & 0.03 \\
25 & -9.27 & 0.04 \\
30 & -9.51 & 0.03 \\
35 & -9.81 & 0.04 \\
40 & -10.12 & 0.07 \\
\hline
\end{tabular}




\section{Table C2}

Mean flicker sensitivity data and standard errors for 8 normal observers measured at the Low (Columns 2 and 3), Medium-Low (Columns 4 and 5), Medium-High (Columns 6 and 7) and High (Columns 8 and 9) mean 650-nm radiance levels. Column 1 gives the frequency of the sinusoidal flicker in hertz. The data are tabulated as $\log _{10}$ amplitude sensitivities.

\begin{tabular}{|c|c|c|c|c|c|c|c|c|}
\hline \multirow[b]{2}{*}{$\begin{array}{l}\text { Frequency } \\
\qquad(\mathrm{Hz})\end{array}$} & \multicolumn{2}{|c|}{ Low level } & \multicolumn{2}{|c|}{$\begin{array}{l}\text { Medium-Low } \\
\text { level }\end{array}$} & \multicolumn{2}{|c|}{$\begin{array}{c}\text { Medium-High } \\
\text { level }\end{array}$} & \multicolumn{2}{|c|}{ High level } \\
\hline & $\begin{array}{l}\log _{10} \\
\text { amplitude } \\
\text { threshold }\end{array}$ & S.E. & $\begin{array}{c}\log _{10} \\
\text { amplitude } \\
\text { threshold }\end{array}$ & S.E. & $\begin{array}{c}\log _{10} \\
\text { amplitude } \\
\text { threshold }\end{array}$ & S.E. & $\begin{array}{l}\log _{10} \\
\text { amplitude } \\
\text { threshold }\end{array}$ & S.E. \\
\hline 0.5 & -6.21 & 0.07 & -6.75 & 0.08 & -7.80 & 0.06 & -9.04 & 0.08 \\
\hline 1 & -6.22 & 0.06 & -6.69 & 0.08 & -7.64 & 0.08 & -8.85 & 0.07 \\
\hline 2 & -6.26 & 0.06 & -6.66 & 0.09 & -7.51 & 0.07 & -8.73 & 0.08 \\
\hline 4 & -6.34 & 0.06 & -6.63 & 0.07 & -7.34 & 0.07 & -8.47 & 0.07 \\
\hline 6 & -6.41 & 0.04 & -6.70 & 0.05 & -7.29 & 0.05 & -8.33 & 0.09 \\
\hline 8 & -6.52 & 0.04 & -6.73 & 0.04 & -7.34 & 0.06 & -8.29 & 0.08 \\
\hline 10 & -6.66 & 0.05 & -6.88 & 0.05 & -7.42 & 0.07 & -8.34 & 0.08 \\
\hline 15 & -7.05 & 0.05 & -7.23 & 0.06 & -7.67 & 0.06 & -8.51 & 0.05 \\
\hline 20 & & & -7.66 & 0.06 & -8.00 & 0.05 & -8.81 & 0.04 \\
\hline 25 & & & -8.04 & 0.06 & -8.32 & 0.04 & -9.12 & 0.04 \\
\hline 30 & & & & & -8.67 & 0.06 & -9.34 & 0.05 \\
\hline 35 & & & & & & & -9.58 & 0.07 \\
\hline 40 & & & & & & & -9.81 & 0.06 \\
\hline
\end{tabular}

\section{Appendix D. New model fits}

We have extensively reanalysed many of our earlier measurements using the updated version of the model described in the main text. The fits were carried out using the standard non-linear fitting Marquardt-Levenberg algorithm (Levenberg, 1944; Marquardt, 1963) implemented in SigmaPlot (Systat Software, San Jose, CA) to minimize the sum of the squared differences between the data and model predictions. The model fits were made 
using logarithmic forms of the equations given in Appendix B and the best-fitting parameters are given with \pm 1 standard error of the fitted parameter. More details of the fits can be found in the original papers.

In some fits (see below), the frequency constant was limited to a maximum of $100 \mathrm{~Hz}$. Above that corner frequency, the filter is essentially equivalent to a frequency-independent gain adjustment over the measured frequency range (of up to only $30 \mathrm{~Hz}$ or less in patients), with the result that the frequency constant and gain parameters in the fit become unstable. Limiting the corner frequency to values lower than $100 \mathrm{~Hz}$ has relatively little effect on the other fitted parameters until the corner frequency approaches the upper limit of the measured range of frequencies. The relative sensitivity loss for a single LP stage is $0.15 \log _{10}$ unit at the corner frequency and $0.1 \log _{10}$ unit at half the corner frequency (see Eqn A2). We set the upper limit to $100 \mathrm{~Hz}$, which reduces sensitivity at $30 \mathrm{~Hz}$ by only about $0.02 \log _{10}$ unit.

\section{D1. Rod achromatopsia (GNAT2) data}

We modelled the sensitivity losses for the father and son with rod achromatopsia measured by Stockman et al. (2007b). The losses for both patients can be accounted for simply by the addition of two low-pass stages defined by Equation (A2) with a corner frequency of $f_{c P}(\mathrm{~Hz})$ and a logarithmic gain adjustment of $\log _{10}(g)$. The best-fitting model parameters \pm 1 standard error of the fitted parameter and the adjusted $R^{2}$ and the standard error of the estimate (SEE) are given in Table D1.

\section{Table D1}

\begin{tabular}{l|c|c|c|}
\hline Observer & $f_{C P}(\mathrm{~Hz})$ & $\log _{10}(g)$ & $R^{2} / \mathrm{SEE}$ \\
\hline Father & $1.01 \pm 0.09$ & $1.59 \pm 0.04$ & $0.993 / 0.05$ \\
Son & $1.07 \pm 0.35$ & $1.00 \pm 0.16$ & $0.823 / 0.12$
\end{tabular}

Note that because these filters are so much slower than the normal filters they replace in the normal sequence, we can ignore the normal filters (i.e., we can use Equation (A2) rather than (A7) for the model fits). The model here is applied to the losses of the patients relative to normals, whereas in the original paper it was applied to the patient flicker sensitivities. 
Because of the limited frequency range, we cannot determine if other stages speed up to compensate for the slowed stages rather than changing the gain.

\section{D2. Bradyopsia data}

We modelled the sensitivity losses for the single Bradyopsia patient measured by Stockman et al. (2008). To account for the data, we altered the corner frequencies of two LP-stages in the normal model by fitting Equation (A7). The two LP-stages have the same corner frequencies for normals $\left(f_{C N 1}=f_{C N 2}\right)$, and we slowed down one to a corner frequency of $f_{C P 1}$ in the patient and sped up the other to a corner frequency of $f_{C P 2}$ in the patient, and added a logarithmic gain adjustment of $\log _{10}(G)$. The model was fitted simultaneously across the four mean intensity levels. The best-fitting parameters, $R^{2}$ and SEE are given in the far column of Table D2. For simplicity, $f_{C N 1}$ and $f_{C N 2}$ were assumed to be the same at each level and $f_{C P 2}$ was limited to be $\leq 100 \mathrm{~Hz}$. The limit was imposed because changes in corner frequency above $100 \mathrm{~Hz}$ have roughly the same effect over the visible range of frequencies as varying $\log _{10}(\mathrm{G})$, so that allowing $f_{C P 2}$ to vary above $100 \mathrm{~Hz}$ made the fits unstable.

\section{Table D2}

\begin{tabular}{|c|c|c|c|c|c|}
\hline Level & $f_{C N 1}, f_{C N 2}(\mathrm{~Hz})$ & $f_{C P 1}(\mathrm{~Hz})$ & $f_{C P 2}(\mathrm{~Hz})$ & $\log _{10}(G)$ & $R^{2} / \mathrm{SEE}$ \\
\hline Low & $7.38 \pm 1.27$ & $1.93 \pm 0.56$ & $100 *$ & $0.16 \pm 0.07$ & \multirow{4}{*}{$\begin{array}{c}0.957 / \\
0.06\end{array}$} \\
\hline $\begin{array}{l}\text { Medium- } \\
\text { Low }\end{array}$ & $9.05 \pm 1.90$ & $3.79 \pm 1.31$ & $100 *$ & $-0.16 \pm 0.07$ & \\
\hline $\begin{array}{l}\text { Medium- } \\
\text { High }\end{array}$ & $15.76 \pm 1.74$ & $1.21 \pm 0.24$ & $100 *$ & $-0.78 \pm 0.07$ & \\
\hline High & $11.92 \pm 1.01$ & $0.98 \pm 0.21$ & $100 *$ & $-0.68 \pm 0.04$ & \\
\hline
\end{tabular}

\section{D3. Dominant progressive cone dystrophy data}

We modelled the sensitivity losses for four patients from the same family with dominant progressive cone dystrophy measured at three intensity levels by Stockman et al. (2014b). To account for these data, we again modified two stages common to the normals and patients (Equation (A7) with $f_{C N 1}=f_{C N 2}$ ), one of which speeds up from a corner frequency of 
$f_{C N 1}$ in normals to one of $f_{C P 1}$ in the patients, and the other stage slows down from a corner frequency of $f_{C N 2}$ in normals to one of $f_{C P 2}$ in the patients, with a logarithmic gain adjustment of $\log _{10}(G)$. In one patient (the youngest, GP1) there was little evidence of slowing down at the Medium-High and High levels and the slow stage in the fit was limited to the speed of the normal stage by constraining $f_{C P 2} \geq f_{C N 2}$. For simplicity, $f_{C N 1}$ and $f_{C N 2}$ were assumed to be the same and ${ }^{*} f_{C P 1}$ was limited to be $\leq 100 \mathrm{~Hz}$. The model was fitted simultaneously across the four patients at each level. The best-fitting parameters are given in rows 1-12 of Table D3. The model is like the model used by Stockman et al. (2014b).

Table D3

\begin{tabular}{|c|c|c|c|c|c|c|}
\hline Level & Patient & $f_{C N 1}, f_{C N 1}(\mathrm{~Hz})$ & $f_{C P 1}(\mathrm{~Hz})$ & $f_{C P 2}(\mathrm{~Hz})$ & $\log _{10}(G)$ & $R^{2} / \mathrm{SEE}$ \\
\hline \multirow{5}{*}{$\begin{array}{l}\text { Medium- } \\
\text { Low }\end{array}$} & GP1 & \multirow{5}{*}{$7.72 \pm 2.00$} & \multirow{5}{*}{$100^{*}$} & $6.68 \pm 3.69$ & $-0.10 \pm 0.07$ & \multirow{5}{*}{$\begin{array}{c}0.885 / \\
0.11\end{array}$} \\
\hline & GP2 & & & $2.54 \pm 1.20$ & $0.03 \pm 0.09$ & \\
\hline & & & & & & \\
\hline & GP3 & & & $0.92 \pm 0.42$ & $-0.89 \pm 0.21$ & \\
\hline & GP4 & & & $1.30 \pm 0.51$ & $-0.72 \pm 0.18$ & \\
\hline \multirow{4}{*}{$\begin{array}{l}\text { Medium- } \\
\text { High }\end{array}$} & GP1 & \multirow{4}{*}{$12.51 \pm 2.17$} & \multirow{4}{*}{$100^{*}$} & $12.51^{\#}$ & $-0.01 \pm 0.07$ & \multirow{4}{*}{$\begin{array}{c}0.915 / \\
0.11\end{array}$} \\
\hline & GP2 & & & $2.24 \pm 0.68$ & $-0.37 \pm 0.10$ & \\
\hline & GP3 & & & $0.48 \pm 0.29$ & $-0.94 \pm 0.12$ & \\
\hline & GP4 & & & $0.98 \pm 0.35$ & $-1.07 \pm 0.13$ & \\
\hline \multirow[t]{4}{*}{ High } & GP1 & \multirow{4}{*}{$33.20 \pm 10.13$} & \multirow{4}{*}{$100 *$} & $33.20^{\#}$ & $-0.42 \pm 0.16$ & \multirow{4}{*}{$\begin{array}{c}0.924 / \\
0.09\end{array}$} \\
\hline & GP2 & & & $4.11 \pm 0.98$ & $-0.93 \pm 0.22$ & \\
\hline & GP3 & & & $1.22 \pm 0.32$ & $-1.43 \pm 0.24$ & \\
\hline & GP4 & & & $1.14 \pm 0.30$ & $-1.66 \pm 0.25$ & \\
\hline
\end{tabular}

* $f_{C P 2}$ was limited to $100 \mathrm{~Hz}$

${ }^{\#} f_{C P 2}$ was constrained to be $\geq f_{C N 1,2}$ 


\section{D4. LCA2 data}

We reanalysed and modelled the mean sensitivity losses for 5 LCA2 patients measured by Ripamonti et al. (2014b). We allowed two stages to slow down from the same corner frequencies of $f_{C N}$ in the normal to ones of $f_{C P}$ in the patient and allowed a logarithmic gain adjustment of $\log _{10}(G)$, i.e., Equation (A7) with $f_{C N 1}=f_{C N 2}=f_{C N}$, and $f_{C P 1}=f_{C P 2}=f_{C P}$ (see Table D4).

Table D4

\begin{tabular}{|c|c|c|c|}
\hline$f_{C N}(\mathrm{~Hz})$ & $f_{C P}(\mathrm{~Hz})$ & $\log _{10}(G)$ & $R^{2} / \mathrm{SEE}$ \\
\hline $20.79 \pm 4.14$ & $2.29 \pm 0.14$ & $-1.82 \pm 0.15$ & $\begin{array}{c}0.996 / \\
0.15\end{array}$
\end{tabular}

\section{D5. DOA data}

We reanalysed and modelled the mean sensitivity losses for the 11 DOA patients measured by Majander et al. (2017). For the mean patient losses, we allowed one stage to slow down from a corner frequency of $f_{C N 1}$ in the normal to one of $f_{C P 1}$ in the patient and another stage to speed up from a corner frequency of $f_{C N 2}$ in the normal to one of $f_{C P 2}$ in the patient and allowed a logarithmic gain adjustment of $\log _{10}(G)$ (Equation (A7) with $f_{C N 1}=f_{C N 2}$, see Table D5, row 1). $f_{c P 2}$ was limited to $100 \mathrm{~Hz}$ because higher values cause minimal effects on the shapes of the sensitivity loss function in the visible range of frequencies. We also separately fitted the mean losses for younger ( $<40$ years old) and older ( $>40$ years old) patients (Equation (A7) with $f_{C N 1}=f_{C N 2}$ ). For the younger patients, we used the same fit as for the mean losses for all patients and allowed a stage that slows down $\left(f_{C N 1}\right.$ to $\left.f_{C P 1}\right)$ and another that speeds up $\left(f_{C N 2}\right.$ to $\left.f_{C P 2}\right)$ and is limited to $100 \mathrm{~Hz}$ (see Table D5, row 2). For the older patients, we allowed a single stage that slows down $\left(f_{C N 1}\right.$ to $\left.f_{C P 1}\right)$ and another stage that could speed up but was constrained to be less than or equal to the normal speed $\left(f_{C N 2} \geq f_{C P 2}\right)$ (see Table D5, row 3). In the final fit we found that the "faster" stage was not faster than normal, so the fit here is effectively one LP-stage slowing down, i.e., Equation (A6), rather than two stages changing, i.e., Equation (A7), as for the older patients. 


\section{Table D5}

\begin{tabular}{cccccccc|}
\hline Ages & $f_{C N 1, \mathrm{CN} 2}(\mathrm{~Hz})$ & $f_{C P 1}(\mathrm{~Hz})$ & $f_{C P 2}(\mathrm{~Hz})$ & $\log _{10}(G)$ & $R^{2} / \mathrm{SEE}$ \\
\hline All & $14.71 \pm 1.55$ & $3.55 \pm 0.55$ & $100^{*}$ & $0.00 \pm 0.05$ & $\begin{array}{c}0.891 / \\
0.20\end{array}$ \\
\hline $13-19$ & & $3.93 \pm 1.26$ & $100^{*}$ & $0.13 \pm 0.07$ & $0.850 /$ \\
$47-54$ & $12.52 \pm 2.45$ & $3.21 \pm 0.78$ & $12.52^{\#}$ & $-0.75 \pm 0.07$ & 0.20
\end{tabular}

* $f_{C P 2}$ was constrained to be $<100 \mathrm{~Hz}$

${ }^{\#} f_{C P 2}$ was constrained to be $\geq f_{C N 1,2}$

Note that although we interpret these losses as if they were due mainly to a stage slowing down, i.e., (A) in Figure 5, an alternative interpretation, which might be consistent with the loss of ganglion cells, is that they are due to a reduction in the strength of feedforward inhibition, i.e., (D) in Figure 5. This would produce identical changes in the frequency response (see section B of Appendix).

\section{D6. Supernormal rod ERG data}

We reanalysed and modelled the mean sensitivity losses for observers with cone dystrophy with supernormal rod ERG (KCNV2) from Stockman et al. (2014a) again using Equation (A7) with $f_{C N 1}=f_{C N 2}$. We allowed one stage to slow down from a corner frequency of $f_{C N 1}$ in the normal to one of $f_{C P 1}$ in the patient and another stage to speed up from a corner frequency of $f_{C N 2}$ in the normal to one of $f_{C P 2}$ in the patient with a logarithmic gain adjustment of $\log _{10}(G)$ (see Table D6, row 2). The parameter $f_{C P 2}$ was given a minimum limit equal to the normal speed $\left(f_{C P 2} \geq f_{C N 2}\right)$ and an upper limit of $100 \mathrm{~Hz}$ because higher values cause minimal effects on the shapes of the sensitivity loss function in the visible range of frequencies. For patient SR5 the "faster" stage converged to the normal speed, so their data was effectively fitted by a single LP-stage slowing, Equation (A6), rather than two stages changing, Equation (A7).

\section{Table D6}

Patient $f_{C N 1, c N 2}(\mathrm{~Hz}) \quad f_{C P 1}(\mathrm{~Hz})$ $f_{C P 2}(\mathrm{~Hz})$ $\log _{10}(G)$ $R^{2} / \mathrm{SEE}$ 


\begin{tabular}{ccccc}
\hline SR1 & $3.02 \pm 0.73$ & $100^{*}$ & $-0.04 \pm 0.04$ & \\
SR2 & & & & \\
SR3 & $9.90 \pm 0.30$ & $10.64 \pm 3.98$ & $-0.99 \pm 0.08$ & \\
SR4 & $2.68 \pm 0.64$ & $100^{*}$ & $-0.34 \pm 0.05$ & $0.940 /$ \\
& & & & 0.08 \\
SR5 & $3.06 \pm 0.74$ & $100^{*}$ & $-0.05 \pm 0.04$ & \\
as limited to $100 \mathrm{~Hz}$ & $2.54 \pm 0.81$ & $9.32 \pm 3.57^{\#}$ & $-0.37 \pm 0.06$ & \\
nnstrained to be $\geq f_{C N 2}$ & & & & \\
\end{tabular}

$* f_{C P 2}$ was limited to $100 \mathrm{~Hz}$
$\# f_{C P 2}$ constrained to be $\geq f_{C N 2}$

D7. Enhanced S-cone syndrome data

L-cone data from Ripamonti et al. (2014a). The flicker sensitivity data for ECS1 were not published in the original paper.

\section{FIGURE LEGENDS}

\section{Figure 1.}

Simplified diagrams of the retinal locations affected by known gene defects in the seven eye diseases for which we have flicker-sensitivity data. The diseases, labelled (A-G), and the five associated defects that affect the photoreceptors are listed in the yellow panel. Three of the defects are in molecules in the transduction cascade within the photoreceptor outer segment (upper right-hand diagram): Defects in GNAT2, (A), which encodes cone $\alpha$ transducin, disrupt the cascade; defects in RGS9-1, (B), which encodes a GTPase accelerating protein, slow down the shutting off of the visual response; and defects in GUCA1A (GCAP1), (C), which encodes a guanylate cyclase activating protein that restores CGMP, speed up the shutting off the visual response. Beyond the outersegment (left diagram), defects in RPE65, (D), which encodes a retinal pigment epithelium-isomerase, upset the regeneration of bleached visual pigment; defects in KCNV2, (F), which encodes a voltage-gated potassium channel, distort the visual response; and defects in NR3E2, (G), which encodes a photoreceptor-specific nuclear receptor, disrupt the numbers of rods and cones produced 
during development leading to an excess of S-cones. After the photoreceptors, defects in OPA1, (E), which encodes an inner mitochondrial membrane protein, lead to the loss of ganglion cells. For more details, see text.

\section{Figure 2.}

Normative flicker sensitivity data. (Panel A): The panel shows flicker sensitivities for 17 observers with normal vision (see key). The logarithm of amplitude sensitivity (the logarithm of the reciprocal of amplitude threshold in quanta $\mathrm{s}^{-1} \mathrm{deg}^{-2}$ ) is plotted as a function of frequency $(\mathrm{Hz})$. The error bars are \pm 1 S.E.M. of three or more separate flicker measurements. The grey straight lines are individually fitted to the high-frequency region of each flicker sensitivity function. (Panel B): The orange line shows the mean flicker sensitivities and the dashed white lines indicate \pm 2 S.E.M. across observers. The flicker sensitivities for the 17 observers have been shifted vertically to align their high-frequency regions (between 15 and $40 \mathrm{~Hz}$ ) with the mean curve (using a minimum least-squares criterion); vertical shifts on the log scale are equivalent to multiplying an observer's sensitivity by a constant, in order to match the high-frequency sensitivity of the average observer. The brown line is fitted to the high-frequency end of the aligned data and the frequency constant associated with the line is $19.19 \pm 0.42 \mathrm{~Hz}\left(R^{2}=0.997\right)$. The mean $650-\mathrm{nm}$ target radiance was $10.38 \log _{10}$ quanta s $\mathrm{s}^{-1}$ $\operatorname{deg}^{-2}\left(3.24 \log _{10}\right.$ photopic troland)-the High level in the text. The 481-nm background radiance was $8.20 \log _{10}$ quanta $\mathrm{s}^{-1} \mathrm{deg}^{-2}\left(1.31 \log _{10}\right.$ photopic trolands). (Panel C): Illustration of the technique for measuring flicker sensitivity. The observer adjusts the amplitude of a sinusoidally-flickering light of fixed frequency to find the smallest amplitude at which flicker can just be seen. The time-averaged mean intensity, shown by the dashed horizontal lines at each flicker amplitude, is kept constant.

\section{Figure 3.}

Normative mean flicker sensitivities \pm 1 S.E.M. averaged over eight observers with normal vision measured at the four standard intensities: the Low (inverted brown triangles), Medium-Low (red squares), Medium-High (orange triangles) and High (yellow circles) adaptation levels. The best fitting straight lines to the high-frequency end of each sensitivity 
function have frequency constants of $13.90 \pm 0.68$ (Low, brown line, $R^{2}=0.993$ ), $13.60 \pm 0.59$ (Medium-Low, red line, $R^{2}=0.991$ ), $16.48 \pm 0.55$ (Medium-High, orange line, $R^{2}=0.994$ ) and $19.80 \pm 0.57$ (High, yellow line, $R^{2}=0.995$ ) $\mathrm{Hz}$. For all measurements, the 481-nm background radiance was $8.20 \log _{10}$ quanta $\mathrm{s}^{-1} \mathrm{deg}^{-2}$.

\section{Figure 4.}

Sequential model of visual processing in normal observers. The essential components of the model are a gain control ( $g$ ), 6 low-pass (LP) stages ([1]-[6]), and 2 feed-forward stages, each of which is made up of a separate LP-stage, a gain control $(k)$ and a signal inversion (-). The order of these components is not constrained in the model, so that the ordering in the figure is essentially arbitrary (but see Figure 14). The six LP stages [1]-[6] in the square boxes on the central black line are in the direct pathway, and two LP stages $[A]$ and $[B]$ are in feedforward loops that provide subtractive inhibition. The signal level can be adjusted at gain controls at three locations: one labelled $g$ controls the direct pathway; and two labelled $k$ control the amount of feedforward inhibition. Signal inversions (-) highlighted in blue circles make the feedforward inhibitory.

Probes (1)-(4) trace the effects of the stages on an input signal as it passes through the sequence. The input signal (panel 1 on the left) is a brief pulse of light. The input signal and the successive responses to that signal are plotted as a function of time in the left-hand panels. The corresponding amplitude response as a function of frequency is shown in the green panels on the right (mathematically the two columns derive from Fourier transform pairs, the phase response is not shown).

In this illustration, the corner frequencies that determine the characteristics of the LPstages ([1]-[4], [A], [B]) are all $15 \mathrm{~Hz}$, while those of the two late LP-stages ([5], [6]) are 30 $\mathrm{Hz}$. The feedback gain, $k$, is 0.8 . The overall gain, $g$, is arbitrary, since, for clarity, the graphs of the responses have all been normalised to peak at one. (See text and Rider, Henning \& Stockman (2019) for more details.) 


\section{Figure 5.}

Illustrative differences between the responses of a normal processing sequence on the left and, on the right, the same sequence with one abnormality that slows down the response at Stage [2] (highlighted in red). The input signals and the responses are shown as a function of frequency. The isolated response of the normal second stage is shown in the second green panel, and the isolated response of the abnormal stage is shown in the second (red) panel. At the bottom of the left-hand column is the output response of the normal sequence (bottom green panel) to the amplitude spectrum of an input pulse (top green panel), and at the bottom of the right-hand column is the output response of the abnormal sequence to the same input pulse (top panel). The middle column shows the differences between the normal and abnormal responses plotted as the logarithm of the ratio of the abnormal to the normal response. (Zero log difference corresponds to a ratio of 1, i.e., identical responses.) At the input, there is no difference (white panel). Immediately after the second stage, the differences are simply the differences between the normal and abnormal responses at that stage (yellow panel, labelled $(\mathbf{A})$ ). The final differences in the outputs of the two systems are shown in the bottom orange panel. The final differences reflect not only the difference of the directly affected stage propagated to the output but also any additional differences because of compensatory adjustments in the stages in the abnormal system that follow the abnormal stage ([3]-[6], [A], [B], highlighted in orange in the righthand sequence). In this example, the time-averaged and low frequency signals after the abnormality at [2] will be larger than normal, and, as a result, the later stages may speed up and/or alter their gain because of light adaptation.

At the bottom of the figure the yellow panels in the cyan box show four examples of the output differences that might be expected from four different types of abnormality. (Panel A) shows the effect of an abnormal stage slowing down (as in the second, central panel of the upper figure); (Panel B) shows the effect of an abnormal stage speeding up; (Panel C) shows the effect of a gain change that decreases the response equally at all frequencies; and (Panel D) shows the effect of a loss of surround inhibition. See text for details.

\section{Figure 6.}


Rod achromatopsia (GNAT2). Data for the affected father are shown by red triangles and for the son by green inverted triangles. (Panel A): Flicker sensitivity data \pm 1 S.E.M. (across three separate measurements) for the patients and the mean data for the normal observers over the patients' restricted range (orange line) \pm 2 S.E.M. (across 17 observers, dashed white lines). The lines fitted to the high frequency ends of the flicker sensitivity functions have frequency constants of $7.10 \pm 0.25$ (red line, $R^{2}=0.994$ ) and $6.40 \pm 1.11$ (green line, $R^{2}=0.916$ ) Hz. (Panel B): Sensitivity losses for the patients (coloured symbols) relative to normal sensitivities. Best-fitting versions of model for the abnormality has two extra LP-stages each defined by Equation (A2) with corner frequencies of $1.01 \mathrm{~Hz}$ for the father (red line) and $1.07 \mathrm{~Hz}$ for the son. See text and Appendix D1 for further details. Patient data from Stockman et al. (2007b).

\section{Figure 7.}

Bradyopsia (RGS9) (Panel A): Flicker sensitivities \pm 1 S.E.M. for one patient with Bradyopsia at the Low, Medium-Low, Medium-High and High intensity levels (brown, red, orange and yellow triangles, respectively). The frequency constants are $15.59 \pm 1.16$ (brown line, $R^{2}=0.968$ ), $18.31 \pm 0.58$ (red line, $R^{2}=0.994$ ), $16.77 \pm 0.39$ (orange line, $R^{2}=0.996$ ), and 27.14 \pm 1.67 (yellow line, $R^{2}=0.971$ ) $\mathrm{Hz}$. For comparison, the mean normative data from Figure 3 are replotted as coloured circles using the same colour code for the intensity levels. The best fitting lines that define the mean normal frequency constants are shown by the white dashed lines. The error bars are \pm 1 S.E.M. across observers. (Panel B): Logarithmic sensitivity losses for the patient at the Low, Medium-Low, Medium-High and High levels (symbols as panel A) relative to the normal sensitivities. The lines are best-fitting versions of the model in which one of two low-pass stages common to normal observers and to the Bradyopsia patient slows down in the patient and the other speeds up, Equation (A7). See text and Appendix D2 for further details. Patient data from Stockman et al. (2008). The number in brackets in the legend denotes age at testing. 


\section{Figure 8.}

Dominant, progressive cone dystrophy (GUCA1A). (A): Flicker sensitivities for GP1 (triangles), GP2 (circles), GP3 (inverted triangles) and GP4 (diamonds) at the Medium-Low (red symbols and lines), Medium-High (orange symbols and lines) and High (yellow symbols and lines) levels. The frequency constants are for GP1 33.00 \pm 4.06 (red line, $R^{2}=0.928$ ), $7.83 \pm 1.84$ (orange line, $R^{2}=0.974$ ) and 27.33 \pm 1.25 (yellow line, $R^{2}=0.988$ ); for GP2 18.85 \pm 1.53 (red line, $R^{2}=0.956$ ), $13.33 \pm 1.14$ (orange line, $R^{2}=0.964$ ) and $14.25 \pm 0.74$ (yellow line, $R^{2}=0.987$ ); for GP3: $10.41 \pm 2.62$ (red line, $R^{2}=0.831$ ), $29.74 \pm 1.67$ (orange line, $R^{2}=0.981$ ) and $19.68 \pm 0.93$ (yellow line, $R^{2}=0.989$ ); and for GP4 10.59 \pm 1.35 (red line, $R^{2}=0.953$ ), $18.14 \pm 1.00$ (orange line, $R^{2}=0.985$ ) and $12.12 \pm 0.83$ (yellow line, $R^{2}=0.982$ ). (B): Sensitivity losses at the Medium-Low, MediumHigh and High levels (left, middle, and right graphs, respectively) for GP1 (green triangles), GP2 (purple circles), GP3 (inverted yellow triangles), and GP4 (blue diamonds). The solid lines are best-fitting versions of the model in which one low-pass stage common to normal observers and patients speeds up in all patients and another stage slows down, Equation (A7) (or remains at the same speed for GP1 at Medium-High and High levels, Equation (A6)). See text and Appendix D3 for further details. Patient data from Stockman et al. (2014b). The numbers in brackets in the legend denote age at testing.

\section{Figure 9.}

Leber's congenital amaurosis (RPE65). (Panel A): Flicker sensitivity data \pm 1 S.E.M for five patients: S1 (green diamonds), S3 (dark-blue triangles), S5 (red circles), S6 (purple hexagons) and S12 (blue squares) and the mean normal data \pm 2 S.E.M. (solid orange and dashed white lines). The frequency constants are for S1: 9.75 \pm 0.59 (green line, $R^{2}=0.985$ ), S2: $13.63 \pm 0.92$ (dark blue line, $R^{2}=0.973$ ), S5: $9.83 \pm 0.35$ (red line, $R^{2}=0.994$ ), S6: $10.24 \pm 0.73$ (purple line, $\left.R^{2}=0.975\right)$ and S12: $16.94 \pm 1.37$ (blue line, $\left.R^{2}=0.962\right) \mathrm{Hz}$. (Panel B): Logarithmic sensitivity losses for the five patients (coloured symbols) relative to the normal observer. The data from the patients have been vertically shifted to align with the mean patient losses \pm 1 S.E.M. (dotted yellow squares in Panel C). Relative to the mean losses, the individual losses for each patient have been shifted in $\log _{10}$ units by -0.57 for S1, -0.14 for S3, 0.35 for S5, 0.02 for S6 and 0.13 
for S12. The mean losses can be accounted for by slowing down two stages in the normal observer (solid red line), Equation (A7). See text and Appendix D4 for further details. The patient data are from Ripamonti et al. (2014b). Data were measured separately in each eye and are shown averaged across eyes. Numbers in brackets in the legend denote age at testing.

\section{Figure 10.}

Dominant optical atrophy (OPA1). (Panel A): Flicker sensitivity data for the 11 patients \pm 1 S.E.M (coloured symbols, see key) with best-fitting high frequency slopes (solid and dashed grey lines) and mean normal data (orange and dotted white lines). The frequency constant for the mean patient data is $23.27 \pm 0.83 \mathrm{~Hz}$ (red-black dashed line, $\left.R^{2}=0.995\right)$. (Panel B): Logarithmic sensitivity losses for each patient (coloured symbols) relative to the normal sensitivities and the mean patient losses \pm 1 S.E.M. (yellow dotted squares). The dashed redblack line is the best-fitting version of a model in which one stage slows down in the patient and another speeds up, Equation (A7). (Panel C): Logarithmic sensitivity losses for the means \pm 1 S.E.M. of two age-groups of patients, $13-39$ years (white diamonds) and 47-54 years (grey hexagons) relative to the normal sensitivities. A simultaneous fit of two models are fitted to the two patient groups. One normal stage slows down in all patients and another normal stage speeds up in the younger patients (white line), Equation (A7), but not in the older patients (grey line), Equation (A6). See text and Appendix D5 for further details. Patient data from Majander et al. (2017). Numbers in brackets in the legend above (B) and (C) denote age at testing.

\section{Figure 11.}

Cone dystrophy with supernormal rod ERG (KCNV2). (Panel A): Flicker sensitivity data \pm 1 S.E.M for five patients SR1 (blue triangles), SR2 (pink inverted triangles), SR3 (green diamonds), SR4 (dark blue circles) and SR5 (brown hexagons) and the mean normal data \pm 2 S.E.M. (solid orange and dashed white lines). The frequency constants are for SR1: 36.10 \pm 4.86 (blue line, $R^{2}=0.900$ ), SR2: $21.36 \pm 3.17$ (pink line, $R^{2}=0.917$ ), SR3: $46.92 \pm 5.96$ (green line, $R^{2}=0.924$ ), SR4: $28.97 \pm 2.96$ (dark blue line, $R^{2}=0.950$ ) and SR5: $17.30 \pm 1.50$ (brown line, 
$\left.R^{2}=0.964\right) \mathrm{Hz}$. The frequency constant for the mean patient data is $27.42 \pm 1.66 \mathrm{~Hz}$ (red-black dashed line, $\left.R^{2}=0.987\right)$. Numbers in brackets in the legend denote age at testing. (Panel B): Logarithmic sensitivity losses for the five patients (coloured symbols) and the mean patient losses \pm 1 S.E.M. (dotted yellow squares) relative to the normal mean data. The standard model is fitted to the individual losses, Equation (A7) with two identical low-pass stages in the normal one of which speeds up and one slows down in the patients. As in other fits above, the faster stage is constrained below to be at least as fast as the normal stages, and above by $100 \mathrm{~Hz}$. See text and Appendix D6 for further details. Patient data from Stockman et al. (2014a).

\section{Figure 12.}

Enhanced S-cone syndrome. Flicker sensitivities \pm 1 S.E.M for ESC1 (blue circles) and the mean normal data \pm 2 S.E.M. (solid orange and dashed white lines). The patient's high frequency constant is $18.58 \pm 1.02 \mathrm{~Hz}$ (blue line, $\left.R^{2}=0.985\right)$. Patient data from Ripamonti et al. (2014a).

\section{Figure 13.}

Frequency constants $(\mathrm{Hz})$ for patients separated by gene defect: GNAT2 (cyan circles), RGS9 (black square), GUCA1A (green and dotted green triangles), RPE65 (purple hexagons), and NR2E3 (blue square) and those for normal observers (yellow circles, with the mean normal frequency constant \pm 2 S.E.M. indicated by the horizontal yellow and dashed white lines). The OPA1 patients aged 13-39 and 47-54 are shown separately as grey and white diamonds, respectively. KCNV2 patients SR1, SR3 and SR4, who according to the model have a stage that speeds, are shown as orange inverted dotted triangles and SR2 and SR5, who do not, are shown as red inverted triangles. The dotted green triangle highlights GUCA1A patient GP1. As indicated by the arrow above the figure, one OPA1 patient has a frequency constant of $104 \mathrm{~Hz}$ that plots well above the graph panel. 


\section{Figure 14.}

The left diagram shows an equivalent version of the sequential model shown in Figure 4 rearranged for consistency with the human retinal physiology schematized in the right diagram. Details as Figure 4. The pink arrows and numbers correspond to the similarly labelled probes in Figure 4 and thus also to the corresponding panels of Figure 4 showing the input signal and sequential responses to the input signal. The right-hand diagram is part of Figure 3.5 from Stockman \& Brainard (2015) and shows, for comparison, (from top to bottom) cone photoreceptors, an $\mathrm{H} 1$ horizontal cell, diffuse ON (white) and OFF (grey) bipolar cells and an ON (white) and an OFF (grey) parasol ganglion cells.

\section{Figure A1.}

Simplified version of a 2-channel Maxwellian-view system showing also the observer's view. Two light sources are separately collimated and each illuminate one channel. A circular aperture in each channel creates the $4^{\circ}$ diameter target disc and the $9^{\circ}$ diameter background disc that are imaged on the retina. The beamsplitter superimposes and combines the beams. Finally, the Maxwellian lens and the optics of the eye image the lightfilled apertures onto the retina. 
FIGURE 1

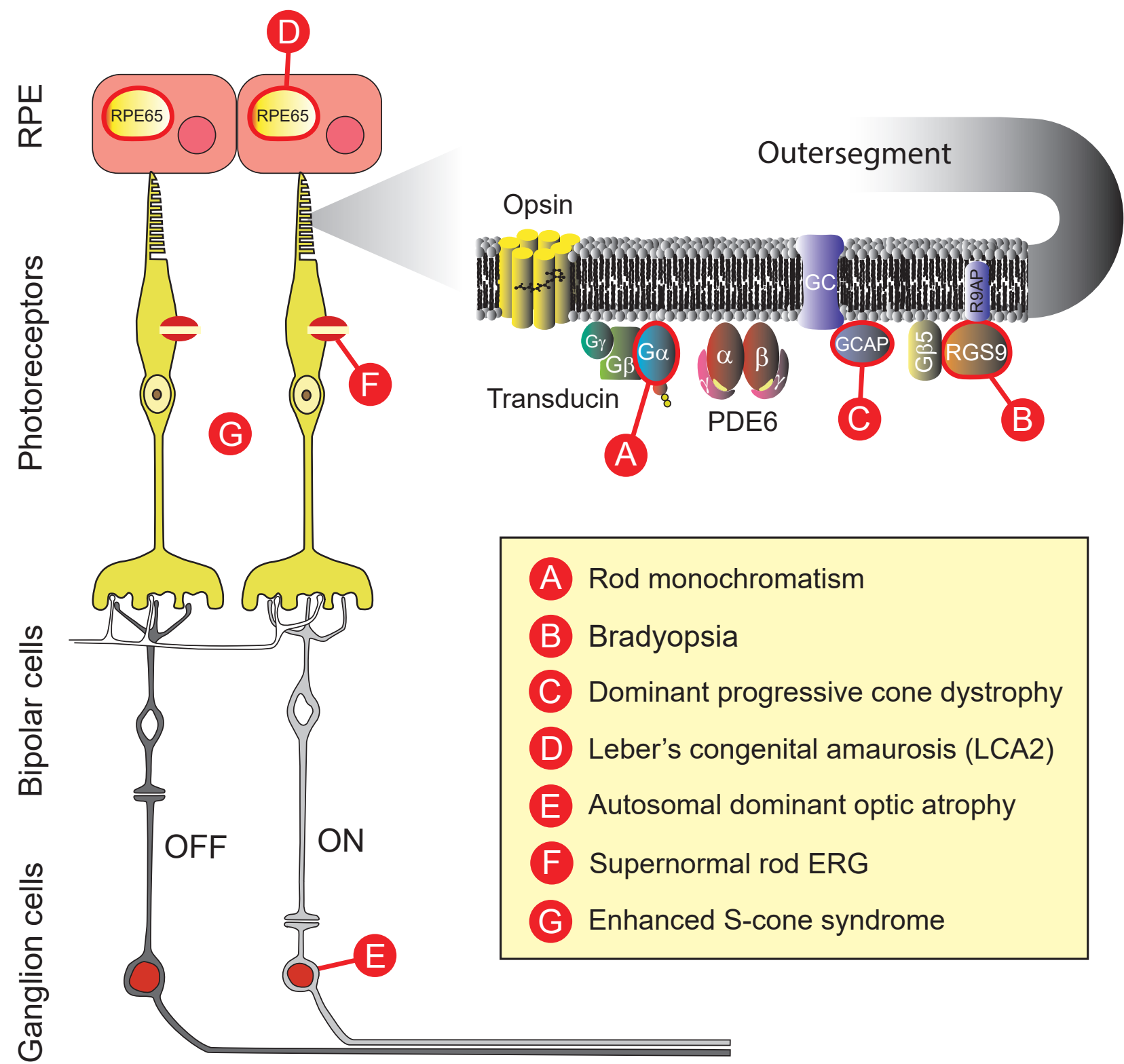




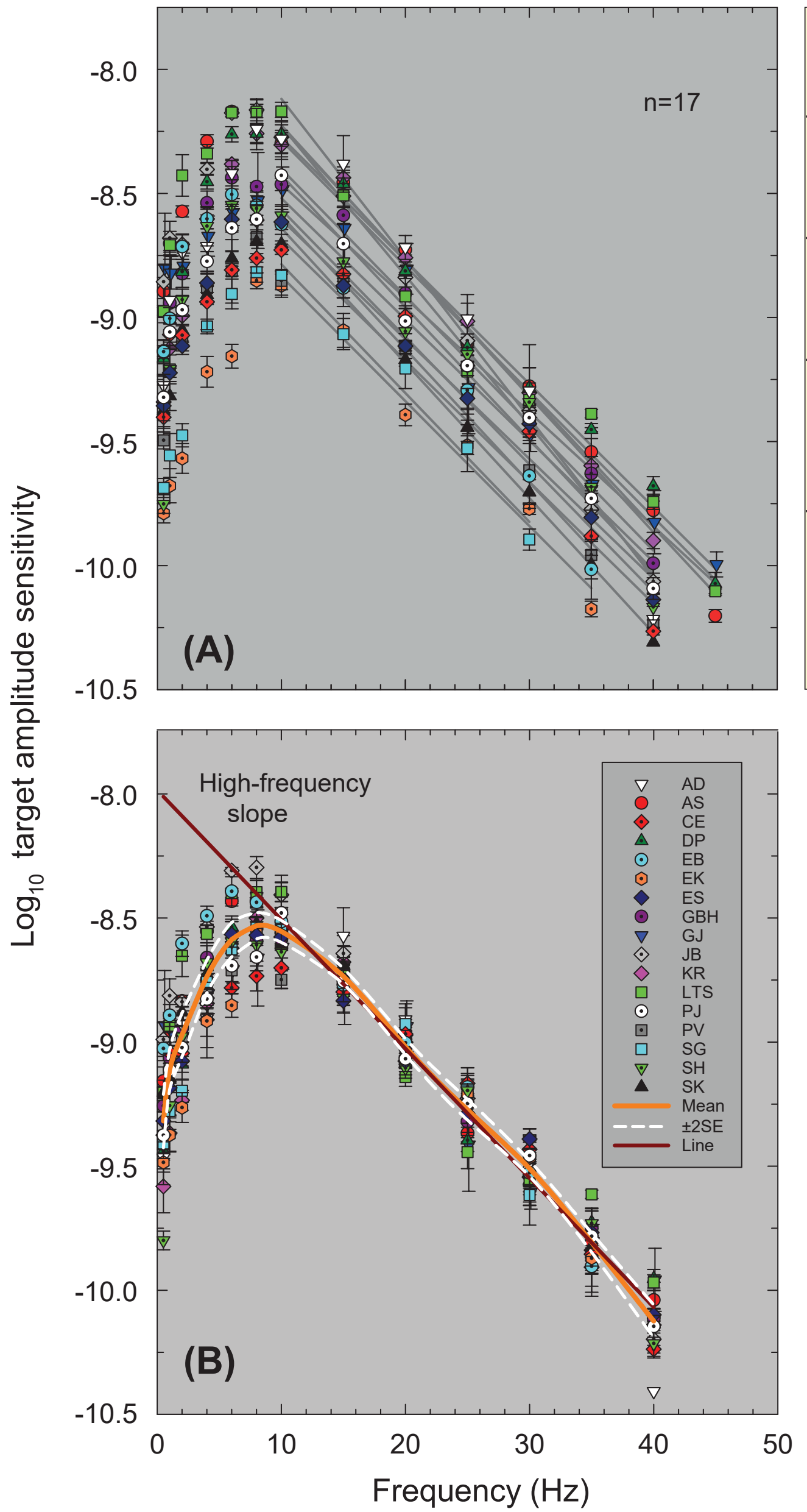

Flicker sensitivity

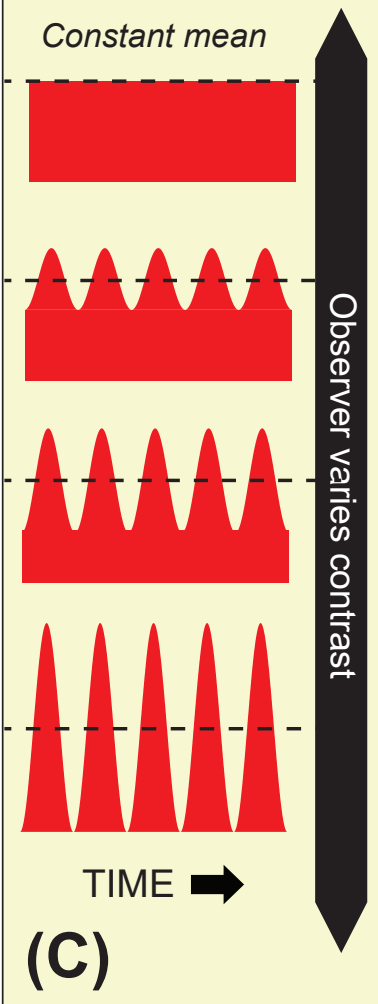


FIGURE 3

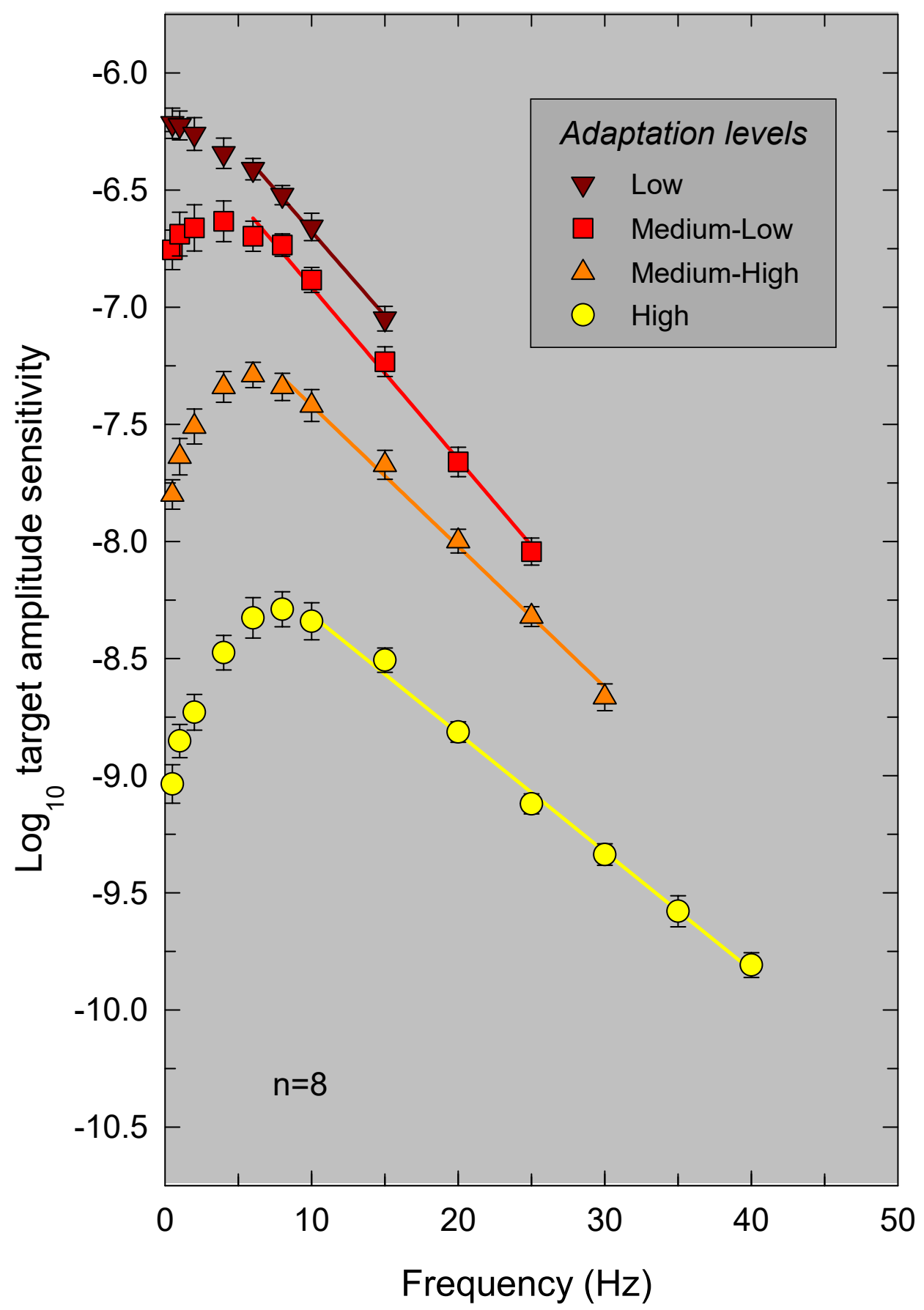


FIGURE 4

SEQUENTIAL MODEL

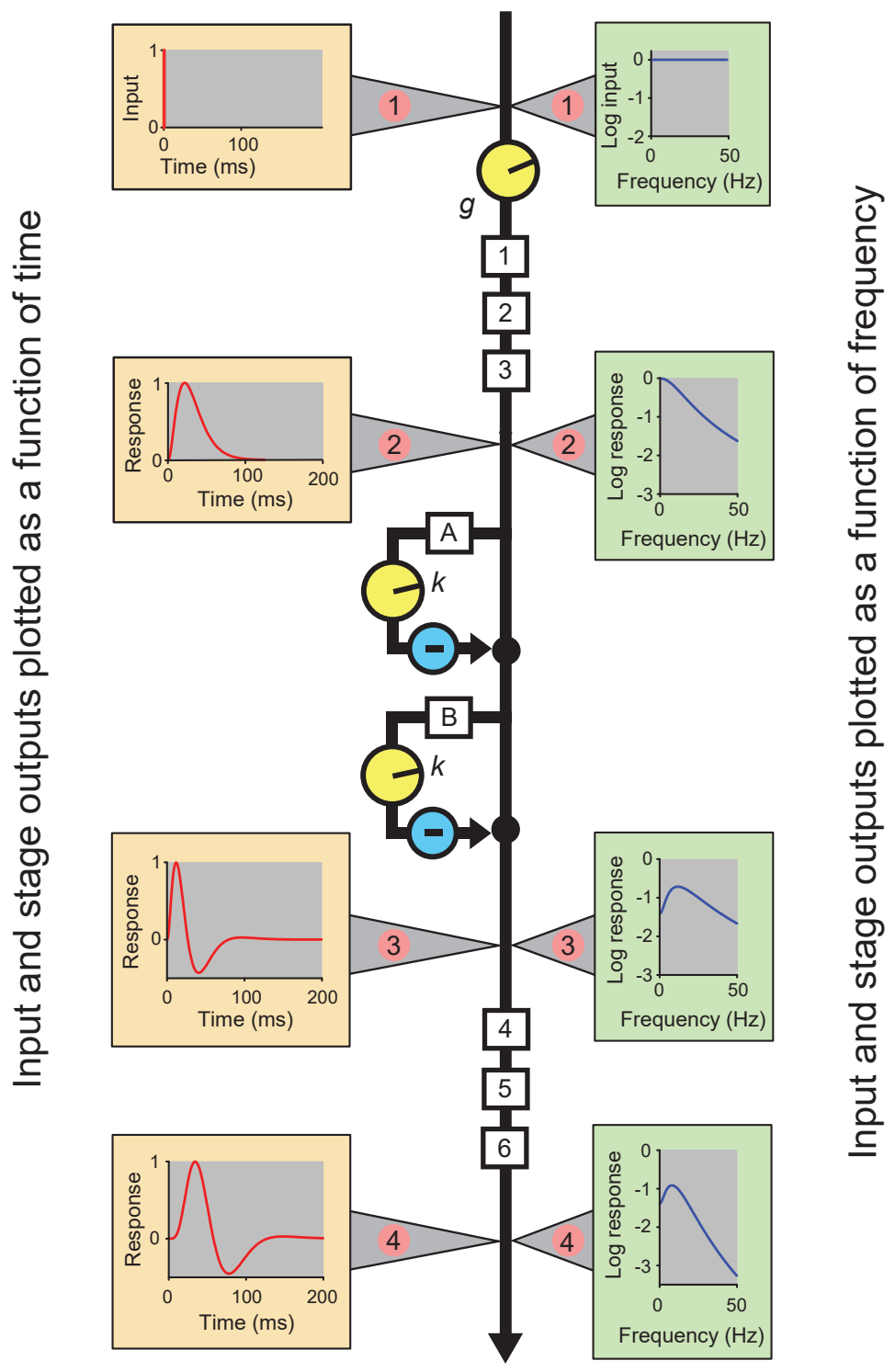


FIGURE 5

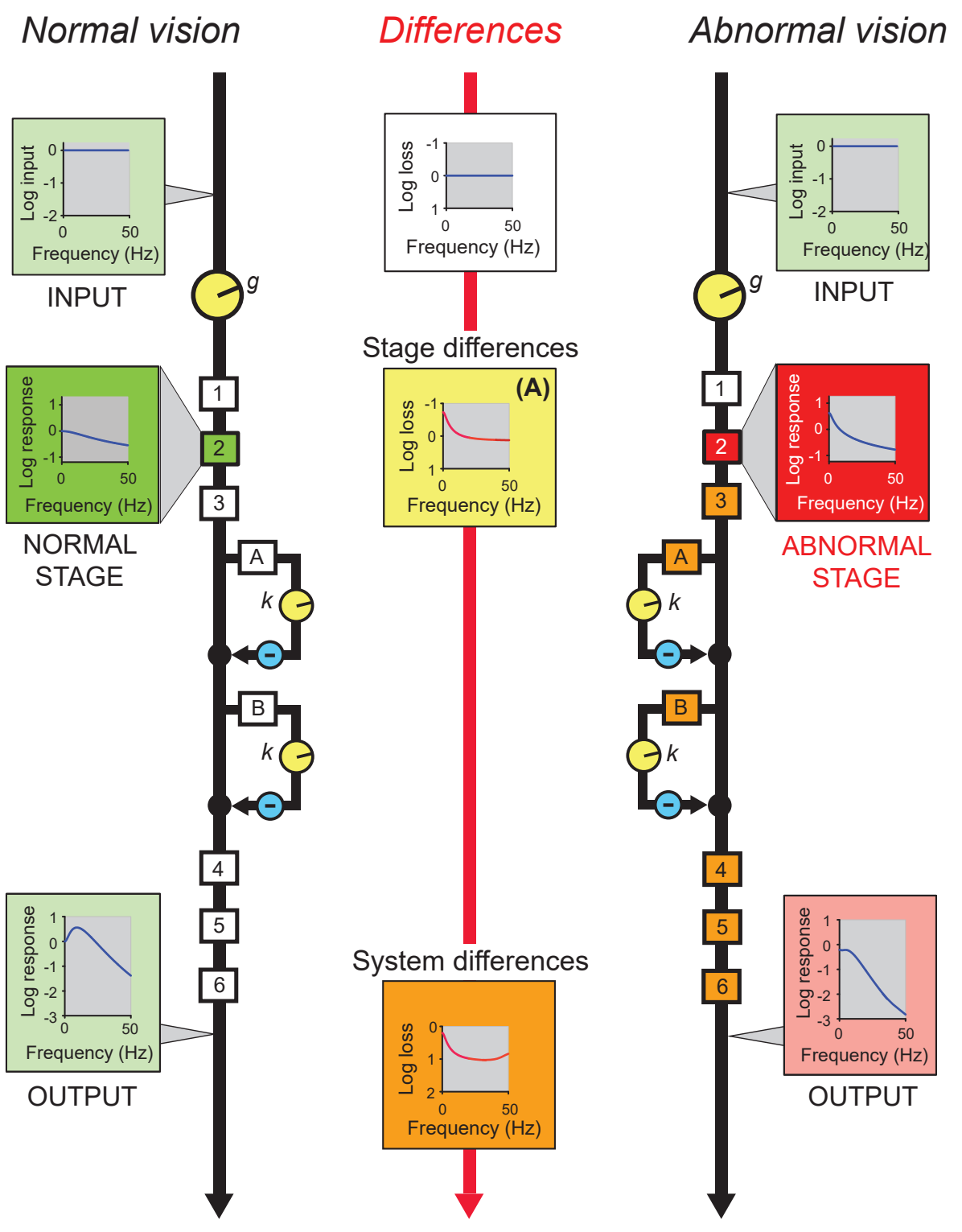

Examples of stage differences

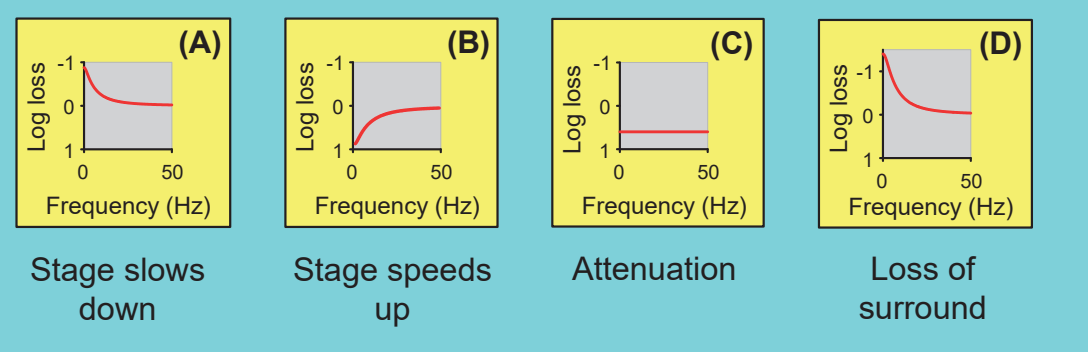



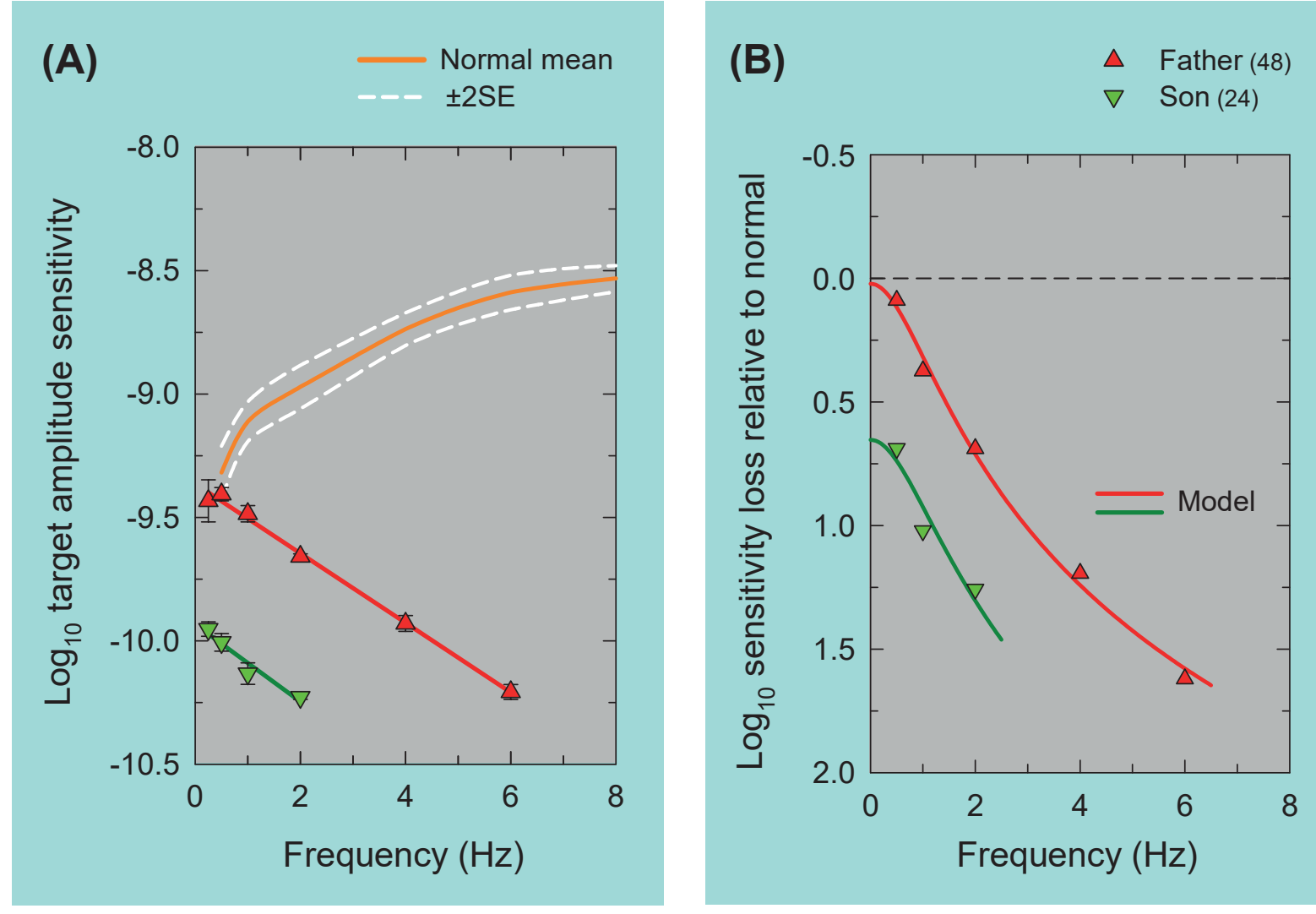
FIGURE 7
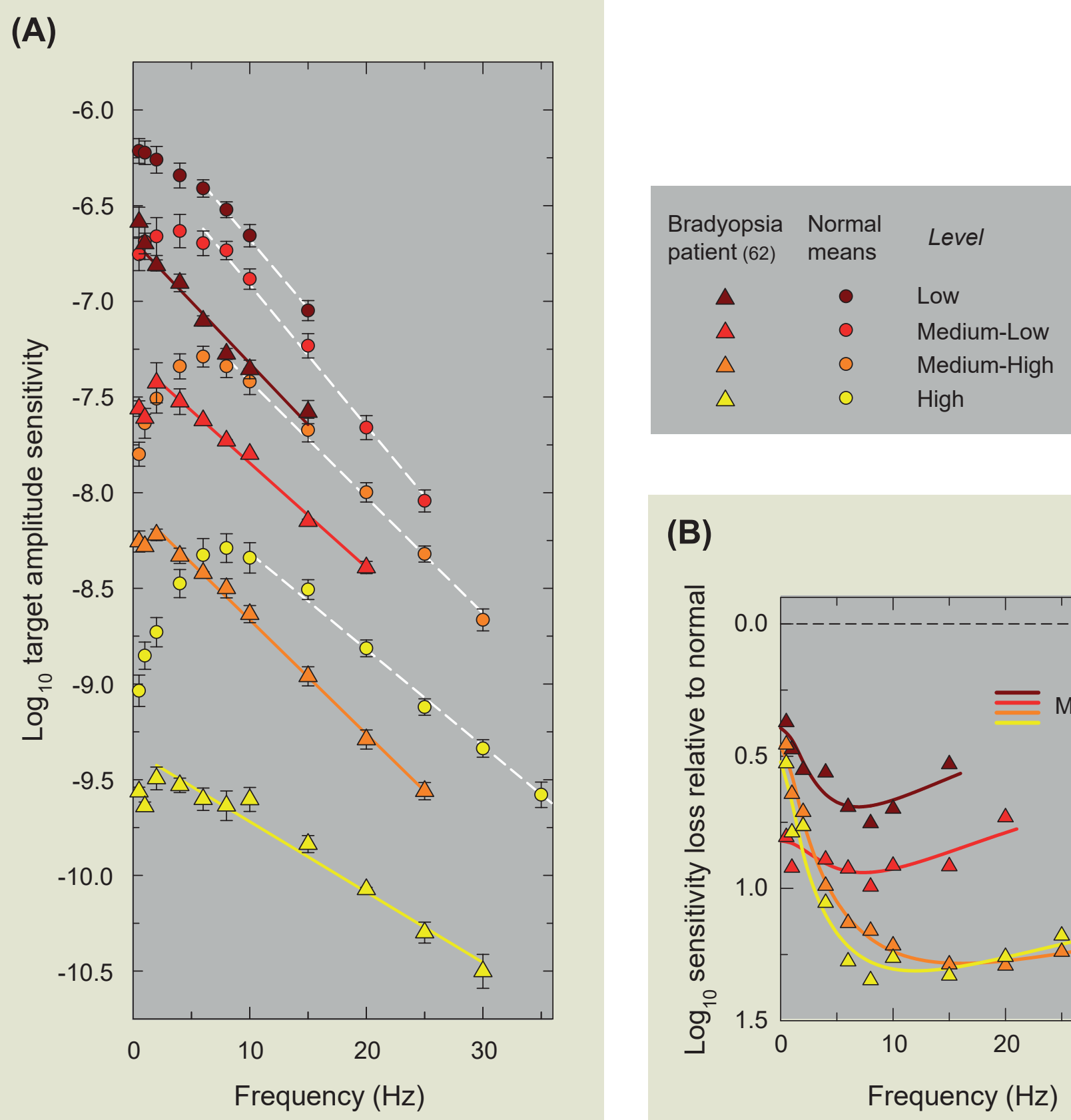

\section{(B)}

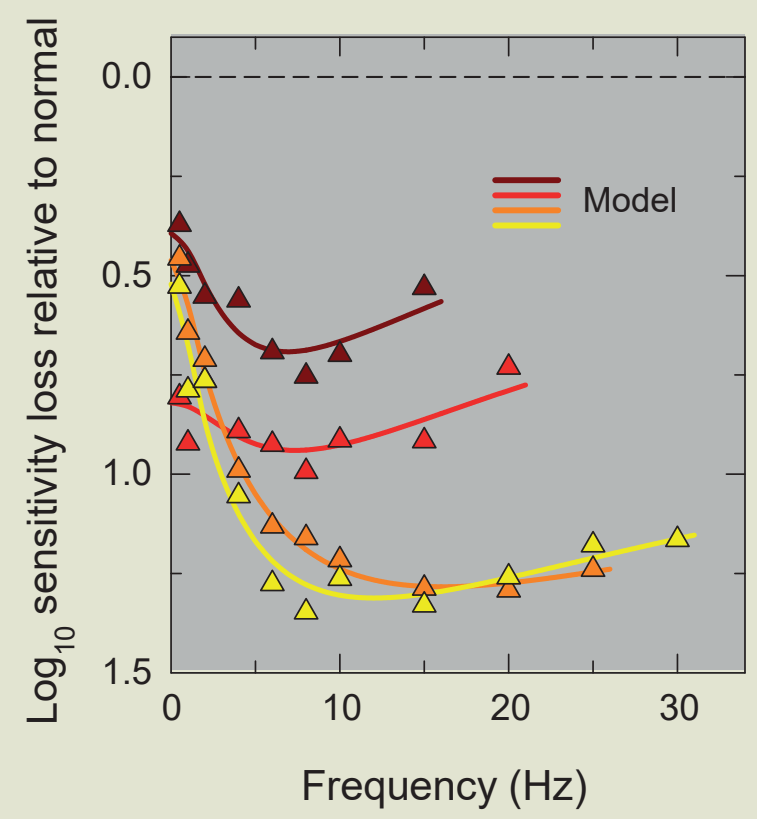



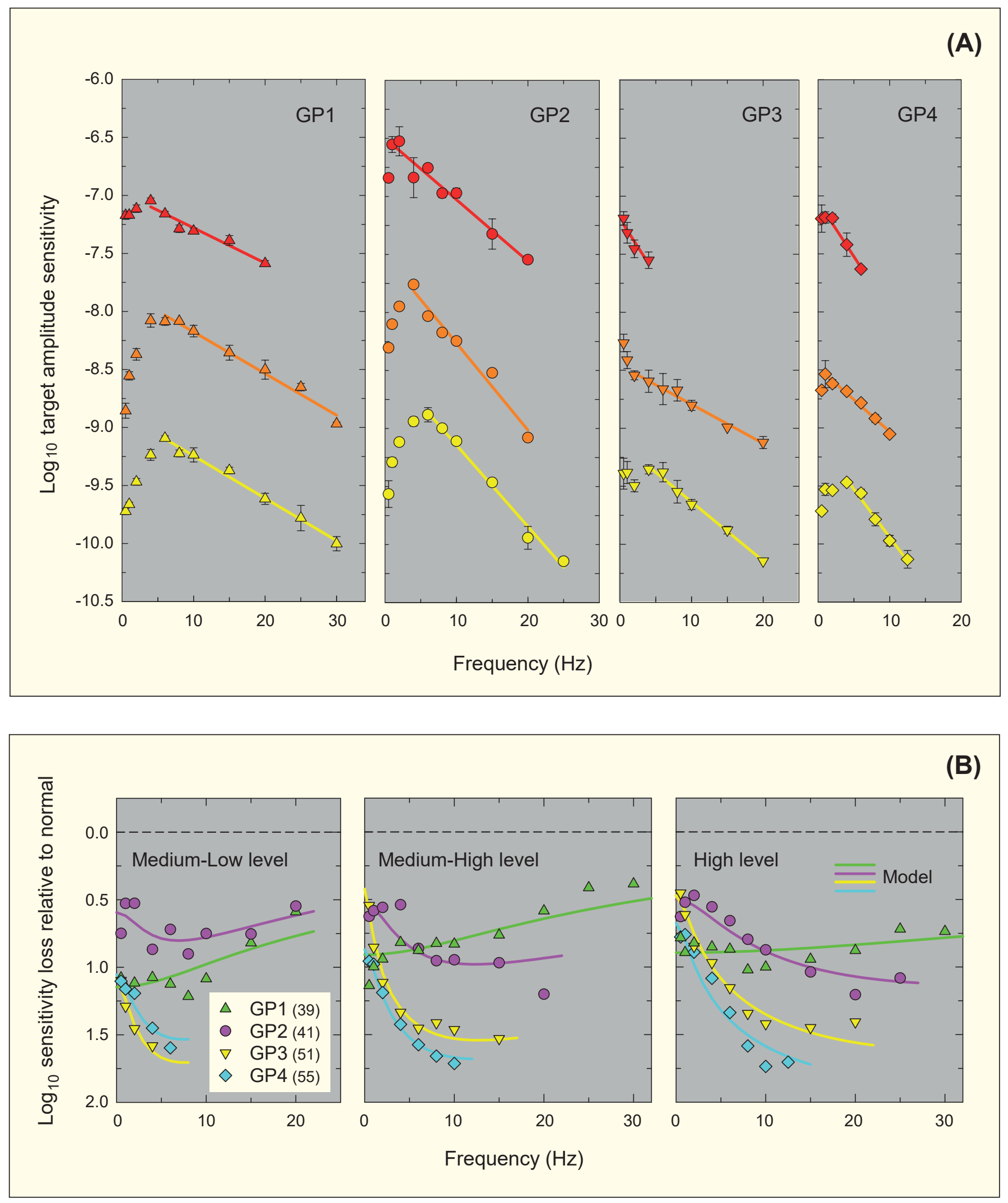
FIGURE 9
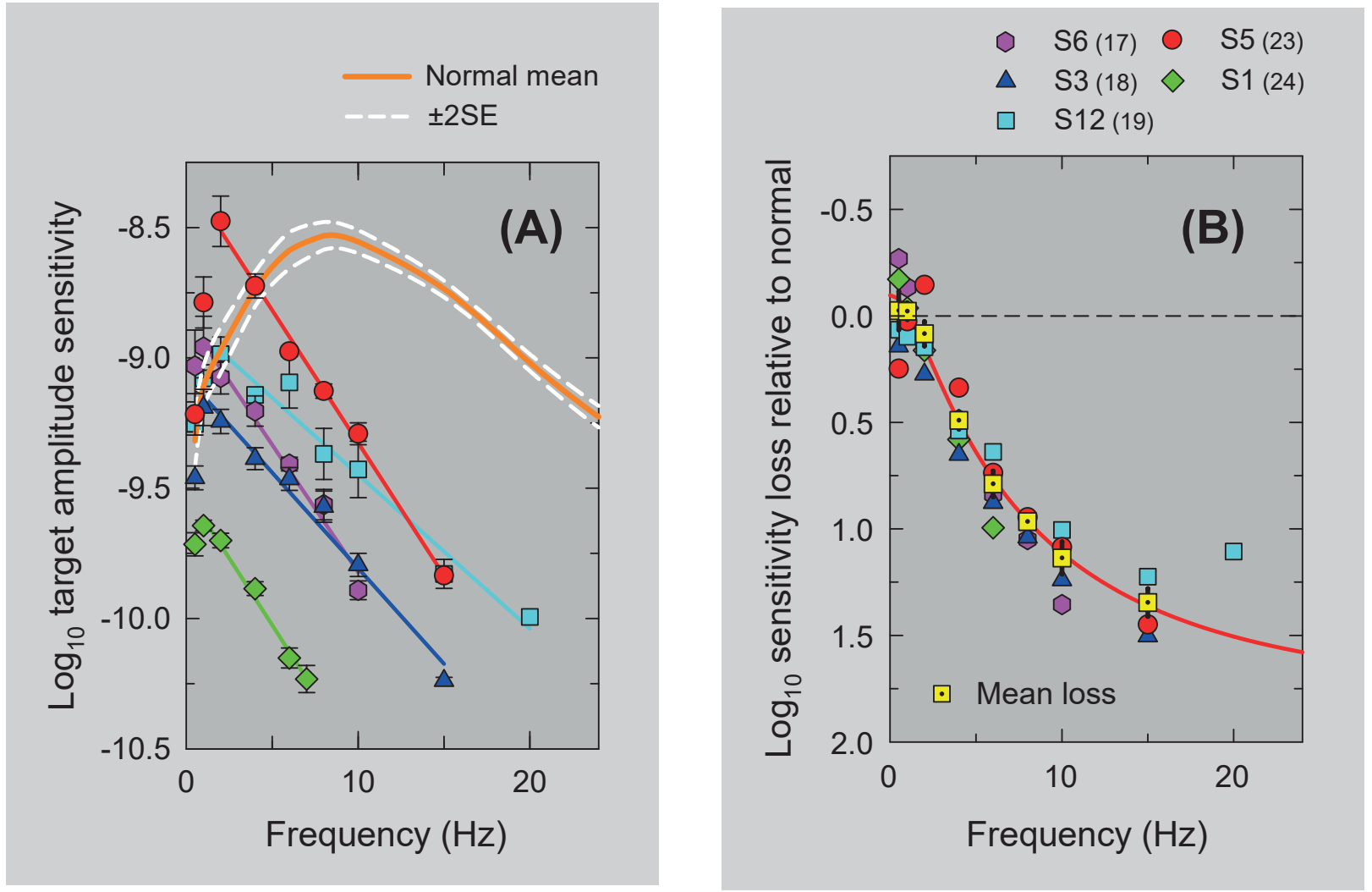

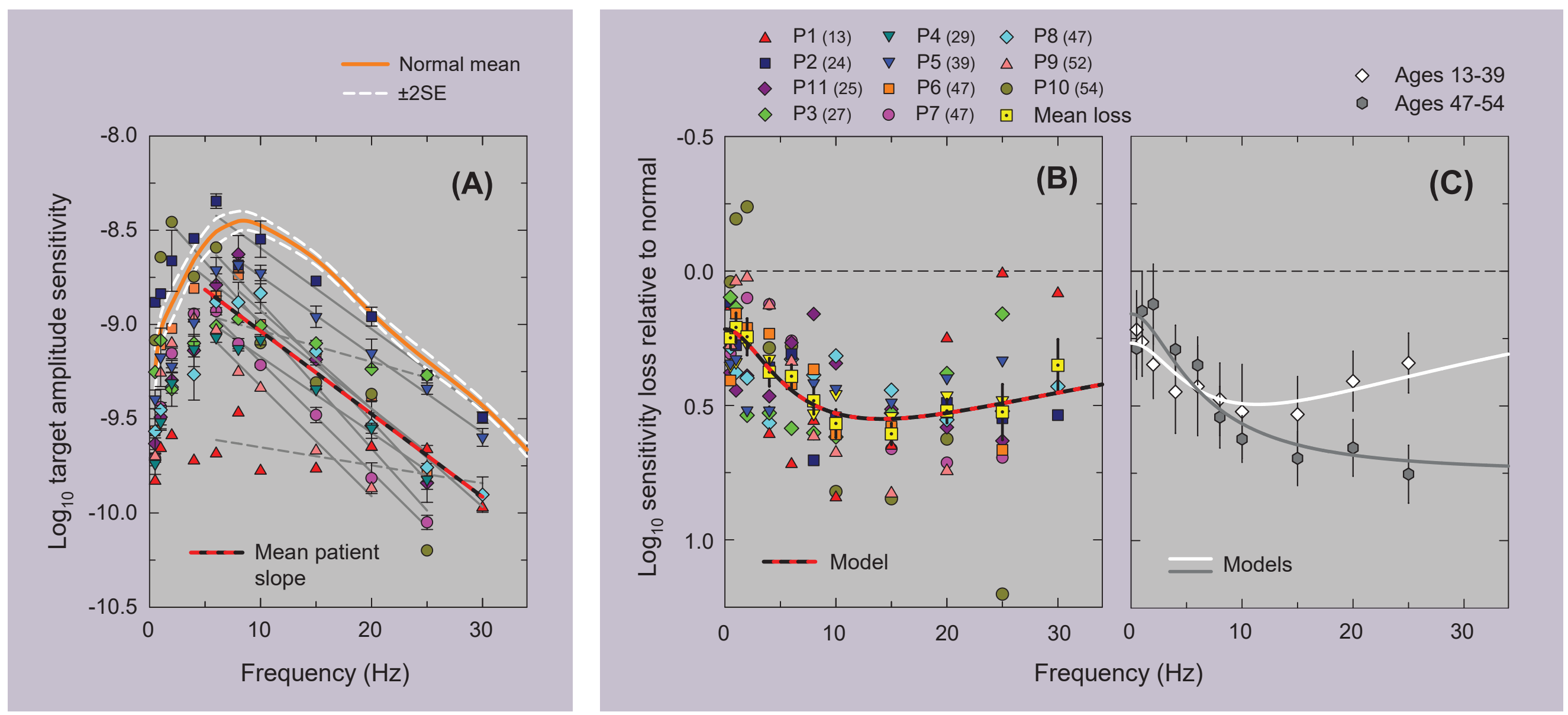
FIGURE 11
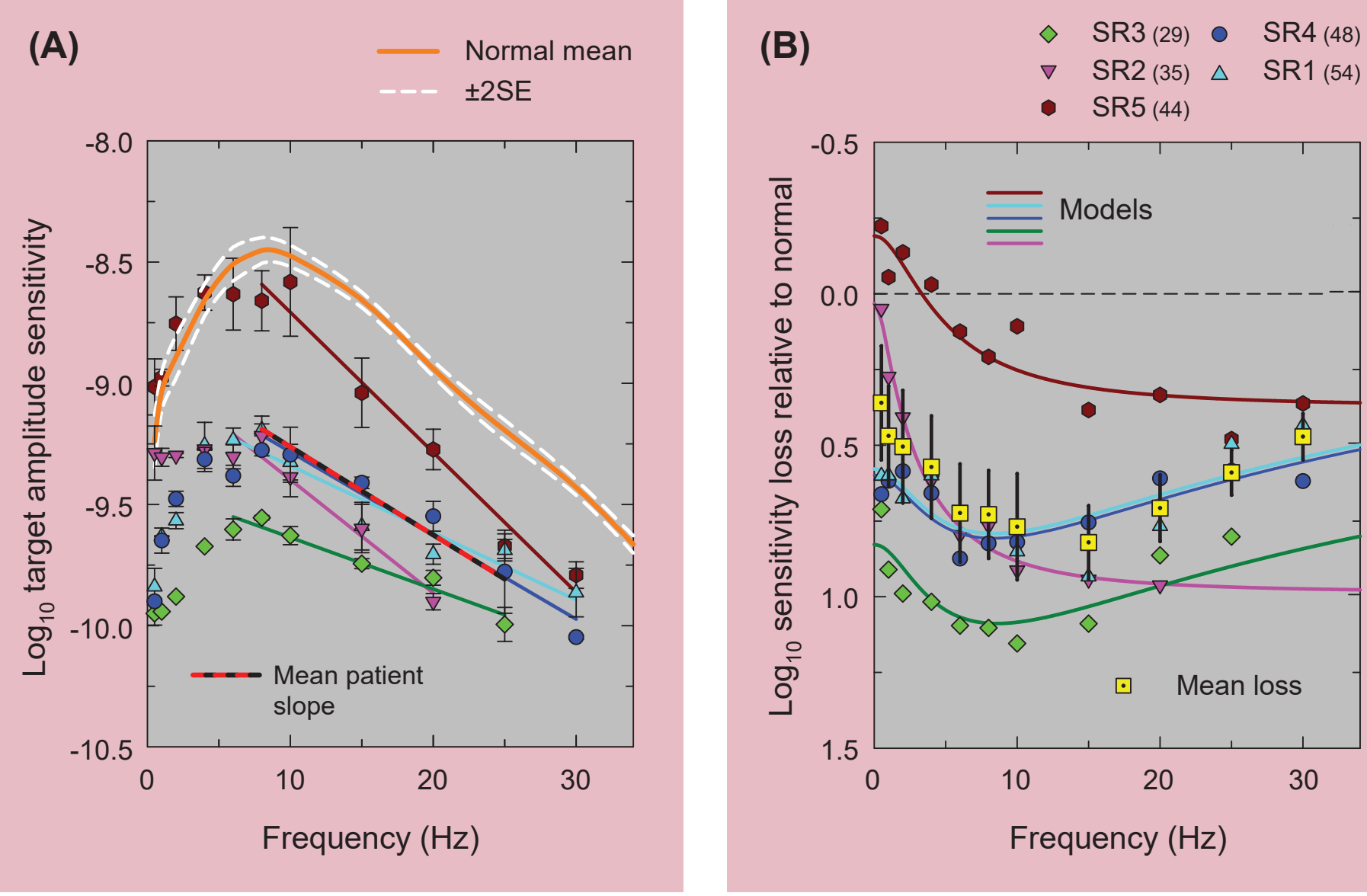
FIGURE 12

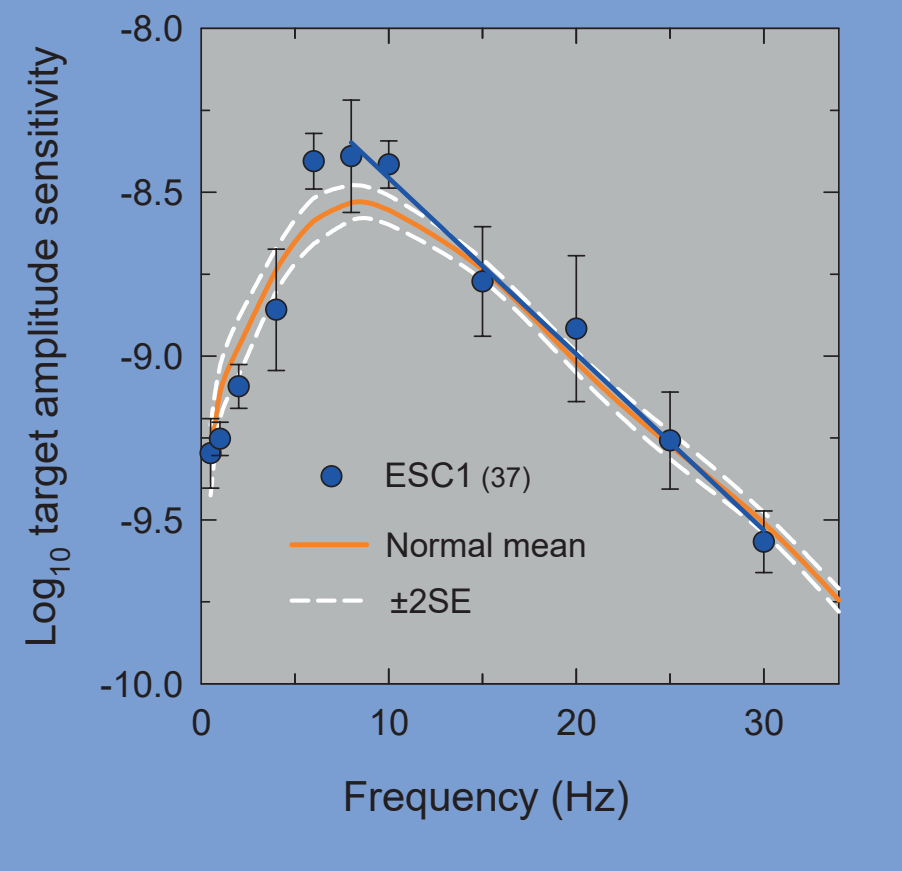


FIGURE 13

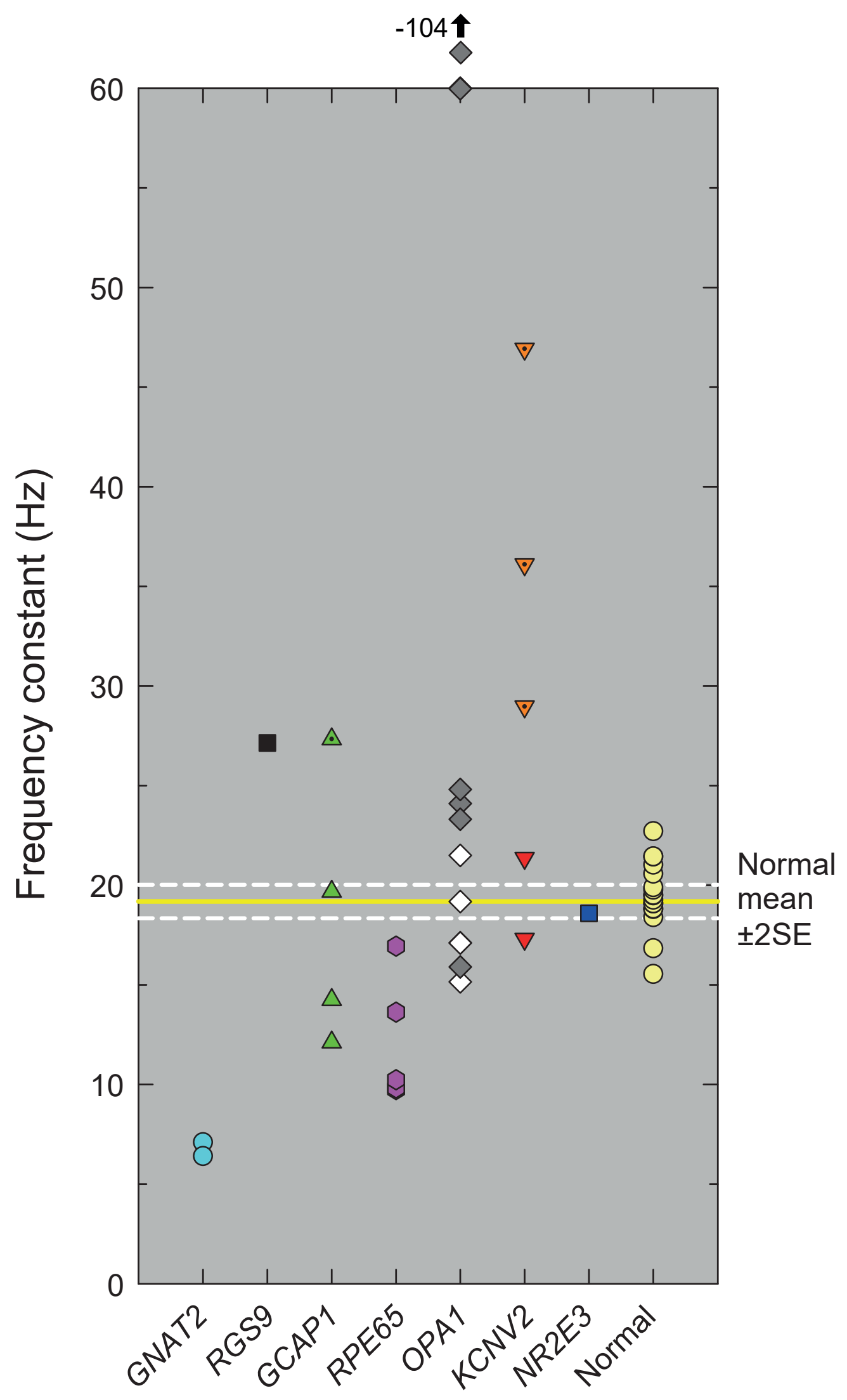



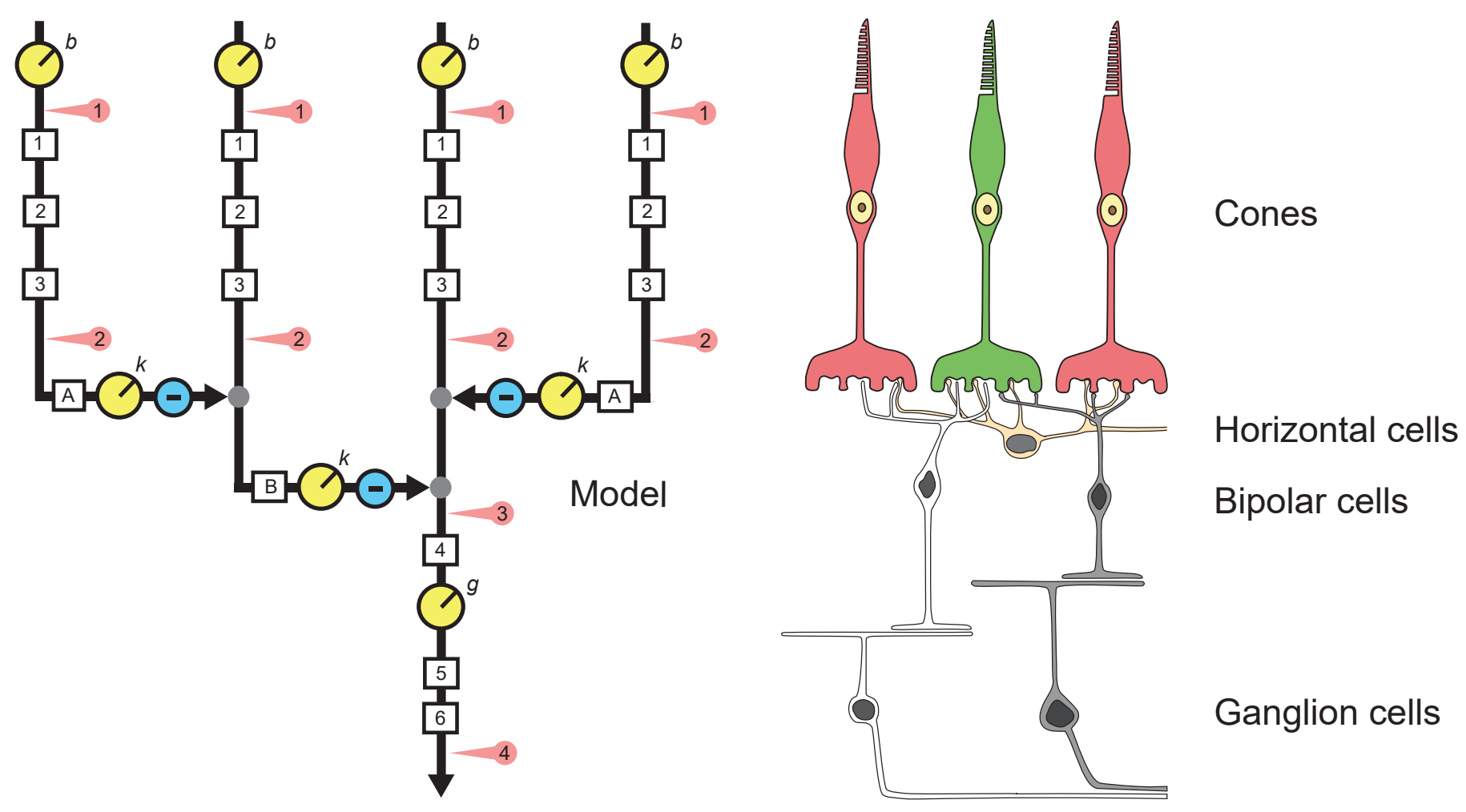


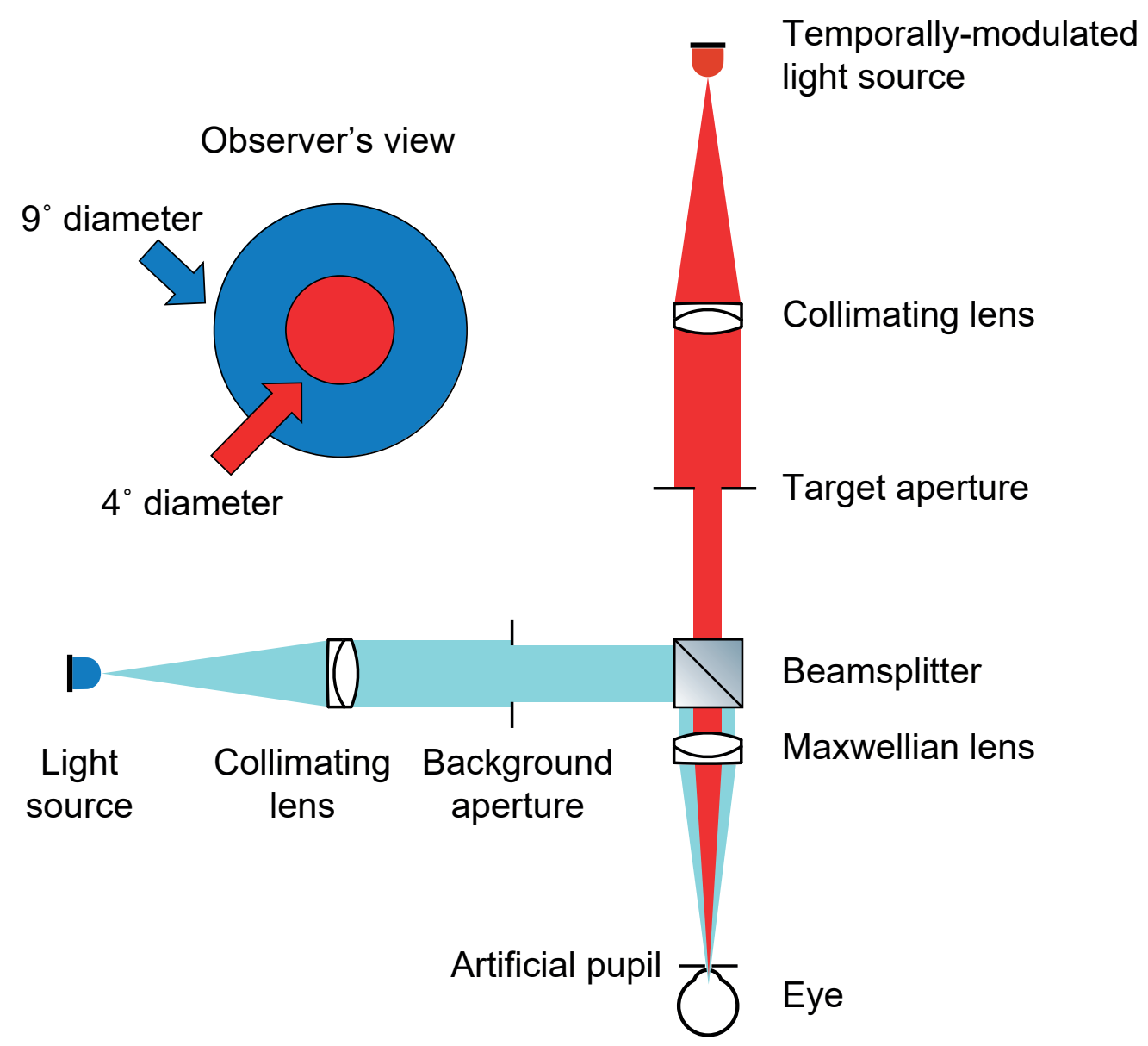

